\title{
Solar activity and its evolution across the corona: recent advances
}

Francesca Zuccarello ${ }^{1, *}$, Laura Balmaceda ${ }^{2}$, Gael Cessateur ${ }^{3}$, Hebe Cremades $^{4}$, Salvatore L. Guglielmino ${ }^{5}$, Jean Lilensten ${ }^{6}$, Thierry Dudok de Wit ${ }^{7}$, Matthieu Kretzschmar ${ }^{7}$, Fernando M. Lopez ${ }^{2}$, Marilena Mierla ${ }^{8,9,10}$, Susanna Parenti ${ }^{8}$, Jens Pomoell ${ }^{11}$, Paolo Romano ${ }^{5}$, Luciano Rodriguez ${ }^{8}$, Nandita Srivastava ${ }^{12}$, Rami Vainio ${ }^{11}$, Matt West $^{8}$, and Francesco P. Zuccarello ${ }^{13}$

1 Dipartimento di Fisica e Astronomia, Sez. Astrofisica, Università di Catania, Via S. Sofia 78, 95123 Catania, Italy *Corresponding author: francesca.zuccarel1o@oact.inaf.it

2 Instituto de Ciencias Astronómicas, de la Tierra y el Espacio, ICATE-CONICET, Av. España Sur 1512, J5402DSP, San Juan, Argentina

3 Physical-Meteorological Observatory/World Radiation Center, Davos, Switzerland

4 Universidad Tecnológica Nacional - Facultad Regional Mendoza/CONICET, Rodríguez 273, M5502AJE, Mendoza, Argentina

5 INAF - Osservatorio Astrofisico di Catania, Via S. Sofia 78, 95123, Catania, Italy

6 UJF-Grenoble 1/CNRS-INSU, Institut de Planétologie et d'Astrophysique de Grenoble (IPAG) UMR 5274, 38041 Grenoble, France

7 LPC2E/CNRS (UMR 7328) and University of Orléans, 3A avenue de la Recherche Scientifique, 45071 Orléans Cedex 2, France

8 Solar - Terrestrial Center of Excellence - SIDC, Royal Observatory of Belgium, Av. Circulaire 3, 1180 Brussels, Belgium

9 Institute of Geodynamics of the Romanian Academy, Jean-Louis Calderon 19-21, Bucharest-37 020032, Romania

10 Research Center for Atomic Physics and Astrophysics, Faculty of Physics, University of Bucharest, Str. Atomistilor 405, 077125 Magurele-Ilfov, Romania

11 Department of Physics, POB 64, 00014 University of Helsinki, Finland

12 Udaipur Solar Observatory, Physical Research laboratory, Udaipur 313001, India

13 Centre for mathematical Plasma Astrophysics, KU Leuven, Celestijnenlaan 200B, 3001 Leuven, Belgium

Received 16 June 2012 / Accepted 22 March 2013

\section{ABSTRACT}

Solar magnetism is responsible for the several active phenomena that occur in the solar atmosphere. The consequences of these phenomena on the solar-terrestrial environment and on Space Weather are nowadays clearly recognized, even if not yet fully understood. In order to shed light on the mechanisms that are at the basis of the Space Weather, it is necessary to investigate the sequence of phenomena starting in the solar atmosphere and developing across the outer layers of the Sun and along the path from the Sun to the Earth. This goal can be reached by a combined multi-disciplinary, multi-instrument, multi-wavelength study of these phenomena, starting with the very first manifestation of solar active region formation and evolution, followed by explosive phenomena (i.e., flares, erupting prominences, coronal mass ejections), and ending with the interaction of plasma magnetized clouds expelled from the Sun with the interplanetary magnetic field and medium. This wide field of research constitutes one of the main aims of COST Action ES0803: Developing Space Weather products and services in Europe. In particular, one of the tasks of this COST Action was to investigate the Progress in Scientific Understanding of Space Weather. In this paper we review the state of the art of our comprehension of some phenomena that, in the scenario outlined above, might have a role on Space Weather, focusing on the researches, thematic reviews, and main results obtained during the COST Action ES0803.

Key words. solar activity - active regions - coronal mass ejections - shock propagation - solar irradiance

\section{Introduction}

COST Action ES0803: Developing Space Weather products and services in Europe had the main objective of fostering collaboration between European Space Weather researchers, data providers, and service operators aiming at the assessment of the existing potential in operational Space Weather infrastructures and models, services, and collaborative research. The Memorandum of Understanding of this COST Action states that: "Space Weather originates mainly in solar activity and affects the interplanetary space and planetary magnetospheres, ionospheres, and atmospheres".

One of the main research tasks coordinated by this COST Action concerns the:

"Detailed identification of forefront scientific research on the physical processes responsible for various states of Space Weather: recent advances on solar dynamics (the origin of Space Weather), space plasma processes (the transmission process) and long-term variability of the Sun, interplanetary space and Geospace (the medium which moderates it) will be compiled and analyzed for a successful description, nowcasting and eventually forecasting of the state of the space environment".

The Scientific Work-plan, Methods, and Means of the COST Action foresaw the Identification of scientific understanding of physical processes which lead to various Space Weather phenomena. In particular, the authors of this paper were involved in the Task Sources of solar activity, which was divided in some sub-tasks, among which we recall the following ones: (a) Dynamics of active regions, flares, erupting 
prominences, CMEs, magnetic helicity transport; (b) Simulations of solar eruptions, particle acceleration, and turbulence; (c) three-dimensional (3D) reconstruction of CMEs from spacecraft observations.

We therefore describe here the state of the art of our knowledge on these phenomena, focusing on the main results on the Sources of solar activity obtained during this COST Action, and providing some illustrative examples of the researches that have been carried out thanks to this collaboration.

In this regard, we would like to stress that the rich and complex variety of phenomena observed in the solar atmosphere provides precious information that helps us improve our understanding of the modes of interaction between a plasma and magnetic fields of the order of $10-10^{3}$ Gauss. In particular, the study of active regions (ARs) on the Sun supplies information on how a plasma can be modified, in terms of temperature, density, velocity, etc., by the presence of magnetic fields that emerge from sub-photospheric layers as bundles of magnetic flux tubes, and how successively the magnetic field spreads over the plasma itself.

Nowadays, the different layers of the solar atmosphere are accessible with different spatial resolution. With current instruments it is in fact possible to achieve a spatial resolution of $\leq 0^{\prime \prime} \cdot 2$ in photosphere and chromosphere and $1^{\prime \prime}-2^{\prime \prime}$ in corona. This makes it very difficult to link the different observations and properly interpret them to give a global view: the fact that the phases of activation and eruption of a filament are followed in chromosphere and corona by different instruments, and the difficulty in identifying different strands and small features at different atmospheric heights, are typical examples of this problem.

Within the above-mentioned limitations, during the last few years the possibility of carrying on observations from both Earth and space in various spectral ranges has provided new and important indications on the modes of the interaction between the solar plasma and localized magnetic fields. However, if we take into account the evolution of an active region, that represents the typical expression of such interaction, in spite of the improvement in the observations and of the elaboration of more accurate and sophisticated models, we are not yet able to answer some fundamental questions. For example, why the emergence of a bundle of magnetic flux tubes does not give rise to ARs with the same type of evolution, or why the decay and diffusion of the magnetic field are so different from AR to AR.

On the whole, the emergence of magnetic flux involves regions with plasma $\beta$ (= ratio between plasma pressure $P_{\mathrm{g}}$ and magnetic pressure $B^{2} / 2 \mu$, with $B$ magnetic field strength and $\mu$ magnetic diffusivity) both $\gg 1$ and $\leq 1$, optical thick and optical thin layers, radiative phenomena observed at wavelengths ranging from radio to X-ray bands, through processes that possess an intrinsically three-dimensional nature.

Observations show that when the topology of the magnetic field of an AR becomes highly complex, the configuration may become unstable and an energy release process involving a flare and/or an eruptive prominence may occur, eventually causing the expulsion of magnetized plasma from the solar atmosphere, giving rise to coronal mass ejections (CMEs).

Flares are strong explosions that involve different layers of the solar atmosphere: they are due to a sudden release of energy $\left(10^{22}-10^{25} \mathrm{~J}\right)$ in areas previously characterized by variations in the magnetic field configuration (formation of current sheets, presence of null points, etc.) and energy budget. Flares have an evolution time varying from a ten of minutes to some hours. The phenomena that occur during a flare produce heating of the chromospheric and coronal plasma and intense electric fields that accelerate charged particles.

Coronal mass ejections are expulsions of magnetized plasma from the solar corona and, from an energetic point of view, they reach the same (or higher) order of magnitude of the flares; they can moreover pour in the space up to several billion tons of coronal plasma. Such phenomena can occur many times a day and can accelerate the solar plasma up to $3000 \mathrm{~km} \mathrm{~s}^{-1}$. Even if they have been extensively studied since their discovery, back in the 1970s of the XX century, a complete model for their explanation is still lacking.

Large-amplitude waves in the solar corona are a direct consequence of the dynamic magnetic activity of the corona: for instance, shock waves associated with eruptions occurring in the corona are believed to be linked to a number of transient phenomena such as metric type II bursts, Moreton and extreme ultraviolet (EUV) waves, and solar energetic particle (SEP) events. Eruptive phenomena, often caused by the sudden release of energy stored in the coronal magnetic field during processes of magnetic reconnection, can produce serious effects on the Sun-Earth environment and can trigger a series of phenomena (auroras, magnetic storms, charged particle reaching the ionosphere of the Earth), which are currently studied in the context of the Space Weather (see, e.g., Messerotti et al. 2009). In this regard, an improvement of our forecasting capabilities about the effects of solar phenomena on the Space Weather is essential in order to guarantee that ground- and satellite-based technologies work in a reliable way for a more innovative and safer society.

In the framework of the solar activity investigation, an important role is played by the Total Solar Irradiance (TSI). Understanding the variability of the TSI and of the solar spectral irradiance is important for assessing the solar radiative forcing of the terrestrial atmosphere. For Space Weather applications, the impact of the UV band on the ionospherethermosphere system is a major issue. This variability may also eventually affect climate on long-term scales.

All these topics, and the recent advances obtained during the last years, will be described in this review. As stated above, a particular emphasis will be given to the results obtained by the authors during the last 3 years in a Working Group devoted to the Progress of scientific understanding of Space Weather, in the framework of the COST Action ES0803.

In Section 2 we describe the main observational evidence related to active region formation, evolution, and decay; Section 3 describes the physical properties of ARs in the corona, taking into account the plasma temperature, density, and velocity parameters; in Section 4 the role of the magnetic helicity and its variation in time and space is stressed, in relation to the occurrence of erupting phenomena; Section 5 is devoted to the description of the physical properties of eruptive prominences; Section 6 reviews the kinematics of eruptive prominences and associated CMEs; in Section 7 we report on the main CME models developed so far, taking into account the difference between models requiring magnetic reconnection and those that do not; Section 8 describes the main 3D reconstruction techniques used to determine the properties of CMEs, as well as three case studies which present events observed when the two twin STEREO satellites were at different positions with respect to the Earth (and to the SOHO satellite); in Section 9 we report the complete description of the evolution 

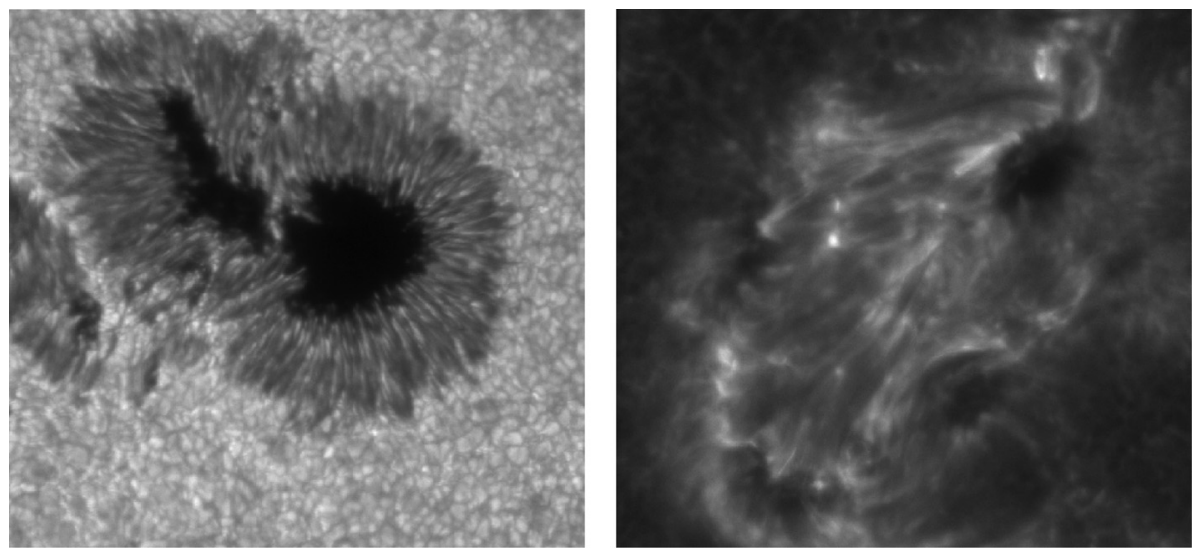

Fig. 1. Left panel: sunspot belonging to NOAA 11263, observed in the G band on August 6, 2011. Right panel: AFS observed above an EFR in the chromosphere (Ca II H line). The images were acquired by the Swedish 1-m Solar Telescope (SST).

of two CME events from their source region in the solar atmosphere to the Earth; in Section 10 the coronal propagation of shocks and the acceleration of energetic particles are discussed, both from the observational and the modeling points of view; the most recent results on the solar irradiance variability are reviewed in Section 11, and finally Section 12 is devoted to the summary and the conclusions.

\section{Active region formation, evolution, and decay}

This section provides an overview of the different evolutionary phases of the main Sources of solar activity: the active regions.

ARs are the most conspicuous manifestation of the interaction between magnetic field and plasma in the solar atmosphere. During the last two decades, investigations carried out using high-resolutions observations and numerical simulations greatly improved our understanding about the physical processes behind ARs appearance into the solar atmosphere. It is now well agreed that magnetic flux tubes - rooted in the toroidal field generated deep in the solar interior by the $\alpha-\Omega$ dynamo mechanism (e.g., Charbonneau 2010) - cross the convection zone via magnetic buoyancy on a time scale of $\sim 2$ months and finally emerge into the photosphere and the upper atmospheric layers (e.g., Fan 2009), where their interaction with the atmospheric plasma gives rise to the activity phenomena observed in these layers.

Magnetic flux emergence is a process involving a wide range of spatial scales: from the large and complex ARs present during solar maxima - with magnetic flux up to $\sim 10^{23} \mathrm{Mx}$ $\left(1 \mathrm{Mx}=10^{-8} \mathrm{~Wb}\right)-$ that often host the most violent phenomena of energy release (flares, eruptive prominences, CMEs), to small bipolar flux concentrations - ephemeral regions and granular bipoles, with fluxes from $10^{16}$ to $10^{19} \mathrm{Mx}$ (Hagenaar 2001; Martínez González \& Bellot Rubio 2009) - that populate the quiet Sun at any time of the solar cycle. We generally refer to both ARs and smaller flux patches as emerging flux regions (EFRs).

\subsection{Formation}

From an observational point of view, the most prominent phenomenon that occurs in the photosphere during the formation of ARs is the appearance of sunspots (see, e.g., Solanki 2003). They are characterized by a dark region (umbra) surrounded by a (generally) radial structure (penumbra), as shown in Figure 1 (left panel). However, all the atmospheric layers are involved by flux emergence: after ARs are observed in the photosphere, faculae are observed in the chromosphere, and bright regions and loops in the hotter coronal layers. Also smaller EFRs can give rise to some disturbances of the overlying layers, especially in the low chromosphere.

Nowadays, the SOlar and Heliospheric Observatory (SOHO) and Hinode satellites, balloon- (SunRISE), and ground-based telescopes (THEMIS, VTT, DST, SST) provide spectroscopic and spectro-polarimetric observations with high spatial (down to $0 ! 2$ in photosphere, $1^{\prime \prime}-2^{\prime \prime}$ with UV-EUV SOHO/SUMER and Hinode/EIS) and temporal (0.5-1 s) resolution. The analysis of simultaneous measurements of the vector magnetic field and of the thermodynamical properties of the plasma allows us to reconstruct a clear picture of the EFRs dynamics in the lower atmospheric layers during their formation (see, e.g., van Driel-Gesztelyi 2002):

- flux concentrations do not simply appear as pairs of opposite polarities: they are formed by several emergence events with mixed polarities (sea-serpent structure) that have a net polarity clearly distinguishable, as they separate from each other;

- in the $G$ band (4035 $\AA$ ) and in the continua of photospheric lines, the granulation in the EFRs' sites appears disturbed, with the formation of anomalous big granules and dark lanes aligned along the axes joining opposite net polarities;

- fibrils connect the opposite polarities of the EFRs in the low chromosphere, as shown in $\mathrm{Ca}$ II observations in the near-infrared and in the near-ultraviolet;

- arch filament systems (AFS), connecting the opposite polarities of the ARs, are formed in the chromosphere (see Fig. 1, right panel): they have upward motions at their top of $2-3 \mathrm{~km} \mathrm{~s}^{-1}$ and downflows along their edges of about $10 \mathrm{~km} \mathrm{~s}^{-1}$, both slowing with time (Spadaro et al. 2004; Zuccarello et al. 2005).

The cross-sections of the rising magnetic flux tubes with the photosphere, which later form the main polarities of EFRs and correspond to sunspots in the case of an emerging AR, have the emerging magnetic field nearly vertical (with respect to the solar surface), whereas it is more horizontal in the region in between them. The emerging loops exhibit downward motions along their legs, i.e., vertical fields have downflows, while flux 
regions with nearly horizontal inclination show upflows during the emergence phase (Zuccarello et al. 2009b). Usually the preceding sunspot ( $p$-spot, i.e., the sunspot that precedes in the solar rotation) appears first, followed after few hours/days, by the following sunspot (f-spot, i.e., the eastern spot or the sunspot that follows in the solar rotation): the $p$-spot is generally larger, more compact, and with a larger magnetic flux than the $f$-spot (see, e.g., Solanki 2003; Fan 2009 and references therein).

Large ARs have typical lifetime of the order of $\sim 1-2$ months and magnetic field intensity of about 2000-2500 G for large, well-developed sunspots umbrae, and 1000-1500 G for sunspots penumbrae, where the field lines radially oriented around the umbra are also more inclined. Magnetograms show that the magnetic field extends well beyond the photospheric structures, embedded in facular regions with field strength of $\sim 1000 \mathrm{G}$. The magnetic field piles up at the border of large convective granulation cells (supergranules, e.g., Del Moro et al. 2007). At smaller scales, the magnetic field strength is of $\sim 1500 \mathrm{G}$ in pores, dark structures visible in the photosphere as large as a ten of granules, and falls down to about 800 $1000 \mathrm{G}$ for the footpoints of the smaller ephemeral regions, and down to $400-500 \mathrm{G}$ for the tiny polarities of the granular bipoles, in equipartition with the photospheric granulation.

The azimuth of the magnetic field vector sometimes deviates from the straight positive-negative connection between the polarities, indicating the presence of some twist and/or writhe in the emerging structures (Holder et al. 2004). This is particularly evident in the case of complex ARs, like sunspots group with $\delta$ configuration (Tian et al. 2005), while it is yet uncertain for the smaller bipoles (Guglielmino et al. 2012).

In this context, it is worthwhile to mention that the possibility to determine, since the very first phase of its appearance in the solar atmosphere, whether or not the buoyancy of a bunch of flux tubes might give (or not) rise to a well-developed AR is essential in order to predict the level of solar activity for the following days/weeks, with important consequences on Space Weather forecasting. This was investigated for young ARs by Spadaro et al. (2004) and Zuccarello et al. (2005). They analyzed the differences found in AFS plasma motions in recurrent and short-lived ARs, respectively.

\subsection{Evolution}

The evolution of the EFRs strongly depends on the environment that the emerging flux encounters in the site of emergence. In the atmospheric layers that hosted a previous emergence event, whose flux has not yet spread over, interactions with the new flux may occur, giving rise to transient phenomena, like brightenings and jets (see, e.g., Guglielmino 2012 and references therein).

Numerical models predict that different flux systems pushed against each other may undergo magnetic reconnection, so that this process is believed to play a crucial role in the dynamics of the interactions (Yokoyama \& Shibata 1995; Archontis et al. 2005). The geometry of the interacting flux systems is a fundamental factor, in particular the relative orientation between the emerging flux system and the pre-existing magnetic loops. When they are nearly parallel, the interaction is weak, while it gives rise to strong energy release when they are nearly antiparallel (Galsgaard et al. 2007; MacTaggart 2011).

Observational evidence supports these findings, showing the response of the chromosphere and of the corona to the emerging photospheric field. Typical signatures of such processes are:

- presence of brightenings co-spatial with the contact region of the EFRs with elements of the pre-existing flux in various chromospheric and coronal lines (Guglielmino et al. 2010);

- occurrence of microflares in X-rays (Kubo et al. 2003) and even small flares in AFSs sites, when a new AFS rises from the lower layers (Zuccarello et al. 2008);

- rearrangement of the topology of the coronal arcades connecting the old and the new flux systems;

- ejections of plasma both at chromospheric level $(\mathrm{H} \alpha$ and $\mathrm{H} \beta$ surges) and at coronal heights (X-ray jets) with a characteristic inverted-Y shape, triggered by both large-scale EFRs (Moreno-Insertis et al. 2008) and smaller scale emergence events (Guglielmino et al. 2010).

Plasma motions during ejection episodes achieve speeds of about $50-100 \mathrm{~km} \mathrm{~s}^{-1}$ and last for about half an hour. Sometimes they are recurrent, when emergence events are repeated in time. The Doppler analysis of EUV lines shows upflows, with emission enhancements stronger in the lower layers and non-thermal broadening (Guglielmino et al. 2010). The brightenings may extend and involve the whole arcades connecting the opposite polarities of an AR, as we display in Figure 2 (left panel).

When the interaction occurs in a catastrophic way, the sudden energy release may trigger the onset of flares and of other eruptive phenomena, whose occurrence is of paramount importance for Space Weather conditions.

\subsection{Decay}

The decay of flux concentrations is still the less understood phase of flux dynamics in the solar atmosphere. Several authors have tried to summarize the main characteristics of this phase, but it is quite difficult to provide a general scenario able to include all the observations. The physical processes during this phase act essentially on small spatial scales. In addition to the possible cancellation due to magnetic reconnection with preexisting flux elements and to the subsequent partial annihilation between opposite polarities, there are essentially two ways to remove the flux: diffusion and in situ decay (van Driel-Gesztelyi 2002).

High-resolution spectro-polarimetric data recently provided by the new generation instruments, have indicated the key role that small magnetic elements, called moving magnetic features (MMFs), can have in the process of magnetic field decay and diffusion. MMFs are magnetic elements spreading away from sunspots. They are usually interpreted as the extensions of penumbral filaments that cross and emerge again into the solar surface. However, they are observed also in pores and sunspots without photometric penumbrae (Zuccarello et al. 2009a; Criscuoli et al. 2012), though inclined magnetic fields may be present around (Sainz Dalda et al. 2012).

MMFs are thought to carry flux away from ARs, which would cancel during the coalescence with flux elements of opposite polarities present in the facular regions, or would be recycled by large-scale flows. Another form of flux erosion is suggested by the observation of light bridges which form during the decay phase of the sunspots (see, for instance the light bridge in the top-left sunspot shown in Fig. 2, right panel). 

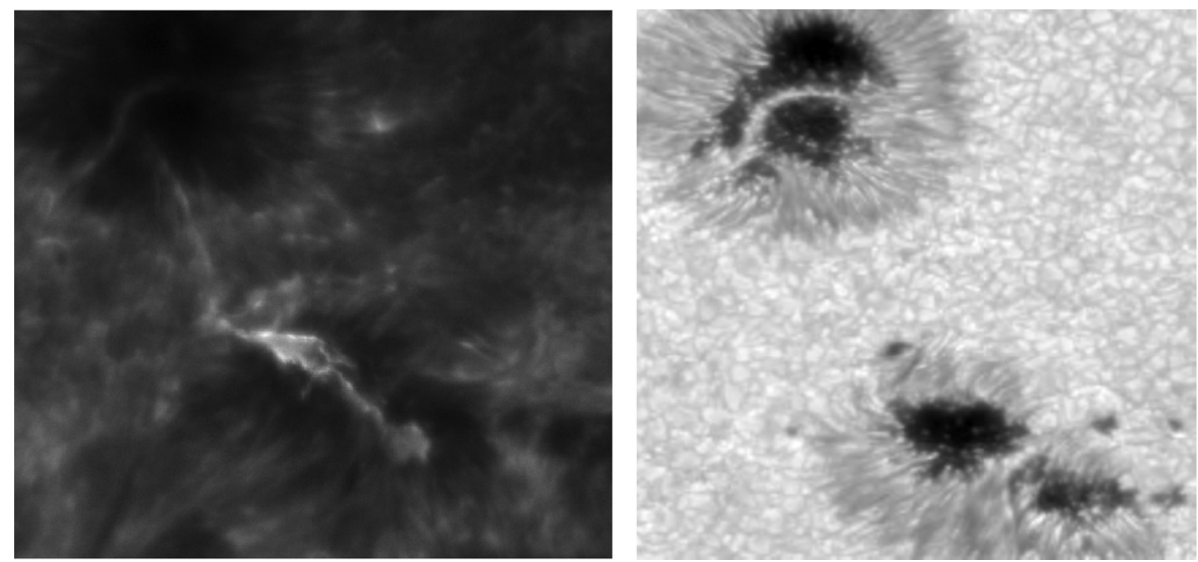

Fig. 2. Left panel: brightening connecting sunspots with opposite polarities in an AR, observed in the chromosphere in the core of Ca II $\mathrm{H}$ line. Right panel: sunspot group observed in the $G$ band during its decay phase; the sunspot on the left-top clearly shows a light bridge. Both images were taken by the SST on August 18, 2011.
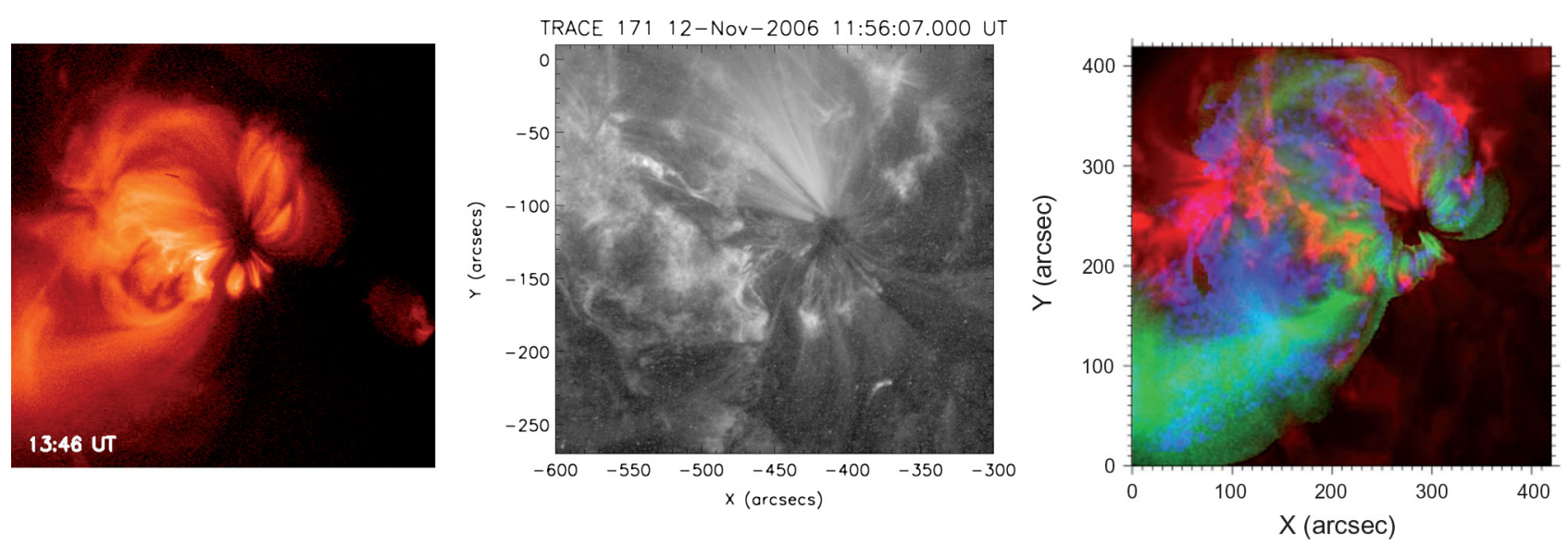

Fig. 3. Left: Hinode/XRT image of AR 10923 observed on November 12, 2006. Center: TRACE $171 \AA$ image of AR 10923 observed on the same day. Right: composite temperature maps of AR 10923. Green: thermal map at about 3 MK. Blue: thermal map at more than 5 MK. Red: Trace 171 Å intensity map. From Reale et al. (2009).

On smaller scale, the exponential decay observed in the remnants of granular bipoles suggests the existence of some mechanism that acts in situ by removing the flux from the solar atmosphere, analogously to turbulent diffusion (Guglielmino et al. 2012).

But the progress in this subject is expected to grow with observations at higher spatial resolution, hopefully provided in the next future by the European Solar Telescope (EST), the Advanced Technology Solar Telescope (ATST), and Solar Orbiter satellite. These instruments would provide new clues about the flux dynamics at small scale $(\sim 100 \mathrm{~km})$.

\section{Physical properties of Active Regions in the corona}

This Section provides an overview of the main observational properties of Active Regions.

The coronal counterpart of photospheric magnetic field concentrations giving rise to ARs is best visible in EUV and soft X-ray images where they appear as magnetic loops filled by dense plasma.

Soft X-rays image (e.g., Fig. 3, left panel) the AR core, which is the hottest part of the structure ( $3 \mathrm{MK}$ on average,
Reale 2010). This is made of short, low-lying compact loops with a density in the range $10^{8.5-11} \mathrm{~cm}^{-3}$ (e.g., Vaiana \& Rosner 1978; Ko et al. 2009). The density of these loops is lower than that of equivalent loops in steady state (Klimchuk 2006). In non-flaring conditions, the emission of these loops is very stable in time (e.g., $20 \%$ variation within hours) and suggests a filling factor between $3 \times 10^{-3}$ and 0.3 (e.g., Dere 2008, 2009).

EUV image mostly the AR periphery, as shown by Figure 3 (center panel). This is composed by "warmer" average $1 \mathrm{MK}$ loops, which are longer, less dense $\left(10^{8.5}<N_{e}<10^{10} \mathrm{~cm}^{-3}\right.$, Doschek et al. 2007), and less compact than those forming the core. Their density, as derived from observations, is above the values expected for a steady-state loop and their filling factor is estimated to be about 1 (e.g., Del Zanna \& Mason 2003; Warren et al. 2008). These loops sometimes connect adjacent ARs or/and may be generally visible in the AR core, overlying the X-ray loops.

EUV loops appear sometimes to be co-spatial with preexisting soft X-ray loops, suggesting a heating-cooling cycle (Ugarte-Urra et al. 2006, 2009; Cirtain et al. 2007). Other times they live much longer than their cooling time and do not have a soft X-ray counterpart. It is difficult to reproduce all these different observational properties of EUV loops with only one 
heating model. The last few years of investigation suggest that more than one class of loops exists, possibly having different heating mechanisms at work.

EUV images of ARs also reveal bright dense patches, called "moss", which have a peak of emission at about $\log T=5.8$. Moss was first identified by TRACE (Berger et al. 1999; Martens et al. 2000) and it is believed to be the footpoints of soft X-ray loops (Fig. 3, right panel). Moss has a pressure which is generally $2-3$ times the pressure in loops, and its physical parameters (temperature, density, and emission measure) have only small variations over hours. The filling factor is in the range 0.1-1 (e.g., Antiochos et al. 2003; Tripathi et al. 2010; Brooks et al. 2011).

The EUV brightness of an AR is not only due to loop emission, but also due to a diffuse emission which is only about 20 $30 \%$ less intense than the former (Del Zanna \& Mason 2003). The physical properties of this emitting plasma are still unknown. One possibility is that ARs also contain almost empty magnetic loops which are not detectable as isolated structures by our instruments. At the same time, to estimate such emission is extremely important as it should be subtracted from the loop emission to properly infer its physical parameters. Considering the relative intensity just mentioned between loops and their background, we expect that this diffuse emission plays a role in the energy budget of an AR. Much work should still be done on this topic.

The thermal structure of loops is a very important parameter for ARs because it allows, and it is used to test models for coronal heating. Different results have been found, particularly for EUV loops, leading to a debate in the solar community, which has intensified in the recent years.

The core soft X-ray loops are mostly isothermal in the cross-field direction. This property, together with their stable appearance and the stable emission of moss, are consistent with steady or almost steady heating mechanisms (e.g., Porter \& Klimchuk 1995; Schrijver et al. 2004). However, problems arise when this model is used to reproduce the amplitude of the EUV stable emissions of the moss, or the lower temperature emission of loops in the EUV, where the match does not work so well anymore (e.g., Warren \& Winebarger 2007; Winebarger et al. 2011 and reference therein).

A long-standing debate on the cross-field thermal structure of EUV loops is probably reaching its end. Spectroscopic and imaging measurements infer both isothermal and multi-thermal existing structures. Some of the past results were due to the limited diagnostic capabilities of the imaging instruments (e.g., Weber et al. 2005) or the limited spatial resolution of spectrometers $\left(1^{\prime \prime}-2^{\prime \prime}\right)$. Today several observational results converge on a model with a very fine structure of loops, at the limit of the available resolutions. Some of the loop models push forward by assuming the existence of bunch of unresolved strands which make up the observed loops. These strands are crossfield isothermal at a given time but, because of the limited spatial resolution of the instruments, the resulting cross-field temperature structure of the loop can be multi-thermal (e.g., Klimchuk 2006).

Looking at the temporal evolution of EUV emission, we realize that loops live longer than their characteristic cooling time scale. This property plus the multi-thermal cross-field structure, the filling factor, and the indication that they are overdense with respect to the same loop in static equilibrium, can be explained with a heating acting impulsively over small, probably unresolved scales (e.g., Cargill 1994; Klimchuk 2006;
Klimchuk et al. 2008 and references therein). Under this picture each strand is heated at a different time allowing, at a given moment, a different temperature in each strand. Due to the impulsive nature of the energy deposition, there will be few strands at very high temperature, while the majority will be cooling to temperature less than $1 \mathrm{MK}$.

Once this idea is also applied to the active region core loops, some consistent results are also found. Collections of impulsively heated loops (of hundred or thousands of seconds long) can reproduce the long stability of the emission and the isothermality of loops, or the cooling cycle observed by soft X-ray and EUV emissions in the same loops (e.g., Winebarger \& Warren 2005; Viall \& Klimchuk 2011), depending on the frequency repetition of the energy deposition in the strand compared to its cooling time. The high spatial resolution of the multi-band soft X-ray imager (XRT; Golub et al. 2007) on board Hinode has recently revealed its thermal diagnostic capabilities (Reale et al. 2007; Parenti et al. 2010). Consistent with small-scale heating, fine thermal structure over spatial scales was found comparable to that obtained with the EUV instruments.

In addition to these results, however, other observations of core loops are still in agreement with steady heating (Warren et al. 2011; Winebarger et al. 2011), or both heating mechanisms are possible (e.g., Tripathi et al. 2011). The question of heating in active region cores is still debated.

An observational aspect has been recently identified as an element that with no ambiguity can distinguish between steady and impulsive heating. This is the so-called "hot plasma" (>3 MK), which provides very faint emission identified in non-flaring active regions. This is measured as a diffuse emission of only a few percent of the main $3 \mathrm{MK}$ coronal emission. Figure 3 (right panel) shows a clear example. This plasma is predicted by the small-scale heating models as the result of the initial impulsive energy deposition on sparse strands hidden in the already cooling main bulk of the loop threads. This hot component is not consistent with steady heating.

The faint emission characteristic of this hot plasma makes its diagnostics difficult. For this reason few observations exist, and it has not been yet fully characterized (e.g., Reale et al. 2009; Goryaev et al. 2010 and references therein). At the same time, simulations show that this emission better conserves the properties of the heating energy, representing a good diagnostic tool (Parenti et al. 2006; Parenti \& Young 2008).

The difficulty of detecting it also may be due to its absence in some active regions, and it may be localized only in some part of the structure. The existing results suggest that its presence may depend on the age of the active region, but concrete evidence does not exist yet. Further investigations on this topic are needed and will certainly advance our knowledge on active regions behavior, their temporal evolution, their stability, and the loss of it. This includes establishing the repartition of the stored energy released during flares and eruptions, with important consequences on our understanding of Space Weather effects.

Mass flow exchange between active regions and the underlying chromosphere is a key element that should be fully pictured to properly understand the energy budget in corona. Spectroscopic EUV lines formed at chromospheric and transition region temperatures inside loops are systematically observed to be red-shifted indicating speeds of few tens of kilometers per second. The amplitude has a maximum of about $20 \mathrm{~km} \mathrm{~s}^{-1}$ and it is symmetric at the footpoints, although 
line-of-sight effects can affect the measurements. This flow generally decreases with increasing temperature (Teriaca et al. 1999; Dammasch et al. 2008).

At the same time, blue-shifted lines are observed at the outer boundary of the loops, where the emission is very weak (e.g., Del Zanna 2008; Hara et al. 2008). This flow increases with the temperature up to about $30 \mathrm{~km} \mathrm{~s}^{-1}$ for $\log T=6.3$, which is the present instruments limit for non-flaring emission. It is still not clear if this flow belongs to close field loops at low density, and for this reason not visible in the observations, or if it belongs to open field regions that are the source of slow wind. Upflows and downflows in non-flaring loops are signatures of chromospheric draining and evaporation and are typical of a dynamic heating process such as the impulsive heating on a small scale. These phenomena recall observational aspects commonly observed during flares, which made the idea that similar energy release processes may happen over a wide scale.

Siphon flows are also identified in loops. This is recognized as a mass flow which moves from one footpoint to the other as the result of pressure difference between the two footpoints. It has the characteristics of being supersonic (e.g., Teriaca et al. 2004; Bethge et al. 2012 and references therein) and it may be the signature of a localized heating event at one footpoint. In this context, we recall that Guglielmino \& Zuccarello (2011) found a countersiphon flow at the edge of a pore in the photosphere. A small-sized $(\sim 6000 \mathrm{~km})$ arch-shaped structure had a $4 \mathrm{~km} \mathrm{~s}^{-1}$ upward motion in the footpoint with stronger field strength, and downflows of the same order in the opposite footpoint.

More information on non-flaring active regions properties can be found in Reale (2010).

\section{Magnetic helicity transport and eruptive phenomena in the solar atmosphere}

In this Section we present recent studies that concern the role of magnetic helicity transport in eruptive phenomena that are crucial for Space Weather.

A useful quantity to investigate the degree of complexity reached by an AR in corona during its life, and in particular during the phases prior to eruptive phenomena, is the magnetic helicity $H=\int_{V} \mathbf{A} \cdot \mathbf{B} d V$, where $\mathbf{A}$ is the magnetic vector potential and $\mathbf{B}=\boldsymbol{\nabla} \times \mathbf{A}$ is the magnetic field (Berger \& Field 1984). This global parameter of the considered portion of the corona quantifies how much a set of magnetic flux tubes is sheared and/or wound around each other. In a plasma having high magnetic Reynolds number, the helicity is almost conserved on a time scale smaller than the global diffusion time scale, even taking into account the effects of magnetic reconnection. Therefore, in the solar corona the magnetic helicity can be injected only through the photosphere by new emerging flux or by horizontal motions of the field line footpoints, while its excess can be expelled only by CMEs (Demoulin \& Pariat 2009). However, it is not easy to estimate the magnitude of the magnetic helicity because it depends on the 3D configuration of the magnetic field in the corona which cannot be measured directly. Therefore, many researches have derived only the photospheric rate of the magnetic helicity injection into the corona.

Berger \& Field (1984) derived the Poynting theorem for the helicity in an open volume:

$$
\frac{\mathrm{d} H}{\mathrm{~d} t}=\oint 2\left(\mathbf{B}_{\mathbf{t}} \times \mathbf{A}_{\mathbf{p}}\right) v_{\mathrm{z}} \mathrm{d} S+\oint-2\left(\mathbf{v}_{\mathbf{t}} \times \mathbf{A}_{\mathbf{p}}\right) B_{\mathrm{z}} \mathrm{d} S,
$$

where $\mathbf{B}$ is the magnetic field, $\mathbf{v}$ is the velocity field (the subscripts $t$ and $z$ indicate components parallel and normal to the photosphere, respectively), and $\mathbf{A}_{\mathbf{p}}$ is the vector potential of the potential field, which is uniquely specified by the observed flux distribution on the surface with the equations:

$$
\nabla \times \mathbf{A}_{\mathbf{p}} \cdot \hat{\mathbf{z}}=\mathbf{B}_{\mathbf{z}}, \nabla \cdot \mathbf{A}_{\mathbf{p}}=0, \mathbf{A}_{\mathbf{p}} \cdot \hat{\mathbf{z}}=0 .
$$

According to Eq. 1, the helicity of magnetic fields in an open volume may change either by the passage of helical field lines through the surface (the first term) or by the shuffling horizontal motion of field lines on the surface (the second term, Chae 2001). A first method of determining the magnetic helicity flux using a time series of line-of-sight magnetograms has been proposed by Chae 2001. In this case the total flux was computed by the integral of the helicity flux density $G_{\mathrm{A}}=-2\left(\mathbf{A}_{\mathbf{p}} \times \mathbf{v}_{\mathbf{t}}\right) B_{\mathrm{z}}$ over the analyzed region, where the vector potential of the magnetic field $A_{p}$ was computed by means of the Fourier Transforms, the horizontal photospheric velocity was determined by the local correlation tracking (LCT) method (November \& Simon 1988), and the magnetic field component normal to the photosphere $B_{\mathrm{z}}$ was determined from the line-of-sight magnetograms.

More recently, Pariat et al. (2005) have developed a new method that reduces the presence of spurious signals in the magnetic helicity flux density. They defined a new proxy of the helicity flux density:

$$
G_{\theta}=-\frac{B_{\mathrm{z}}}{2 \pi} \int_{S^{\prime}} \frac{\mathrm{d} \theta(\mathbf{r})}{\mathrm{d} t} B_{\mathrm{z}}^{\prime} \mathrm{d} S^{\prime}
$$

where $\mathbf{r}$ is the vector between two photospheric points $x$ and $x^{\prime}$; consequently, $\mathrm{d} \theta(\mathbf{r}) / \mathrm{d} t$ is the relative rotation rate of these points, $B_{\mathrm{z}}=B_{\mathrm{z}}\left(x^{\prime}\right)$, and $S^{\prime}$ is the integration surface. In this case, the computation of the vector potential is avoided. A further step for the real estimation of the magnetic helicity flux is provided by the use of new methods for the determination of magnetic footpoint velocities from a sequence of line-of-sight magnetograms. For example, Schuck (2005) developed a new technique, named differential affine velocity estimator (DAVE), which applies an affine velocity profile to a windowed aperture that is consistent with the magnetic induction equation. The DAVE method was also implemented as a replacement of the LCT method in the magnetic helicity flux computations.

These methods have been applied to MDI line-of-sight magnetograms to study not only the role of $\mathrm{d} H / \mathrm{d} t$ in some case studies of eruptive events, but also some statistical evidence of the relationship between the rate of the magnetic helicity injection in the solar atmosphere and flares or CME occurrence (LaBonte et al. 2007; Park et al. 2008, 2010).

In order to provide a contribution to the debate about the role of the magnetic helicity in active filament formation and destabilization (see Sect. 5), Romano et al. (2009) applied different methods to determine the helicity sign and the chirality of the filament magnetic field and computed the magnetic helicity flux rate at the filament footpoints. They analyzed a filament which started to form on October 15, 1999, between AR NOAA 8731 and AR NOAA 8732, and erupted on October 20, 1999, between 5:50 UT and 6:50 UT (see Fig. 4). This event occurred at the same time of a North-West CME observed by $\mathrm{SOHO} / \mathrm{LASCO} \mathrm{C} 2$ and $\mathrm{C} 3$ since 6:26 UT to 11:18 UT. They showed a correspondence between several signatures of the sinistral chirality of the filament and of the 


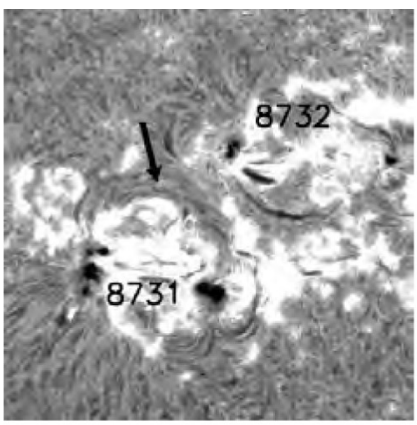

(a)

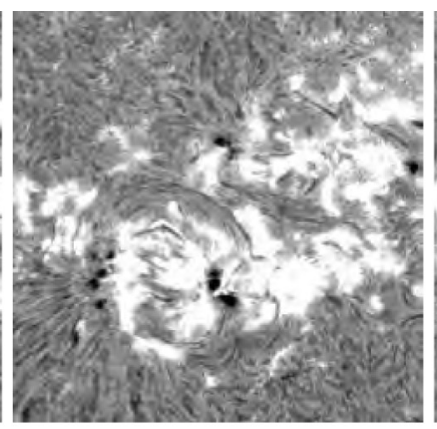

(b)

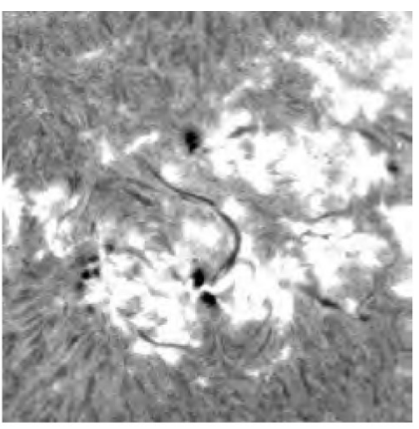

(c)

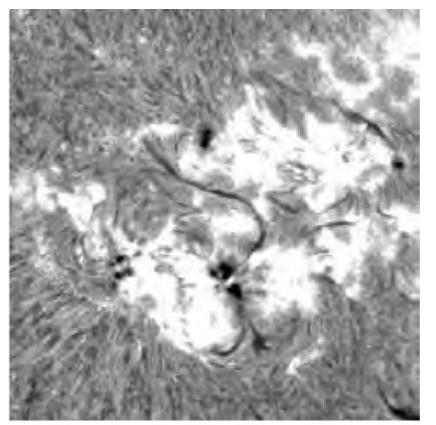

(d)

Fig. 4. Sequence of $\mathrm{H} \alpha$ images of two active regions (NOAA 8731 and NOAA 8732) acquired by Big Bear Solar Observatory on 15(a), 16(b), 18 (c), and 19(d) October 1999. The arrow in (a) indicates the location where a filament started to form. In (c) it is possible to see a barb of the filament. The field of view is $\sim 280 \times 280 \mathrm{Mm}^{2}$. North is on the top and West at the right.

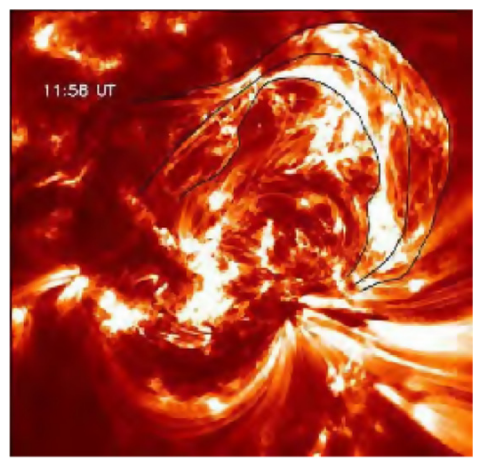

(a)

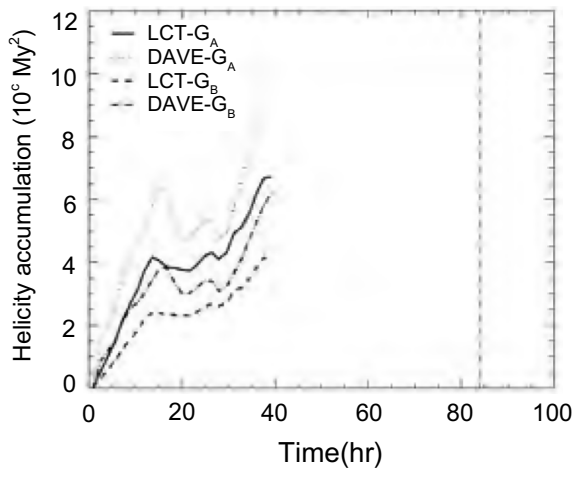

(b)

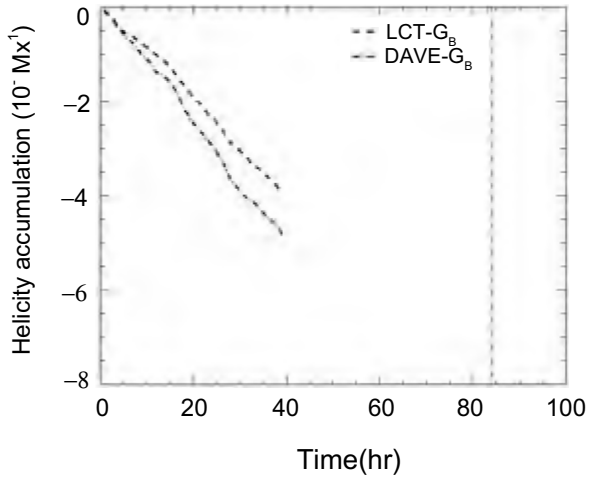

(c)

Fig. 5. (a) $171 \AA$ image taken by TRACE showing the EUV channel corresponding to the filament involved in the eruption; during the early phase of the eruption, a highly twisted arcade becomes visible; (b) and (c) magnetic helicity accumulation of the whole active region vs. time during two different data sets of MDI.

positive helicity of the filament magnetic field. They also found that the magnetic helicity transported along the filament footpoints showed an increase just before the change of the filament shape observed in $\mathrm{H} \alpha$ images. They argued that the photospheric regions where the filament was rooted might be the preferential ways where the magnetic helicity was injected along the filament itself and where the conditions to trigger the eruption were yielded.

Romano et al. (2011) studied a filament eruption on November 1, 2001, in NOAA 9682. Using BBSO $\mathrm{H} \alpha$ and TRACE $171 \AA$ images, as well as MDI line-of-sight magnetograms, they tried to highlight a specific aspect of the magnetic helicity accumulation, providing new observational evidence of the role played by the interaction of magnetic fields characterized by opposite helicity signs in triggering solar eruptions. Romano et al. (2011) used both the LCT and DAVE to determine the horizontal velocities and the two mentioned methods for estimating the magnetic helicity flux. The chirality signatures of the filament involved in the eruption were ambiguous, and the overlying arcade visible during the main phase of the event was characterized by a mixing of helicity signs (see Fig. 5). However, the measures of the magnetic helicity flux allowed to deduce that the magnetic helicity was positive in the whole active region where the event took place, while it was negative near the magnetic inversion line where the filament footpoints were located. These results suggested that the filament eruption might be caused by magnetic reconnection between two magnetic field systems characterized by opposite signs of the magnetic helicity.

Zuccarello et al. (2011) studied the same event with the aim to verify a possible relationship between the filament expansion and the helicity transport at its footpoints, i.e., if the variation in the magnetic helicity flux rate could be interpreted as a consequence of the magnetic torque imbalance caused by the tube expansion, as proposed by Chae et al. (2003). They used $171 \AA$ TRACE data to measure some geometrical parameters of the new magnetic system produced by the filament eruption and MDI magnetogram data to measure the accumulation of the magnetic helicity in the corona before and after the event. In contradiction to the expectations from the model described by Chae et al. (2003), the helicity injection after the eruption was positive. Therefore, they offered the alternative interpretation that the helicity injection resulted from torque of the opposite sign, generated as the filament lost its negative helicity through magnetic reconnection with its surroundings.

A wider sample of events was considered by Smyrli et al. (2010). They studied 10 ARs that gave rise to 12 halo CMEs observed by LASCO during the period 2000 February 2003 June. They found that the magnetic helicity flux does not have a unique trend in the events analyzed: in $40 \%$ of the cases it showed a large sudden and abrupt change that was correlated in time with a CME occurrence (see, e.g., 


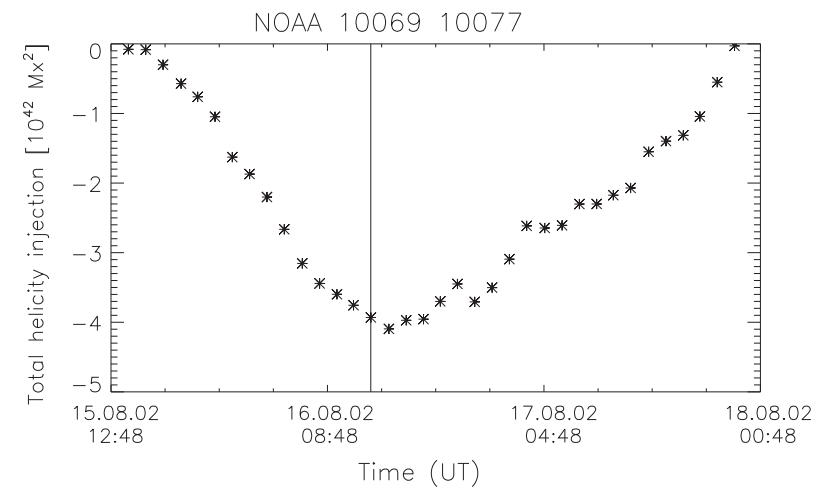

Fig. 6. Magnetic helicity accumulation in active regions NOAA 10069 and NOAA 10077 vs. time. The vertical line indicates the time of a CME occurrence (adapted from Smyrli et al. 2010).

Fig. 6), while in the other cases it showed a steady monotonic trend, with a slight change in the magnetic helicity at CME occurrence. Their results indicate that major changes in the magnetic helicity flux are observed in ARs characterized by emergence of new magnetic flux and/or generating halo CMEs associated with X-class flares or filament eruptions. In some of the analyzed cases the changes in the magnetic helicity flux followed the CME events and could be attributed to a process of restoring a torque balance between the sub-photospheric and the coronal domain of the flux tubes.

The amount of magnetic helicity injected into the corona through the photosphere in another sample of ARs during their passage across the solar disk was measured by Romano \& Zuccarello (2012). In this case the temporal variation of the maps of magnetic helicity flux was analyzed by measuring the fragmentation of the patches that were characterized by the same sign of magnetic helicity flux. The temporal correlation between the number of these patches and the flare and CME occurrence was studied. The fragmentation of the patches singled out in the maps of the magnetic helicity flux provided a useful indication of the evolution of the AR complexity. The more fragmented the maps of the magnetic helicity flux were, the higher was the flare and CME frequency. Moreover, most of the events occurred for low values (3-17) of the difference of the number of patches with opposite signs of the magnetic helicity flux (see Fig. 7). These results indicate that not only the accumulation of the magnetic helicity in the corona, but also its positive and negative fragmentation and distribution should be taken into account to provide a more confident indication of $\mathrm{AR}$ complexity and flare/CME productivity.

Taking into account all these results, Romano \& Zuccarello (2012) concluded that in the next future it would be important to make additional efforts to understand the contribution of magnetic helicity flux and of its distribution on the photosphere to the conditions for the occurrence of flares and CMEs. Progress in this field is extremely important in order to be able to predict the occurrence of phenomena having a strong impact on Space Weather conditions.

\section{Eruptive prominences: physical properties}

We report in this Section recent investigations on the physical conditions in the structures that erupt from the solar atmosphere.

Solar prominences or filaments (the two terms are used interchangeably for the same feature, depending on whether they are observed on the limb or on the disk, respectively) are formed along the photospheric polarity inversion line, also known as the neutral line, between regions of oppositely directed photospheric magnetic fields. They root down into the chromosphere or photosphere through the so-called barbs, which are features that extend from either side of the prominence spine (Martin 1998).

Prominence eruptions are one of the associated phenomena to CMEs and the debate on the role of prominences destabilization on CMEs initiation is still open. Prominences are coronal structures made up of dense plasma $\left(10^{9}<N_{e}<10^{11} \mathrm{~cm}^{-3}\right)$ at chromospheric temperature (e.g., Labrosse et al. 2010; Parenti et al. 2005a and references therein) and which is thermally and pressure isolated from the coronal environment. Magnetic field probably plays a major role in supporting and stabilizing these structures, which can persist for several solar rotations. These structures can become suddenly unstable and erupt, as previously discussed in Section 4.

The prominence-corona transition region (PCTR) also plays a major role to keep prominences core in thermal and pressure equilibrium. The emission of PCTR is stable for hours, suggesting that source of almost stable heating should be present to compensate these losses. This source is still unknown. Heating models can be tested once we know each term in the energy equation. The radiative losses are deduced from EUV emission by inverting the data to infer the differential emission measure (DEM). This gives the amount of plasma along the line of sight, at a given temperature. To properly constrain the inversion we need to sample the plasma in a wide temperature range. The most complete example is from Parenti \& Vial (2007) (see also Gunár et al. 2011) who used their prominence spectral atlas built on SOHO/SUMER data (Parenti et al. 2004, 2005b).

Figure 8 (left panel) shows the DEM for a quiescent prominence (Parenti \& Vial 2007), the middle and right panels the DEMs for a pre-erupting and erupting prominence observed by Kucera \& Landi (2008). We see how the thermal structure of the PCTR changes on the three different states of the structure. The quiescent prominence has a minimum of DEM at lower temperature than an activated and erupting prominence, and a shallower gradient at transition region temperatures. However, the corona background and foreground emission is not subtracted in the quiescent prominence, while this is the case for the other two DEMs. It is a rare case that data from the foreground and background coronal emission are available, so that the high transition region part of the PCTR is not well constrained. Until recently it was thought that very small amount of plasma from these structures was emitting at such temperatures. But the recent high signal-noise ratio data from SDO/AIA instrument is revealing the opposite. Faint emission is now visible from quiescent prominences in channels dominated by $\mathrm{Fe}$ VIII and Fe IX lines, suggesting that the emission comes from plasma which is at least at $4 \times 10^{5} \mathrm{~K}$ (Parenti et al. 2012). Very few measurements such as those shown in Figure 8 exist in the literature, and it is still not clear if these are characteristic patterns of the DEMs for a prominence in a different state.

Prominences fine structure is made of thin $(\sim 0 ! 3)$ threads observed in absorption on the solar disk in, e.g., $\mathrm{H} \alpha$ line (e.g., Lin et al. 2008). This measure, however, is imposed by the spatial resolutions achieved by the instrument. It is possible that thinner threads exist (e.g., Chae 2007). Unfortunately the UV-EUV instruments are still not able to reach such a spatial resolution on a regular basis (the limit is $1^{\prime \prime}$ ). Only sounding 

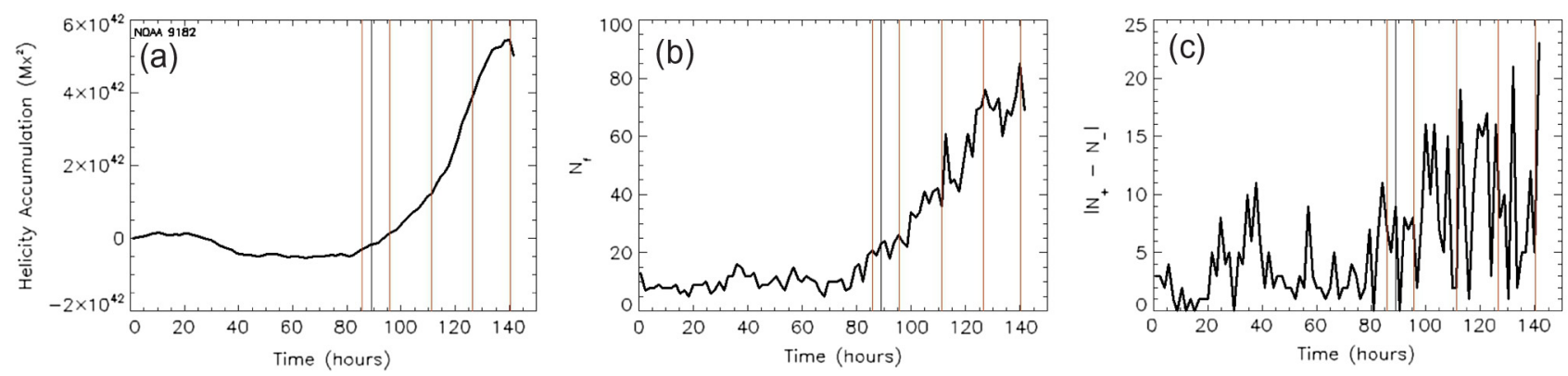

Fig. 7. (a) Accumulation of the magnetic helicity vs. time for NOAA 9182; (b) Number of patches identified in the map of the magnetic helicity flux vs. time; (c) Absolute value of the difference between the numbers of positive and negative patches vs. time. $t=0$ corresponds to 00:00 UT of the initial day of MDI data set used in the analysis. The vertical lines indicate the flare occurrence. The thickness of the vertical lines is equal to 1-3 for flares of GOES C, M, and X classes, respectively. In red are indicated the flares associated with CMEs.
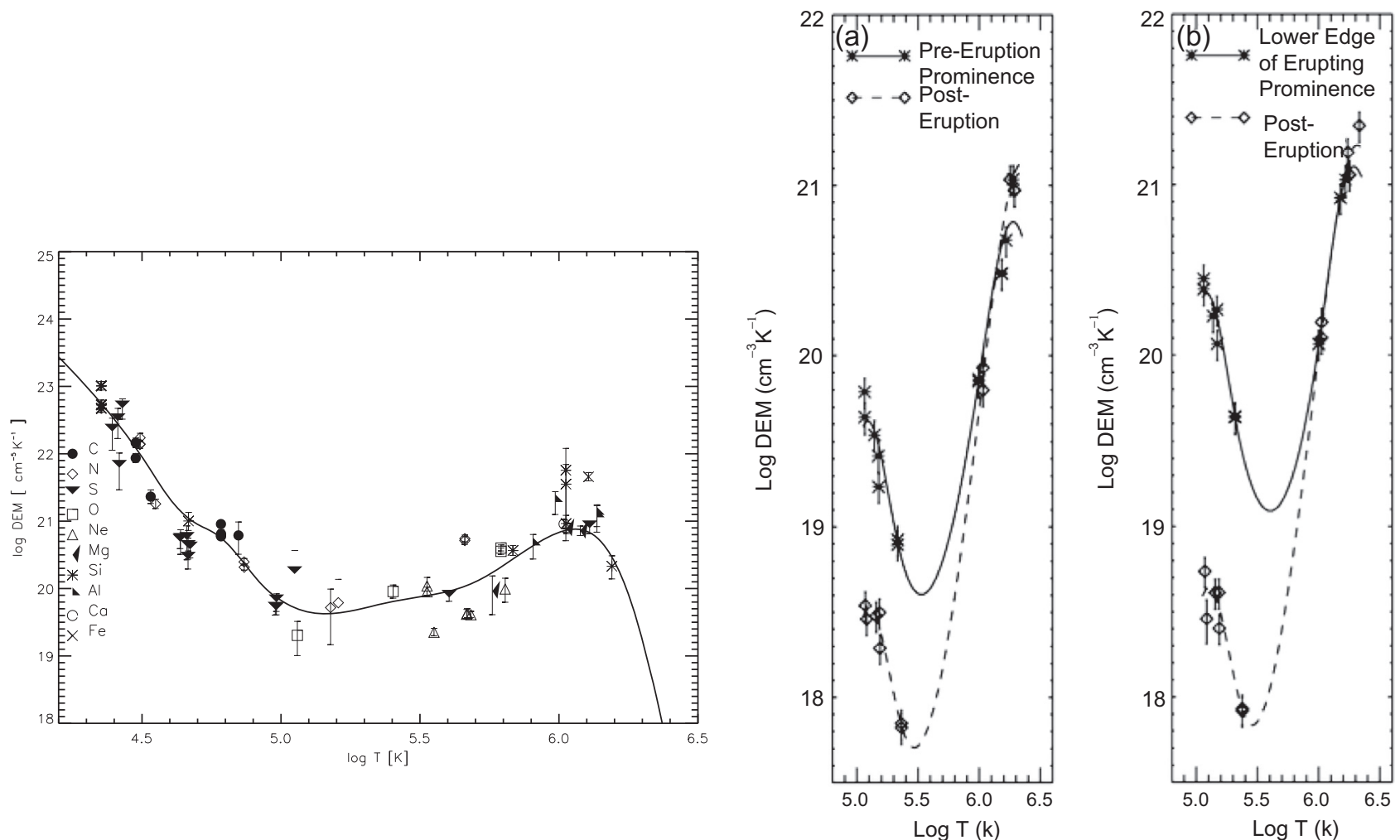

Fig. 8. DEMs for a quiescent (left) and pre (middle) and erupting (right) prominence. From Parenti \& Vial (2007) and Kucera \& Landi (2008).

rocket telescopes, such as VAULT, could reach 0 "15 imaging the Ly $\alpha$ line (Vourlidas et al. 2010; Vial et al. 2012). In these images, the fine structure appears slightly wider than in $\mathrm{H} \alpha$. There is no information at such resolution on hotter plasma. As a result we cannot state yet if each of these threads has its own PCTR or a unique PCTR envelopes the prominence cool core. There have been several attempts to answer this question, but the different methods used need to make strong assumptions (e.g., Cirigliano et al. 2004) and the question is still open.

Even in quiescent conditions the core of prominences show several dynamic manifestations. On the disk a counterstreaming motion of about $10 \mathrm{~km} \mathrm{~s}^{-1}$ is observed in the core threads and barbs (e.g., Engvold 1981; Schmieder et al. 1991). At the limb, dynamic threads, blobs, plumes which move upwards up to about $10-20 \mathrm{~km} \mathrm{~s}^{-1}$, or downstreams of the same amplitude are visible (Berger et al. 2008, 2011; Chae 2010). Threads oscillations are also common (Oliver 2009; Arregui et al. 2012).
These small-scale dynamics intensify once the prominence is activated.

In addition to these resolved motions, EUV spectral line width of quiescent prominences shows a broadening which exceeds the thermal width (Parenti \& Vial 2007). The associated speed increases from few kilometers per second at chromospheric temperatures to about $30 \mathrm{~km} \mathrm{~s}^{-1}$ in transition region. The origin of it may be wave motion, unresolved flows in multiple threads along the line of sight or microturbulence.

A filament may end its lifetime as a desparition brusque (Raadu et al. 1987; Schmieder et al. 2000), in which the filament diffuses slowly and disappears. Often prominences display a fast-rise phase during the eruption (Tandberg-Hanssen et al. 1980; Sterling et al. 2007), and sometimes they are also reported to follow a slow-rise phase before eruption (Schrijver et al. 2008), either with constant velocity (Sterling \& Moore 2005) or with constant acceleration (Joshi \& Srivastava 
2007). Sterling \& Moore (2004a, 2004b) have observed constant velocity for both the phases of filament eruption, and have attempted to fit models of reconnection to the observed events.

Most of the eruptive filaments are also known to be associated with CMEs (Gopalswamy et al. 2003; Webb \& Howard 2012 and references therein). Several explanations have been put forward for a better understanding of the two associated phenomena. For instance, Grechnev et al. (2006) considered the filament and CME eruption as a three-stage process with the help of a dual-filament CME initiation model. Vršnak et al. (1991, 1993) have observed a few prominences that showed helical twist during the eruptive stage. This was further studied by Srivastava et al. (1991), Srivastava \& Ambastha (1998), and Gilbert et al. (2007), who explained the helical structure by means of kink instability. Eruptive prominences also display asymmetric morphology, sometimes, i.e., one leg remains fixed in the lower corona, while the other leg detaches to erupt (Tripathi et al. 2006). CMEs, which are known to be closely associated with eruptive prominences, are also shown to exhibit twisted helical structures (Dere et al. 1999).

\subsection{Eruptive prominences and CMEs}

In this section we describe in detail the latest results about eruptive prominences and CMEs, that determine the solar forcing of the Space Weather.

It should be noted that almost all of the studies prior to the launch of the Solar TErrestrial RElations Observatory (STEREO) spacecraft (Kaiser et al. 2008) in 2006 on the twist of eruptive filaments and the two phases of rise were carried out either using ground-based data in $\mathrm{H} \alpha$ or data from a single spacecraft, mainly SOHO in EUV. The interpretation of the results obtained from these studies is constrained by inherent projection effects from a single viewpoint observation.

More recently, 3D studies have been carried out using observations from the identical Extreme UltraViolet Imager (EUVI; Howard et al. 2008) on board the twin STEREO spacecraft on various aspects of prominence dynamics (see Bemporad 2011 for a review). Gissot et al. (2008) and Liewer et al. (2009) carried out stereoscopic studies to obtain true coordinates and hence the true velocity of the prominence on May 19, 2007. Bemporad (2009) and Li et al. (2010) reconstructed several features of the observed prominence during its eruption to study the prominence shape as a whole. Joshi \& Srivastava (2011a) focused mainly on two aspects of the prominence eruption, i.e., the kinematics during the two rise phases and the helical twist of prominences during the fast-eruptive phase. The helical twist implies that the filament axis leaves its plane and forms a loop-like structure, such as in references Török \& Kliem (2005) and Chifor et al. (2006). There is another important manifestation of eruptive phase similar to the twist which is known as the "roll effect" (Martin 2003). During the roll effect, a prominence is seen to roll at the top, giving rise to twists in mutually opposite directions in the two legs of the prominence. Thompson (2011) reported on the rotation of a prominence about its direction of eruption by using a 3D reconstruction technique. Panasenco et al. (2011) also studied the rolling motion of three prominences and the associated CMEs from combined EUVI and COR1 observations.

STEREO/EUVI images have also been used by Gissot et al. (2008) who developed a fully automated optical-flow algorithm to estimate the height of an erupting filament from simultaneously obtained EUVI images from two viewpoints. Later,
Bemporad (2009) studied the 3D evolution of an eruptive filament using the EUVI images and inferred that the early filament expansion was anisotropic and occurred mainly on a plane parallel to the plane of the sky. Thompson (2011) traced out a filament in 3D space using EUVI images and found a rotation of $140^{\circ}$ from the original filament orientation. When the spacecraft separation became large, reconstruction of a polar crown filament in 3D was carried out by $\mathrm{Li}$ et al. (2011) using observations from three different viewpoints, namely the SDO, STEREO A and B spacecraft.

In a 3D study of two eruptive prominences, Joshi \& Srivastava (2011a) have reported their twisting behavior expressed quantitatively in terms of the changes in latitude and longitude of features selected along their legs (see Figs. 9 and 10). They found that the variation in longitude and latitude is due to an interplay of two motions: the overall non-radial motion of the prominence toward the equator and the helical twist in the prominence spine. This three-dimensional study of prominence kinematics also revealed two distinct phases of eruption: the slow-rise and the fast-eruptive phase, as previously reported by Sterling \& Moore (2005) and Joshi \& Srivastava (2007), both analyses based on projected planeof-sky observations.

Joshi \& Srivastava (2011a) have also shown that the values determined for different features along the prominences are different, but they are constant if just one feature is considered. The acceleration values in the fast-eruptive phase show strong grouping in each leg of the prominence in both the events analyzed. The net effect of the two motions, namely, non-radial propagation and helical twist in spine, produces a higher average acceleration $\left(11 \mathrm{~m} \mathrm{~s}^{-2}\right)$ in the western leg compared to the eastern leg $\left(5 \mathrm{~m} \mathrm{~s}^{-2}\right)$ in the prominence of April 13, 2010. While for the August 1, 2010 prominence, these two forces act to give rise to higher average acceleration in eastern leg $\left(20 \mathrm{~m} \mathrm{~s}^{-2}\right)$ compared with the western leg $\left(10 \mathrm{~m} \mathrm{~s}^{-2}\right)$. It is inferred that the magnitude of acceleration in the prominence legs is a response to the two dynamic motions experienced by them, which needs to be considered for a better understanding of prominence eruptions and their consequences on Space Weather conditions in future studies.

\section{Kinematics of eruptive prominences and associated CMEs}

In this Section we discuss the kinematics of eruptive prominences and associated CMEs, as this aspect is fundamental in order to distinguish between CMEs having important consequences on Space Weather from those that do not have such effects.

CMEs result from a loss of equilibrium in the magnetic configuration (Priest 1988; Klimchuk 2001). Several factors like flux emergence, flux cancellation, reconnection, shear, etc., can result in this loss of equilibrium (Forbes et al. 2006; Seaton et al. 2011). The source for the loss of equilibrium is generally considered to be magnetic energy associated with electric currents flowing in the corona. This energy is assumed to be sufficient for (1) opening the closed magnetic field lines at the CME site, (2) lifting the ejected material against the Sun's gravity, and (3) accelerating the material to typically several hundred kilometers per second (Forbes 2000; Alexander 2006). Initial statistical studies on kinematics of CMEs were carried out by Hundhausen (1993) and Hundhausen et al. (1994) using SMM and SOLWIND observations. More 

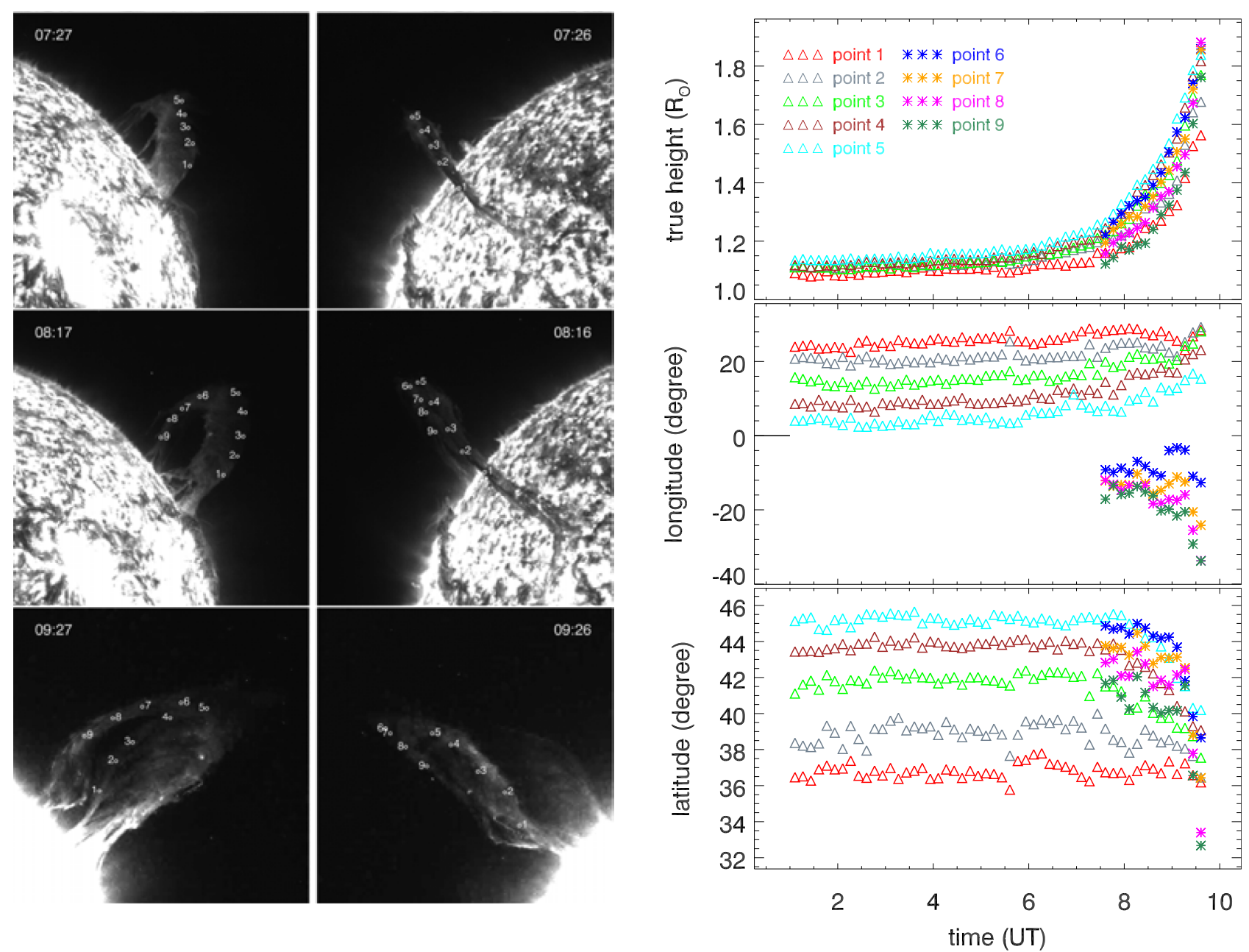

Fig. 9. Left: Erupting prominence on 2010 August 1 seen in $304 \AA$ images from EUVI B (left) and A (right) on board the twin STEREO spacecraft. Observation times in UT are shown for each image. The features used for reconstruction are marked and numbered along the prominence legs. Right: heliographic coordinates of different features of the prominence on 2010 August 1. Features 1-5 are from leg L1, while features 6-9 are from leg L2 of the prominence (adapted from Joshi \& Srivastava 2011a).

recently, Zhang \& Dere (2006) studied the kinematics of several CMEs and categorized the evolution of these phenomena as a three-phase process involving initiation, acceleration, and propagation (Fig. 11).

According to Zhang \& Dere (2006), the initiation phase is the phase of slow rise of CMEs; in the acceleration phase they undergo a very rapid increase in their velocity; while in the propagation phase the CME velocity remains more or less constant i.e., zero acceleration. Yashiro et al. (2004) also studied several CMEs from SOHO/LASCO observations and found that the CME velocity in the outer corona varies from less than $100 \mathrm{~km} \mathrm{~s}^{-1}$ to approximately $3000 \mathrm{~km} \mathrm{~s}^{-1}$. The propagation of CMEs can be understood if one considers the forces acting on them, i.e., the Lorentz force, gravitational force, and drag because of the ambient solar wind. Of the three forces, the drag force is the strongest beyond a few solar radii, and the other two can be neglected (Gopalswamy et al. 2001; Cargill 2004; Vršnak et al. 2010).

Gopalswamy et al. (2000) observed that although initial CME speeds range from $\sim 100$ to $\sim 1000 \mathrm{~km} \mathrm{~s}^{-1}$, the speeds of the corresponding interplanetary ejecta lie in the range of $320-650 \mathrm{~km} \mathrm{~s}^{-1}$, which is more or less the speed of the ambient solar wind. Cargill (2004) also reported that speeds of interplanetary CMEs (ICMEs) corresponding to CMEs with speeds ranging from 100 to $2000 \mathrm{~km} \mathrm{~s}^{-1}$, as measured from coronagraphs, lie within 100-200 $\mathrm{km} \mathrm{s}^{-1}$ of the ambient solar wind. However, the transit time, i.e., the time that a CME takes to reach the Earth, is known to vary from less than a day to over 4 days. Vršnak et al. (2010) reported that transit times of broad, low-mass CMEs depend mainly on the surrounding solar wind speed, while those of narrow, massive CMEs depend mainly on the initial speeds of the CMEs. Recently, Manoharan \& Mujiber Rahman (2011) have also found that most of the ICMEs tend to attain speeds close to that of the ambient solar wind. They estimated travel times of the CMEs based on their initial speed and drag due to solar wind.

CME kinematics have been classified on the basis of their source regions. It is well established that the eruption of solar prominences and CMEs is closely linked, as often the filament material erupts along with the CME. To study the dynamics of these closely associated phenomena together, kinematics of both the CME leading edge and the associated prominences need to be studied. In fact, Gosling et al. (1976), using data from space coronagraph on Skylab, for the first time report that CMEs associated with flares are faster than those associated with prominences. This was supported by observations of CMEs obtained using the K-coronameter at ground-based Mauna Loa Solar Observatory (MacQueen \& Fisher 1983). In addition, they also observed that flare-associated CMEs showed lower acceleration than the latter. 


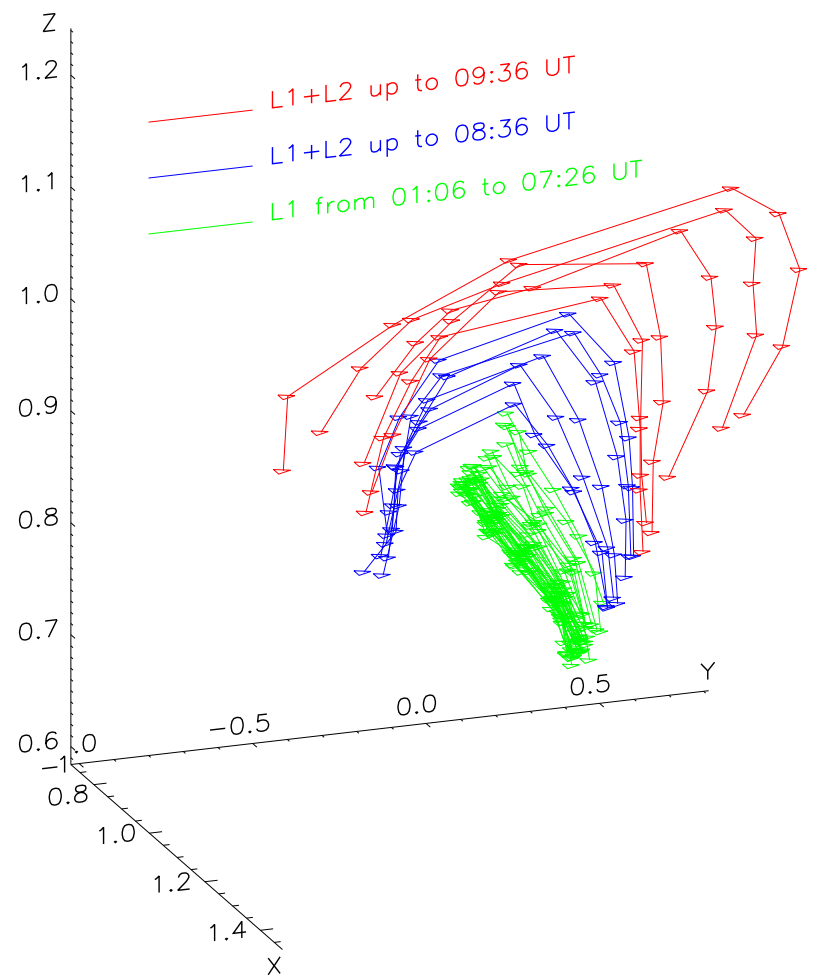

Fig. 10. Evolution of the erupting prominence shown in Figure 9 in 3D in heliographic coordinate system. The position of the prominence determined by joining all the reconstructed points at different instants of time, are marked on the plot. The coordinate system is centered on the Sun, with the $Z$-axis along the solar rotation axis, and the $X$-axis pointing toward the Earth (adapted from Joshi \& Srivastava 2011a).
Starting in 1996, the Large Angle and Spectrometric COronograph (LASCO) instrument (Brueckner et al. 1995), on board of the SOHO satellite, allowed for the first time to observe the corona with a large field of view (FOV), in the range of about 1.1-30 solar radii. A large number of CMEs have been tracked in all the three SOHO/LASCO coronagraphs.

Using these observations, Sheeley et al. (1999) reported similar results. Moon et al. (2002) in a statistical study involving over 3200 CMEs observed from SOHO/LASCO reported that flare-associated CMEs have a higher median speed than those associated with eruptive prominences. Their study also found that although the median acceleration of all the events is zero, it decreases for CMEs with high speeds $\left(>500 \mathrm{~km} \mathrm{~s}^{-1}\right)$. Srivastava et al. $(1999,2000)$ showed that gradual CMEs attain the speed of the ambient solar wind at about 20 solar radii from the Sun (see Fig. 12). Chen \& Krall (2003) also studied the acceleration of three CMEs using SOHO/LASCO observations and proposed that $\mathrm{CME}$ acceleration occurs in two phases, the "main" phase and the "residual" phase. While most of the acceleration occurs in the main phase, which is the interval over which the Lorentz force is the most dominant, there is a second phase of acceleration known as the residual acceleration in the outer corona. During the latter phase, the Lorentz force is comparable to the two other forces, viz, gravity and drag. In their model, Chen \& Krall (2003) proposed that the change in the duration of the flux injection (Krall et al. 2000) determines the strength of the residual acceleration phase.

The 3D observations of the solar corona taken by STEREO spacecraft have furthered our understanding of the CME kinematics. A recent analysis of six CMEs has been carried out by Joshi \& Srivastava (2011b) using coronagraphic observations by COR 1 and COR2, and the associated erupting prominences

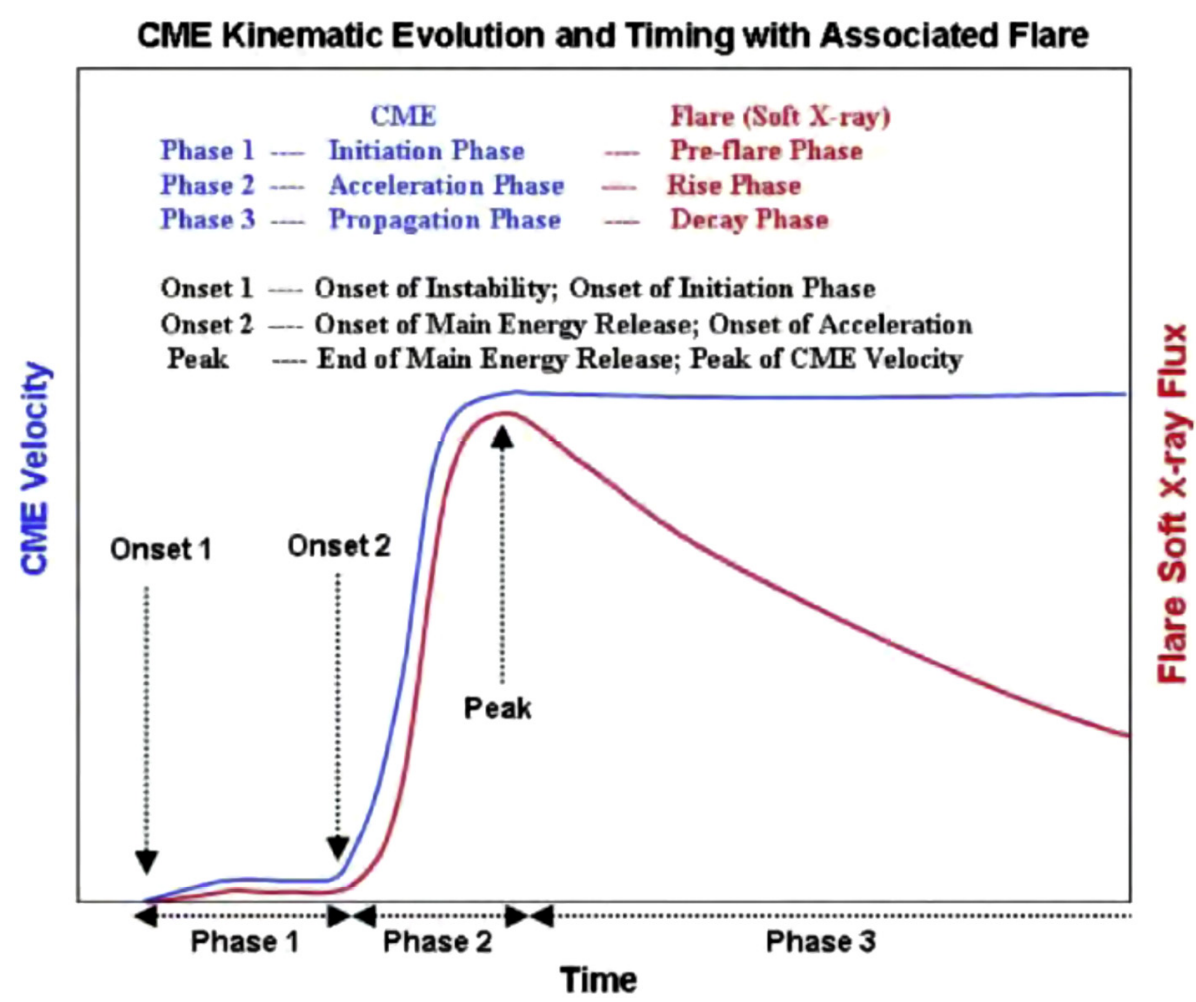

Fig. 11. Schematic plot of CME kinematic evolution and its relation with temporal evolution of GOES soft X-ray flare showing three distinct phases: initiation phase, acceleration phase, and propagation phase (Adapted from Zhang \& Dere 2006). 


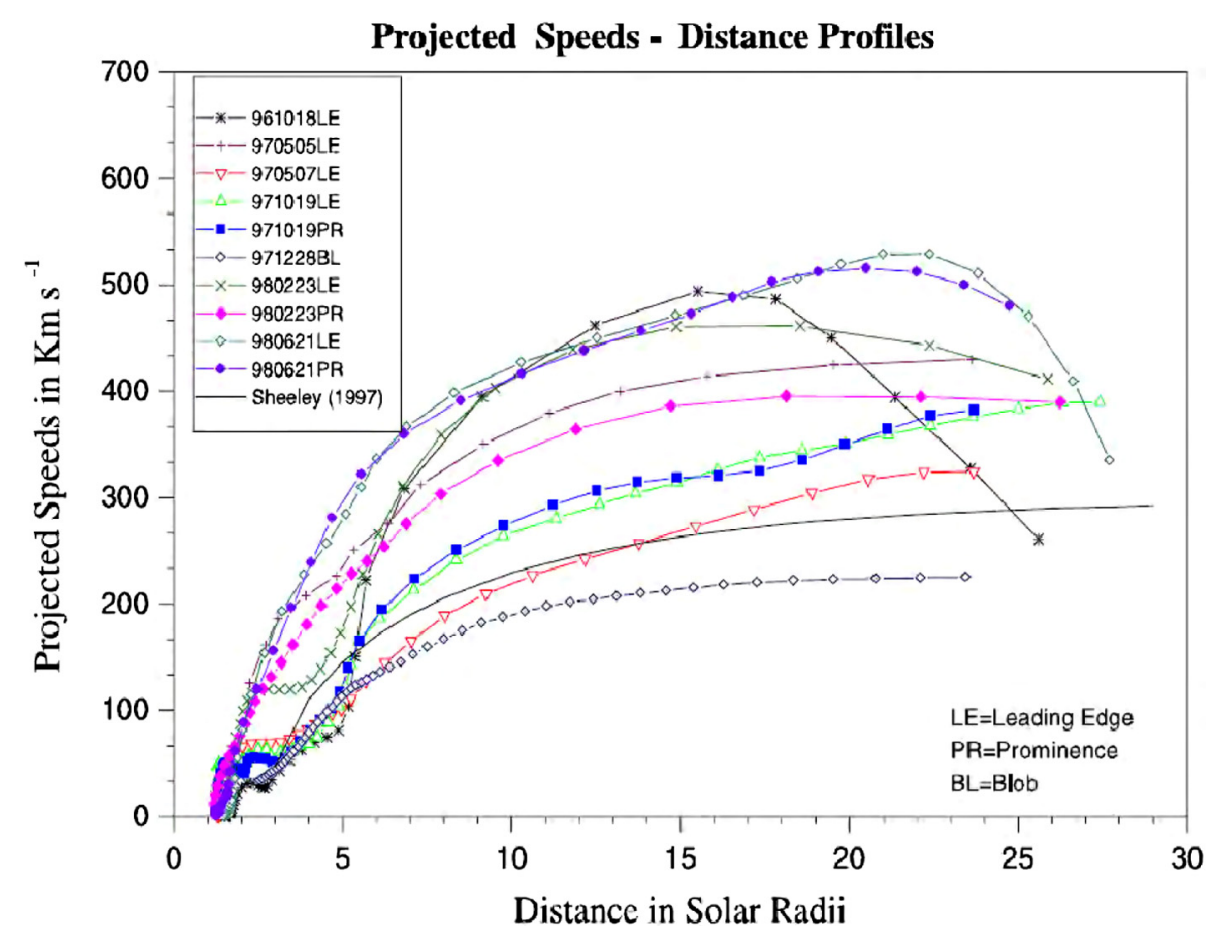

Fig. 12. Kinematics of different features of 7 CMEs observed by LASCO (Adapted from Srivastava et al. 1999).

in three of the cases observed by EUVI on board STEREO A and $\mathrm{B}$ spacecraft. While most of the earlier studies on CME acceleration were carried out using plane-of-sky measurements, they used 3D stereoscopic reconstruction technique, to obtain the true height, speed, and acceleration of selected CME features. It is to be noted that these true heights are true heliocentric distances and are significantly higher than the projected heights obtained from observations from a single spacecraft. Thus, although the FOV of the COR1 images is 1.4-4 solar radii, the true heights of selected features measured in these images will be higher and in most cases start close to 2 solar radii.

Using these true heights, the true speeds and acceleration of selected CME features were also estimated. In most of the events, the rise phase of acceleration of the leading edge of CMEs could not be captured and it was inferred that the peak of main acceleration lies much below the true height of 2 solar radii, as the acceleration values show a decreasing trend beyond this distance. Previous studies by Chen \& Krall (2003) show that the height of maximum acceleration of CMEs is less than 2-3 solar radii, while Vršnak (2001) considered this height to be 4 solar radii. More recently, Temmer et al. (2010) had estimated the height of maximum acceleration to be less than 2 solar radii. However, all of these studies are based on measurements of projected heights. Thus, 3D measurements indicate that most of the CME dynamics occurs closer to the Sun than previously suggested from single view point observations. Further, the estimates of maximum acceleration, average acceleration, acceleration magnitude, and acceleration duration, attained by the CMEs and erupting prominences, were compared, following the definition of Zhang \& Dere (2006). The 3D study by Joshi \& Srivastava (2011b) also reveals that the larger the acceleration, the higher up in the corona it occurs.

Previous studies based on projected height-time measurements reported that acceleration of a CME follows a bimodal distribution (Chen \& Krall 2003). Joshi \& Srivastava (2011b) have observed such a bimodal distribution in three CMEs (reconstructed in 3D) associated with flares. The residual acceleration ranged between 2 and $90 \mathrm{~m} \mathrm{~s}^{-2}$ for the three CMEs. The other CMEs, which are associated with erupting prominences, do not show such a bimodal acceleration implying that only the flare-associated CMEs undergo residual acceleration. This also supports the explanation given by Chen \& Krall (2003) that flux injection seems to be a good explanation for the eruption of the flare-associated CMEs.

STEREO mission observations have facilitated 3D studies of prominences specifically aiding in the determination of their true shapes and trajectories during eruption phase in three dimensions through stereoscopic observations made by EUVI and SECCHI coronagraphs. These observations take us a step further in understanding the prominence-CME relation and the prominence kinematics during eruption by explaining their twisted, helical motions. The determination of the true direction and heights of erupting prominence has been the main focus of recent studies using STEREO observations. For this purpose, different techniques have been developed and implemented on SECCHI observations. However, no success has been achieved with the 3D studies so far to resolve the question on the definitive role of magnetic reconnection (flare) on the eruption of a prominence (Bemporad 2011 and references therein).

\section{CMEs modeling}

CMEs, and especially fast CMEs, are one of the major drivers of the Space Weather. Certain magnetic topologies and preeruptive dynamics are more likely than others to produce fast CMEs. Therefore, understanding the physical mechanisms that determine the CME initiation is relevant in order to improve our knowledge in Space Weather. We review in the following the models used to describe this phenomenon.

In this regard, any model that aims to explain the origin of CMEs and of their trigger and driving mechanisms has to, at 
least qualitatively, explain the observed features, taking into account the pre-event constraints. The first constraint is the energy requirement. Physical considerations (Forbes 2010) show that the only energy reservoir that can actually account for the energy requirements of CMEs is the magnetic energy. However, how this energy is made available for the eruption is still an open question and the different ways in which this can happen distinguish the different $\mathrm{CME}$ initiation models. Observations show that during eruptions the photospheric magnetic field distribution does not change significantly, as a consequence the energy must be stored in the coronal magnetic field, and in particular into electrical currents. Therefore, all CME initiation models require a phase in which currents are present in the corona. There are models that address the way in which currents are built up into the corona (Mikic \& Linker 1994; Antiochos et al. 1999; Amari et al. 2000, 2003, 2010; Lynch et al. 2008) and models where this problem is not taken into account and that only focus on how the energy is released (Chen \& Shibata 2000; Lin \& Forbes 2000; Roussev et al. 2003; Török \& Kliem 2005; Lugaz et al. 2011).

CME initiation models are often classified in storage and release and directly driven models (Klimchuk 2001; Forbes et al. 2006; Forbes 2010; Chen 2011). In the storage and release models the magnetic energy is slowly stored into the coronal magnetic field up to a point when a threshold is reached or an equilibrium is perturbed and the field erupts. On the other hand, in the directly driven models the magnetic energy is pumped into the coronal system during the eruption itself. The flux injection model (Chen 1989) is maybe the most famous of the directly driven models. A flux rope, initially in equilibrium, can erupt as a consequence of a sudden injection of poloidal magnetic flux, i.e., current, from below the photosphere. The key feature in this model is that the magnetic flux has to be pumped inside the flux rope on time scales that are comparable with the time scale of the eruption, i.e., the coronal Alfvén time scale. However, the mechanism responsible for the eruption, i.e., the flux injection, is located below the photosphere and therefore can operate only on much slower time scales. This time scale paradigm is typical of all the directly driven models and this is the reason why, by now, the most accredited CME initiation models are based on the storage and release concept. During the storage phase the dynamics occurs on slower photospheric and sub-photospheric Alfvén time scales; however, the release phase occurs on the much faster coronal Alfvén time scales. This is in agreement with observations, where the pre-eruptive dynamics can last for some days before the occurrence of the CME.

The storage and release models can further be divided into two sub-classes: (1) models that do not require magnetic reconnection and (2) models that do require it (Howard 2011).

\subsection{Models not requiring magnetic reconnection}

This class includes models in which neither the trigger mechanisms nor the driver is somehow related to magnetic reconnection. However, magnetic reconnection can occur during the initiation process, but the initiation itself (trigger) and the acceleration process (driver) are not reconnection dependent. Furthermore, for this class of models the distinction between the trigger and the driving mechanism is not always clear. In fact, the eruption occurs as a consequence of a loss of equilibrium (mass loading/off-loading) or an MHD instability (kink/ torus instability).

\subsubsection{Mass loading/off-loading}

Due to its own weight, the plasma embedded in a magnetic structure can apply a downward directed force. This latter can balance the outward directed magnetic pressure that is generated when twist is injected into a flux rope. As a consequence more and more current, and therefore magnetic free energy can slowly be built up within the flux rope. At this point two scenarios can occur: (1) enough stress has been built up, the plasma weight cannot counterbalance the magnetic pressure anymore and thus the system erupts (Wolfson \& Dlamini 1997), or (2) due to the continuous plasma motions, as for example observed within the prominences, part of the mass if off-loaded, the plasma cannot balance any longer the magnetic pressure and an eruption occurs (Low 1996).

\subsubsection{Kink instability}

A current instability that can drive solar eruptions is the kink instability. Such instability occurs when a flux tube is twisted beyond a certain critical limit. When the critical limit is reached the tube becomes unstable and kinks (Roussev et al. 2003; Török \& Kliem 2003; Török et al. 2004; Rachmeler et al. 2009). Amari et al. (1996) considered the evolution of a bipolar magnetic field when both magnetic polarities are subject to vortex motions. After an evolution phase through a series of forcefree equilibria, the system undergoes a very fast opening. Török \& Kliem (2003) reconsidered a similar configuration and performed a series of numerical simulations in order to determine the existence of a possible threshold in the end-to-end twist of the flux tube after which no equilibrium can be found. The authors concluded that for a flux tube twist $\Phi$ between $2.5 \pi$ and $2.75 \pi$ the system undergoes a rapid expansion indicating that no stable equilibria exist anymore. Titov \& Démoulin (1999) presented a three-dimensional, line-tied, force-free flux rope model in which the degree of twist of the configuration can be controlled by the presence of a sub-photospheric imaginary current. Roussev et al. (2003) numerically investigated the equilibrium properties of such a configuration and found that even when the initial equilibrium is unstable, the flux rope cannot escape, unless the static arcade field associated with the line current is removed. Similar conclusions have been found by Török et al. (2004). These authors, considering a toroidal Titov \& Démoulin flux rope with an aspect ratio, i.e., the ratio between the torus major and minor radius, of five, found that the kink mode grows for a twist bigger than $3.5 \pi$. However, the presence of the overlying field inhibits the full eruption. Török \& Kliem (2005) reconsidered the problem and proved that kink unstable Titov \& Démoulin flux ropes can explain both confined and ejective eruptions depending on how fast the overlying field decreases with the height above the photosphere. Fan \& Gibson (2004) considered the emergence of a twisted flux tube in a pre-existing coronal field. They found that if the flux tube has a twist smaller than a critical value a stable force-free equilibrium containing a sigmoid can be obtained, but if enough twist is present the system undergoes a full eruption.

\subsubsection{Torus instability}

Ring currents are subjected to a repulsive (curvature) force, know as hoop force. Under the effect of this force a current ring tends to expand, but if a properly chosen extra magnetic field is included, an equilibrium can be obtained. However, this equi- 
librium is unstable and a small perturbation results in an increase of the ring radius. Kliem \& Török (2006) investigated the equilibrium properties of a current ring embedded in a dipole field. The authors found that if the dipole field scales with height as $r^{1.5}$, it cannot counterbalance the curvature force and the system undergoes an eruption. This current instability is known as torus instability and can be responsible for at least some prominence eruptions. Starting from a Titov \& Démoulin flux rope model, Török \& Kliem (2007) numerically investigate whether the torus instability can account for both fast and slow CMEs in both bipolar and multipolar active regions. They found that faster eruptions are obtained if the decrease of the overlying field is steeper, with the fastest ones occurring in multipolar active regions. Fan \& Gibson (2007), considering the emergence of a twisted flux tube, found that if the overlying arcade field declines slowly with height, the emerging flux rope remains confined until the threshold for the kink instability is reached and the system eventually erupts. However, if the overlying field declines more rapidly with height, the emerging flux rope is found to lose equilibrium and erupts via the torus instability. Recently, Aulanier et al. (2010) have performed a zero plasma- $\beta$ numerical MHD simulation of an asymmetric bipolar active region. By simultaneous applying slow magnetic field diffusion and sub-Alfvénic shearing motions in the photosphere, a stable slowly rising flux rope is created as a consequence of the reconnection in a bald-patch separatrix. When the flux rope reaches the altitude where the decay index for the potential field is about $3 / 2$, it undergoes a rapid upward acceleration. The authors concluded that photospheric flux-cancellation and tether-cutting coronal reconnection do not trigger CMEs, but contribute to both build up the flux rope and to make it rise up to the critical height above the photosphere at which the torus instability causes the eruption.

\subsection{Models requiring magnetic reconnection}

This class includes models where the magnetic reconnection is crucial in order to trigger and/or drive the eruption. The magnetic reconnection is a non-ideal process that allows to overcome the so-called Aly-Sturrock limit. Aly (1984) and Sturrock (1991) have argued that for a simply connected field, the fully opened magnetic field configuration always has a higher magnetic energy than the corresponding force-free field. Therefore, the Aly-Sturrock limit seems to imply that storage and release models are energetically impossible. However, this constraint can be overcome when non-ideal processes, such as magnetic reconnection, are involved. Magnetic reconnection is not the only way around this constraint. For example, an erupting configuration where only part of the magnetic field needs to be open (breakout model) also invalidates this limit. Finally, as discussed in the previous section, ideal-MHD processes can bring the flux rope all the way to infinity without opening the field.

\subsubsection{Flux cancellation model}

van Ballegooijen \& Martens (1989) proposed flux cancellation, driven by photospheric convergence motions toward the polarity inversion line, of a sheared arcade configuration, as a possible mechanism to transfer arcade field into helical field. The authors showed that, starting from an initial force-free magnetic field, a new magnetic field configuration in which the axis of the helical field moves to larger heights can be obtained by converging photospheric shearing motions. In an axisymmetric configuration, Forbes \& Priest (1995) considered the effect of convergence motions toward the polarity inversion line when a flux rope model (Forbes \& Isenberg 1991) is considered. When the photospheric magnetic sources approach each other, the magnetic energy stored in the flux rope increases until a critical point is reached and the system undergoes a loss of equilibrium.

Starting from the previous works of Forbes \& Isenberg (1991), Forbes \& Priest (1995), and Isenberg et al. (1993), Lin et al. (1998) analytically investigated the dynamics of line-tied flux ropes in an axisymmetric configuration around the Sun. They found that a loss of equilibrium occurs when the general dipole field is reduced below a certain value. As soon as the equilibrium is lost, a current sheet is formed. The flux rope eventually finds another equilibrium position that is several tens of solar radii away from the Sun. This loss of equilibrium does not fully open the magnetic field and the energy released is of the order of a few percent of the stored energy. However, if reconnection is allowed, the flux rope can undergo a full eruption and a significant amount of energy is released into the system (Lin \& Forbes 2000).

Starting from a two-dimensional flux-rope model, Chen \& Shibata (2000), in a Cartesian geometry, investigated the role that new magnetic flux emergence has in the destabilization of the flux rope. When an opposite directed magnetic field emerges below the flux rope, it cancels the pre-existing field and the flux rope starts to rise. However, when non-reconnecting flux emerges the flux rope finds a new equilibrium closer to the Sun. Dubey et al. (2006) extended this model including geometrical effects and the gravitational stratification of the surrounding medium. In a two-dimensional spherical geometry, Ding \& Hu (2008) considered the problem of the equilibrium of a flux rope, embedded in a background wind. Their simulations show that the emergence of new magnetic flux in the background field and far away from the flux rope itself eventually results in its destabilization. The catastrophic behavior of the system depends on the location and field orientation of the emerging arcade and on whether magnetic reconnection across the newly formed current sheet takes place.

The flux cancellation model has been also investigated in a Cartesian three-dimensional geometry of both bipolar (Amari et al. 2000, 2003, 2010, 2011) and multipolar Amari et al. (2007) active regions. Starting from a potential magnetic field generated by a dipole placed below the photosphere, Amari et al. (2003) applied photospheric twisting motions around the positive and negative polarities in order to build up current into the system. During this slow phase the system evolves through a sequence of force-free equilibria and is brought to a point where high sheared field lines are present near the polarity inversion line. Afterwards, converging motions toward the polarity inversion lines are also applied and the magnetic reconnection between the highly sheared field lines results in the formation of a twisted flux rope. They found that the rope is never in equilibrium and experiences a full eruption. In a recent paper, Amari et al. (2011) have reconsidered the same initial magnetic field configuration, but applied radially diverging flows centered around the two magnetic polarities instead of applying convergence motions overall the magnetic sources. In the region between the two magnetic polarities centers these flows converge toward the polarity inversion line. As a result an equilibrium including a flux rope is found. However, after some time, the system suffers a full disruption. This simulation shows that in order to drive an eruption, it is not necessary to advect the full AR toward the polarity inversion line. 


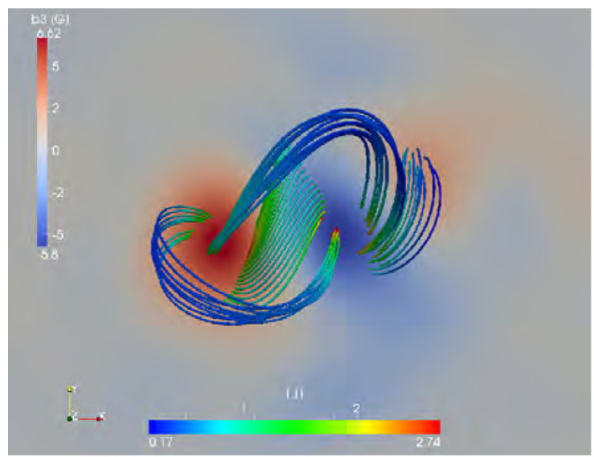

(a)

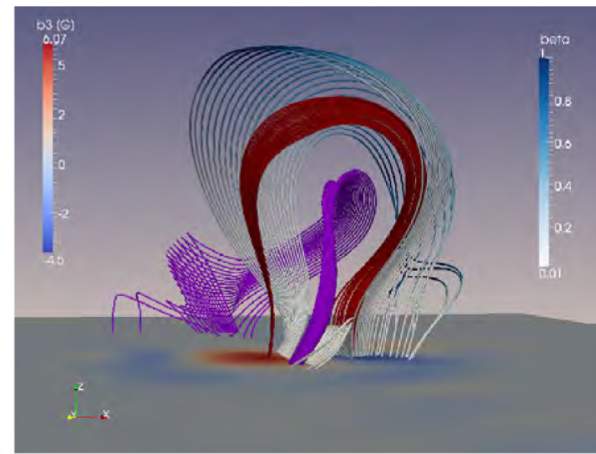

(b)

Fig. 13. Top and side views of the system when (a) the NLFF equilibrium is reached and (b) when the flux rope is formed. Selected field lines are color coded with the current density expressed in non-dimensional units (a) and plasma- $\beta$ (b). After Zuccarello et al. (2012b).

(a)

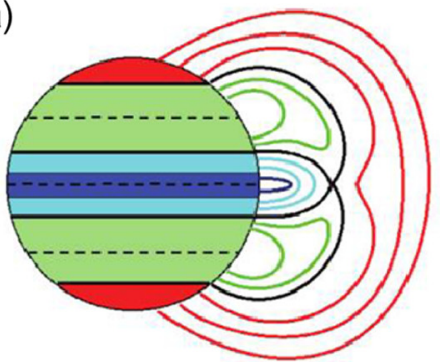

(b)

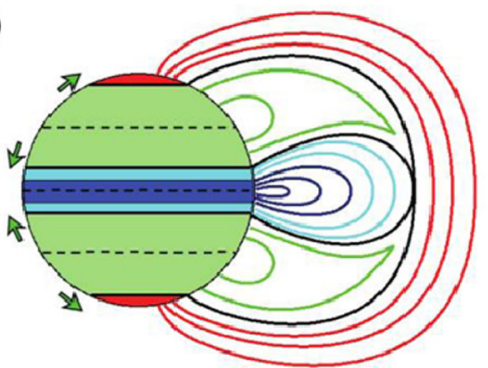

(c)

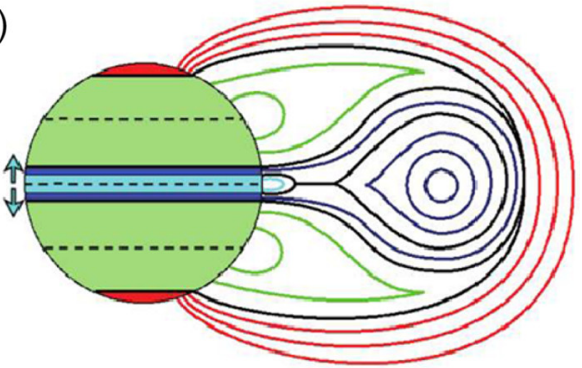

Fig. 14. Cartoon of the breakout model after Lynch et al. (2008). See text for a description of the model.

Starting from a current-free magnetic field extrapolation of AR NOAA 9415 embedded in a non-zero plasma- $\beta$ atmosphere, Zuccarello et al. (2012b) investigate the response of the solar corona when a new class of asymmetric convergence motions, resembling the ones observed for AR 9415, are applied at the inner boundary. The authors apply photospheric vortex motions along the isocontours of the vertical component of the magnetic field, in order to obtain a non-linear-force-free equilibrium having the same chirality as the one of the observed AR. Figure 13a shows the initial NLFF equilibrium, where high sheared field lines are observed close to the polarity inversion line together with $J$-shaped field lines bundles. As a consequence of the applied convergence motions, tether-cutting reconnection sets in between the highly sheared field lines, eventually resulting in the formation of a current-carrying flux rope (Fig. 13b). The tether-cutting reconnection transfers overlying field into axial field of the flux rope, increasing its magnetic pressure and driving the eruption. At the moment of the eruption about the $4 \%$ of the photospheric flux was canceled, supporting the conclusions of Amari et al. $(2000,2011)$ and the close relation between different classes of photospheric convergence motions and flux cancellation (Amari et al. 2010).

\subsubsection{The breakout model}

Based on the observational consideration that often CMEs originate from multipolar active regions, Antiochos et al. (1999) proposed the magnetic breakout model as a possible scenario for the CME initiation.

The key feature of the model is the requirement of a quadrupolar magnetic field distribution at the lower boundary. Figure 14a shows the 2D version of such a configuration. Four flux systems are present: (1) the overlying magnetic field (red field lines), (2) the central arcade field (light blue lines), and (3) the two side arcade magnetic fluxes (green lines). The general dipole field (red flux) is antiparallel with respect to the central arcade flux (light blue) so that a magnetic null $X$-point above the central arcade is formed. Finally, the figure also shows the separatrices between the different flux systems (dark blue lines) and the three polarity inversion lines (dotted lines). Magnetic energy is introduced into the system by applying photospheric shearing motions along the polarity inversion line of the central arcade, i.e., along the dark blue area on the solar surface. As a consequence of these motions, the magnetic pressure inside the central arcade increases and the arcade starts to expand (Fig. 14b), eventually compressing the $X$-point and forming a current sheet. At a certain moment, magnetic reconnection sets in at the $X$-point transferring flux from the overlying field toward the side arcades, reducing the magnetic tension of the overlying field, and facilitating the eruption. This magnetic reconnection is known in the literature as breakout reconnection. While the shear continues, the flanks of the central arcade pinch together and a second current sheet is formed. The magnetic reconnection at the flanks of the central arcade, known as flare reconnection, results in the formation of a flux rope. In the breakout model is the flare reconnection that actually accelerates the newly formed flux rope, possibly resulting in fast CMEs (MacNeice et al. 2005).

Starting from a breakout favorable magnetic field configuration embedded in the solar wind Zuccarello et al. (2008) investigated whether the emergence of new magnetic flux from below the photosphere could result in the formation of a CME. The emergence of new magnetic flux of the same polarity as the central arcade flux results in the expansion of this latter. As a 


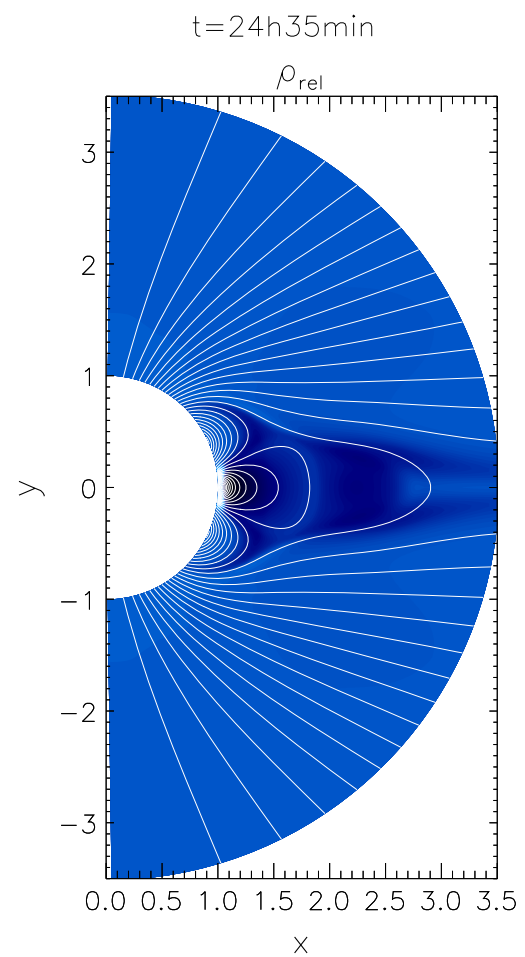

(a)

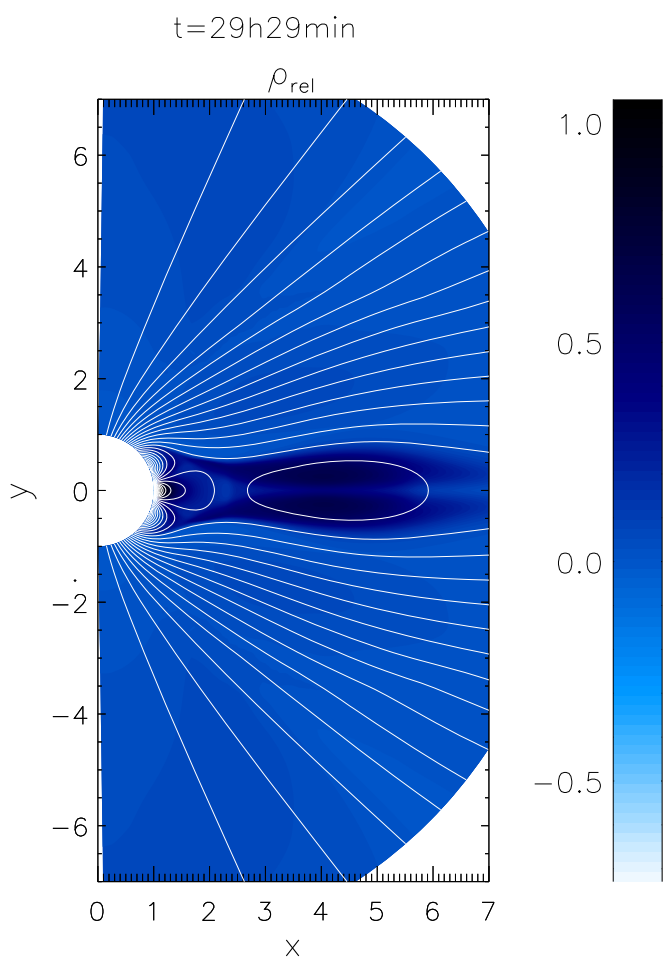

(b)

Fig. 15. Snapshots of the relative density and of the magnetic field lines for the simulation of Zuccarello et al. (2008).

consequence the breakout reconnection sets in at the top of the expanding central arcade resulting in the detachment of the helmet streamer that is carried away by the solar wind resulting in a slow blow-out CME, as shown in Figure 15. In a similar configuration, Zuccarello et al. (2009) investigated the mutual relation between the emergence of new magnetic flux and shearing motions as CME initiation mechanisms. They found that independently of the applied mechanism all eruptions turned out to be the detached helmet streamer and were all slow CMEs. They concluded that at least in an axisymmetric configuration the inclusion of the solar wind is the reason why no breakout CMEs have been obtained. Figure 16 shows two snapshots of the simulation of Zuccarello et al. (2009), where two flux ropes are visible: (1) the leading flux rope, i.e., the detached helmet streamer, and (2) the breakout flux rope formed from the central arcade, as a consequence of the applied shearing motions. The detached helmet streamer has a magnetic field vector rotating in a clockwise direction, while the magnetic field of the second flux rope has a counterclockwise orientation. At some point, the second flux rope will start reconnecting with the sides of the elongated helmet streamer, eventually transferring flux partially to the top of the helmet streamer and partly to the overlying field. Finally, the initiation of CMEs in an asymmetric breakout configuration was investigated by Bemporad et al. (2012). The obtained eruption is a breakout CME; however, due to the interaction with the solar streamers, this CME is again a slow one. See also Zuccarello et al. (2012a) for another example.

Among the models discussed, the breakout model and the Amari et al. (2000, 2003) model are the only ones that also address the problem of the flux-rope formation. In fact, all the previous models assume the presence of the flux rope and only investigate its stability properties. On the other hand, in these two models the flux rope is formed on the fly as a consequence of the shearing/twisting motions. This is both a positive and a negative aspect of the model. It is positive because gives insights into the flux rope formation, but unfortunately this process requires coherent, large-scale photospheric motions that are not observed in the solar photosphere. The inconsistency between the required photospheric motions and the observed ones is a common problem for most of the models and simulations presented in the literature, at least where a flux rope needs to be formed. Nevertheless, these models still give valuable explanation for the initiation and acceleration process. For example, for the breakout model, if the central flux system presents already a significant shear, while for instance the flux emerges from below the photosphere, the breakout reconnection will definitely facilitate the eruptions, eventually explaining faster CMEs (MacNeice et al. 2005; Lynch et al. 2008; van der Holst et al. 2009).

\section{Coronal Mass Ejections and 3D reconstruction techniques: three case studies}

As mentioned earlier, one of the aims of the COST Action is to progress in the scientific understanding of Space Weather. A particular aspect is the investigation of the evolution and propagation of solar structures, such as CMEs, recognized to be the main drivers of Space Weather, by performing a 3D reconstruction of CMEs from spacecraft observations.

The online CME catalog (http://cdaw.gsfc.nasa.gov/ CME_list/) contains data about the CMEs observed by the LASCO coronagraphs from the Earth direction. Nowadays, these data can be correlated to the ones taken by the two twin STEREO satellites (Kaiser et al. 2008), that allow a stereoscopic view of CMEs. 


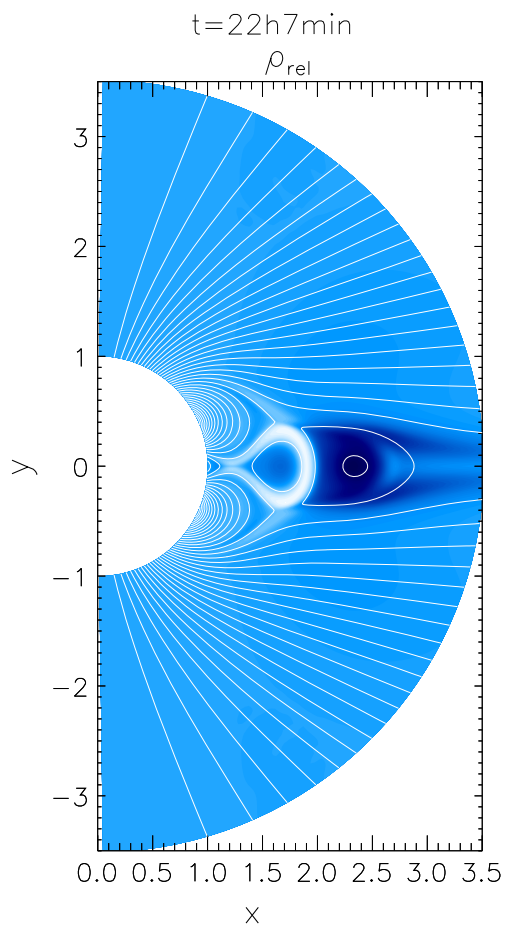

(a)

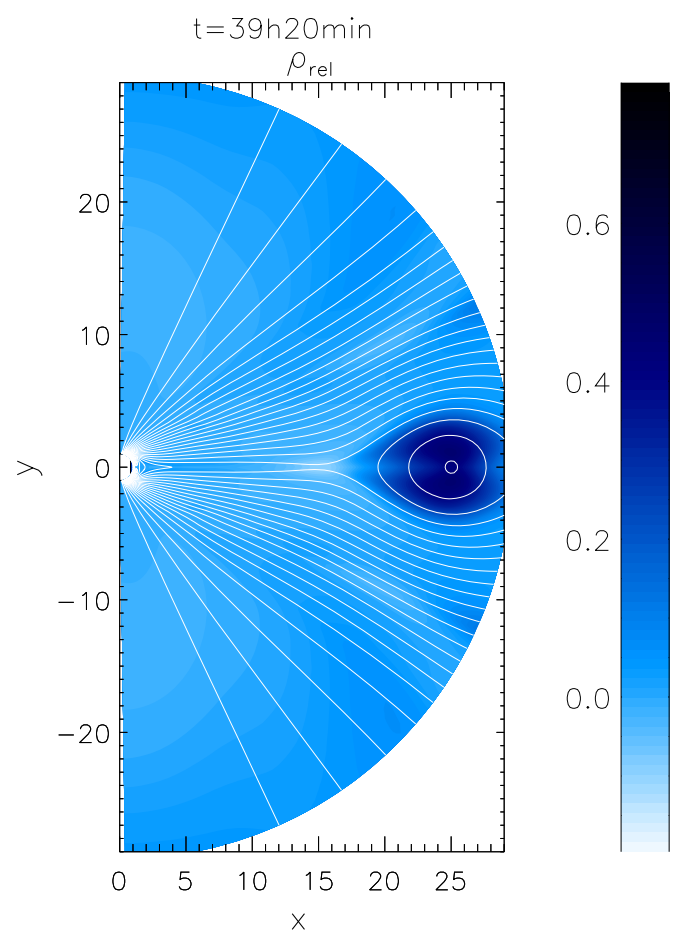

(b)

Fig. 16. Snapshots of the relative density and of the magnetic field lines for the simulation of Zuccarello et al. (2009).

\subsection{Description of 3D reconstruction techniques}

There are several ways to derive the position of a feature in 3D from two viewpoints (either from both STEREO spacecraft or from one STEREO spacecraft combined with SOHO), here we describe some of the most popular methods used to map features in the SECCHI-COR coronagraphs (Howard et al. 2008) and SECCHI-HI imagers (Eyles et al. 2009) datasets.

Three methods used to reconstruct features through COR images include: the triangulation method, the gradual cylindrical shell model, and the polarized ratio method. The triangulation method involves: finding a corresponding feature in two images (identified by either visual inspection or using a LCT method) and calculating where the lines of sight of the projected feature in each image intersect in 3D space. The intersection of the two lines defines the location of the feature (e.g., Inhester 2006). The gradual cylindrical shell model is a forward modeling (FM) technique (see Thernisien et al. 2006, 2009). This model is used to reproduce large-scale structures such as CMEs. For a flux rope-like CME a tubular section is often used to represent the main body and two cones for the legs. Only the surface of the CME is modeled, there is no rendering of its internal structure. Finally, the polarized ratio (PR) method, which is based on the Thomson scattering geometry (see Billings 1966), can be used to obtain a 3D reconstruction of a CME from coronagraph images obtained with different polarizer orientations (Moran \& Davila 2004; Dere et al. 2005; de Koning \& Pizzo 2011). The PR method can be applied separately to COR-A and COR-B images. By applying the method to both sets of images it is possible to get two different results that can be compared with each other, thus giving an estimate of the method uncertainty. These three methods are employed below to derive the 3D properties of CMEs in coronagraphs FOV. A more detailed description of the methods can be found in references Mierla et al. (2010) and Thernisien et al. (2011).

Methods for deriving the positions of ICMEs observed in HI observations include: the Fixed- $\Phi$, Point-P (Kahler \& Webb 2007), and Harmonic Mean methods (Lugaz et al. 2009). The first two methods involve identifying a single feature from both HI points of view and triangulating the position. However, in the HI FOVs, distances from the Sun are measured in elongation, $\epsilon$ (degrees), the Fixed- $\Phi$ and Point-P methods convert the position of observed features from elongation to radial distances, $r$ (AU). The Point-P method describes the region of maximum Thomson scattering by the ICME electrons as seen by the observer (Vourlidas \& Howard 2006), and is defined as: $r(P)=\sin (\epsilon)$ (see Kahler \& Webb 2007). The Fixed- $\Phi$ method uses the angle between the Sun and the propagating structure to track a feature. For a radial motion from a source at colatitude $\Phi$, the Fixed- $\Phi$ method gives the distance from Sun center (AU) as (Kahler \& Webb 2007):

$$
r(\Phi, \epsilon)=\tan (\epsilon)[\sin (\Phi)+\tan (\epsilon) \cos (\Phi)]-1 .
$$

The main difference between these two methods is: the CME is assumed to be expanding spherically from the Sun in the Point$\mathrm{P}$ method, whereas, the CME is considered to be a narrow structure propagating along a fixed direction (given by $\Phi$ ) in the Fixed- $\Phi$ method. The Harmonic Mean method is a technique developed by Lugaz et al. (2009) designed to complement the 3D fitting of CMEs by cone models or flux rope shapes. The method derives an analytical relationship between elongation angles and radial distances for CMEs, where the relationship is the harmonic mean of the Point-P and Fixed- $\Phi$ approximations. The two former methods are applied in this study. See Möstl et al. (2010) for an application of the Harmonic Mean method to the HI data. 


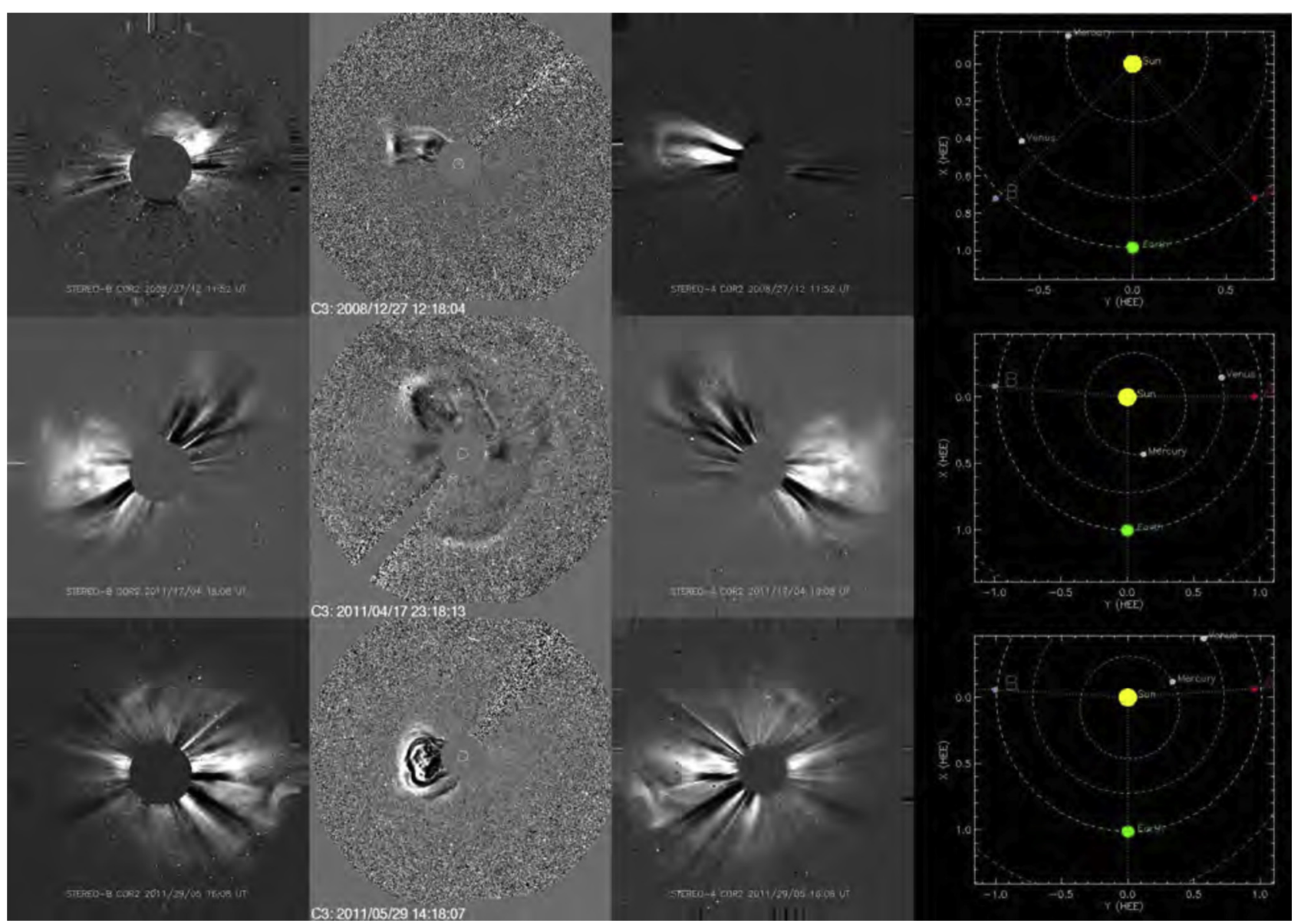

Fig. 17. Total brightness difference images with the three perspectives of the analyzed events. Top: December 27, 2008, center April 17, 2011, bottom: May 29, 2011. From left to right: COR2-B, LASCO C3, COR2-A, and the location of the three spacecraft.

\subsection{The case studies}

The polarization technique, introduced by Moran \& Davila (2004), allows the creation of "topographical maps" of the CME structure, which evidence the average height of plasma above/below the plane of the sky (POS). Through this capability it is possible not only to analyze the morphology of the CMEs, but also to calculate its central direction of propagation and speed in 3D space. The determined properties can be correlated with given characteristics of their respective source regions. This is important not only to improve the physical understanding of CMEs, but also to constrain models of CME morphology and propagation and to improve the current status of Space Weather forecasts. One of the advantages of this method of analysis is its validity for data acquired from the perspective of a single spacecraft. However, we profit from the two vantage points of the STEREO Mission to validate the methods and results.

Three events have been three-dimensionally characterized by analyzing polarized images acquired by the white-light coronagraphs COR1 and COR2 of SECCHI, aboard the STEREO Mission. The events were selected according to different location scenarios of the twin STEREO spacecraft, by investigating data available at the STEREO Science Center Website (http:// stereo-ssc.nascom.nasa.gov/), the STEREO COR1 CME Catalog (generated by O.C. St. Cyr and maintained by H. Xie from September 2007 onwards; see http://cor1.gsfc.nasa.gov/catalog/), and the CACTUS COR2 Catalog of Detections (Robbrecht \&
Berghmans 2004; Robbrecht et al. 2009; maintained by the Naval Research Laboratory (NRL; see http://secchi.nrl.navy.mil/cactus/). The cases of interest with particular relative locations of the A and B twin STEREO spacecraft, and SOHO providing a third view from Earth's perspective, are:

1. the A and B spacecraft approximately in quadrature, with both observing the same CME, one as a limb event and the other one as a halo event (December 27, 2008; headed to STEREO-B),

2. the A and B spacecraft $\sim 180^{\circ}$ apart, with both observing the same CME as a limb event, and with the CME observed as a halo event from the Earth's perspective (April 17, 2011; propagating away from SOHO), and

3 . the A and B spacecraft $\sim 180^{\circ}$ apart, with both observing the $\mathrm{CME}$ as a halo event, and as a limb event from SOHO's perspective (May 29, 2011; headed to STEREO-B).

Figure 17 shows, for each of the selected events (December 27, 2008: top; April 17, 2011: center; May 29, 2011: bottom), total brightness difference images of COR2-B (left column), SOHO/LASCO C3 (center-left column), COR2-A (center-right column), and the location of the A and B spacecraft for the corresponding dates of the events (right column).

The data sets were downloaded from the STEREO Science Center Website and include images from the SECCHI COR1 and COR2 instruments, taken at the three polarizer positions 


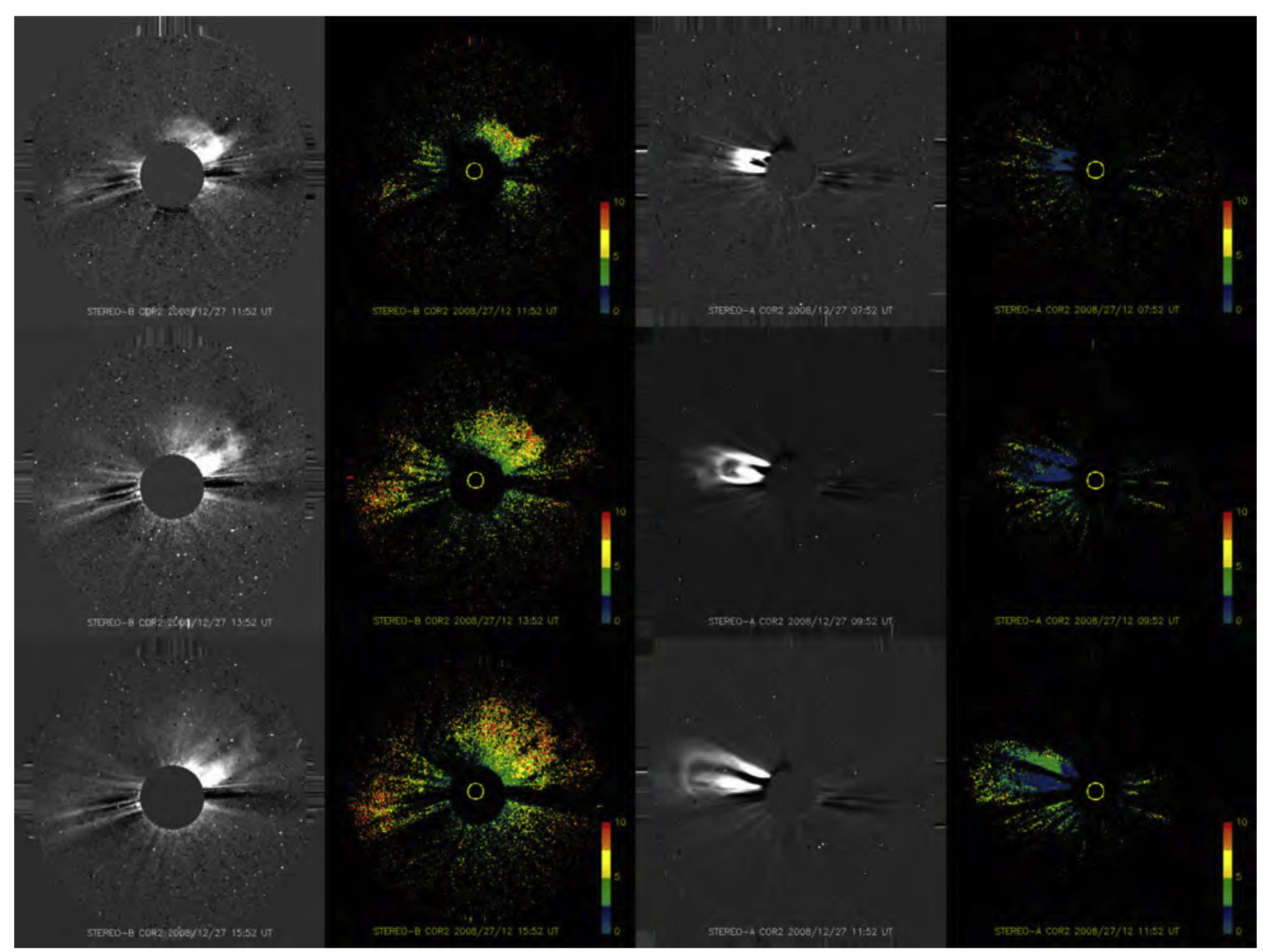

Fig. 18. Time sequence of the CME event on December 27, 2008. Columns 1 and 2 correspond to COR2-B, while columns 3 and 4 correspond to COR2-A. Images on columns 1 and 3 are base-differences, while columns 2 and 4 show heights with respect to the plane of the sky, after application of the polarization technique. The color bar indicates heights $|z|$ in units of solar radii.

$\left(0^{\circ}, 120^{\circ}, 240^{\circ}\right)$ and covering the full passage of the CMEs through the FOV of the coronagraphs. Background and preevent images were subtracted from the event images, to remove undesired and dust-scattered light, as well as coronal structures not relevant to the CMEs under study. Images were processed according to the standard secchi prep procedure, and binned to $512 \times 512$ pixels to avoid errors due to delays between acquisition of images of the same polarization sequence. Total brightness $\left(T_{\mathrm{b}}\right)$, polarized brightness $\left(P_{\mathrm{b}}\right)$, unpolarized brightness $\left(U_{\mathrm{b}}\right)$, and polarization angle images were obtained for each snapshot of the studied CMEs.

The polarization technique employed for this analysis (Moran \& Davila 2004) makes use of $P_{\mathrm{b}}$ and $U_{\mathrm{b}}$ images calculated from a sequence of images polarized at three different angles. The pixel values in a ratio image $R_{\mathrm{m}}=P_{\mathrm{b}} / U_{\mathrm{b}}$ depend on the distance of the scattering element from the POS, being maximum when light is scattered from a point located on the POS. A proxy of the distance from the POS $|z|$ of a scattering point may thus be deduced through comparison with a set of theoretical $R t$ values, with $R t$ being a function of the point distance projected on the POS and its angle above the same plane. There is uncertainty in the sign of $z$, i.e., it is not straightforward to know whether the scattering point is in front of or behind the POS, unless the solar source of the scattering structure can be identified from the interpretation of, e.g., EUV images. In addition, it has to be kept in mind that the $|z|$ value corresponds to the average height of all scattering elements along the same line of sight.

The outcome of the technique applied to COR1 and COR2 images is visualized by means of topographical maps, which represent the average distance to the POS of the scattering structures. Only COR2 maps will be discussed here, since changes are specially evident across its larger FOV. Such maps, presented in Figures 18-20, are interpreted for each of the three events in the next subsections. The analysis is performed in the context of the respective solar source regions of the CMEs. The sources were unambiguously identified in low-coronal images from the STEREO/SECCHI EUVI telescopes, especially in the Fe XII line. Given that the origin of CMEs is associated to photospheric regions of opposite polarities (see e.g., Feynman \& Martin 1995), their geometrical characteristics were approximated by those of the neutral lines that separate both polarities. Therefore, it is possible to calculate location, inclination, and extension of the solar sources and to relate them with corresponding basic aspects of the CMEs. The three-dimensional direction of propagation of CMEs, compared to the source region location, yields deflections not only in the latitudinal, but also in the longitudinal direction. Likewise, the orientation of a CME main axis can be contrasted against the tilt of its source region neutral line, to test the general assumption that both tend to be aligned (Cremades \& Bothmer 2004). 


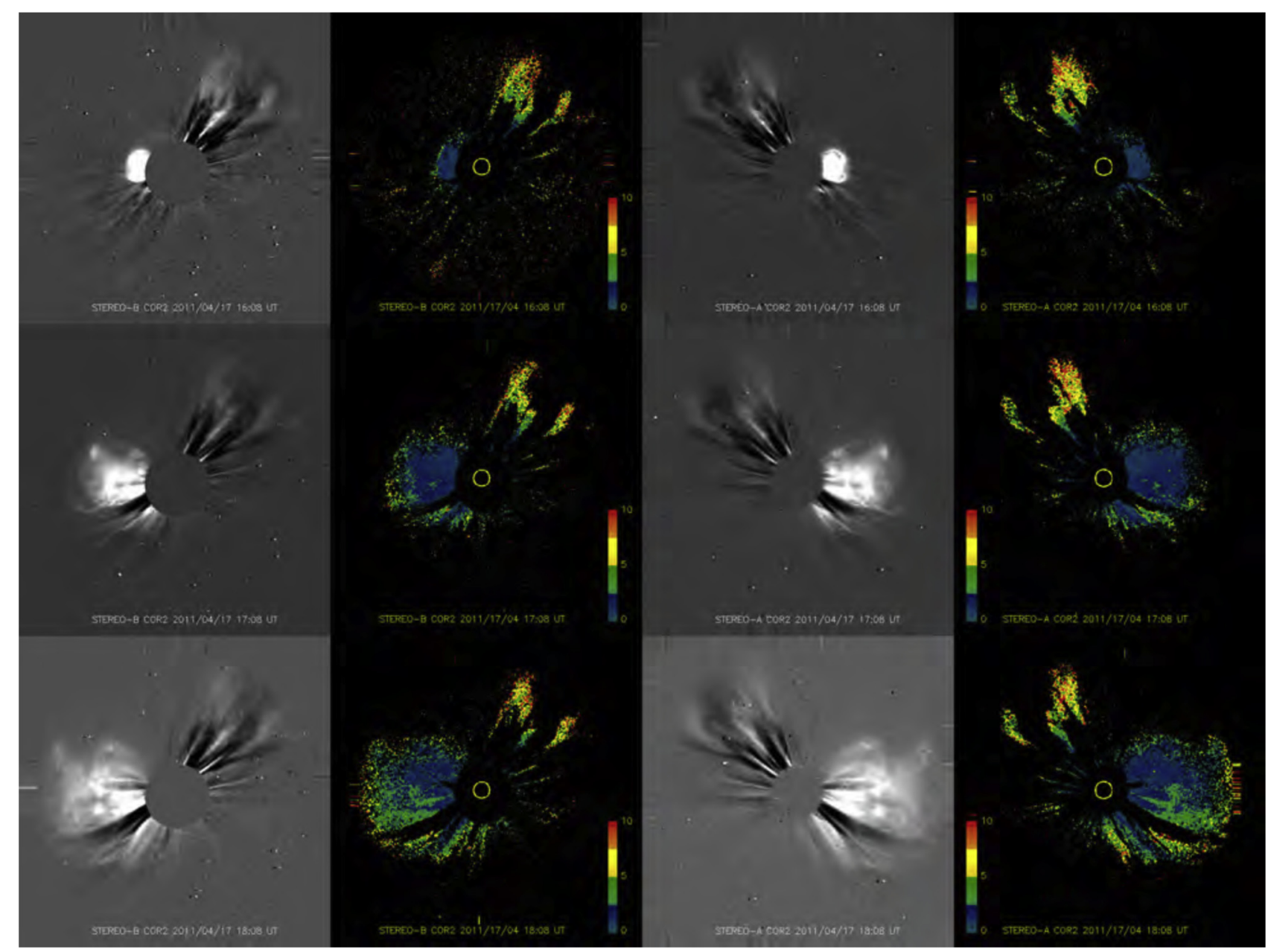

Fig. 19. Same as Figure 18 but for the event on April 17, 2011.

\subsubsection{The CME occurred on December 27, 2008}

On this date, the STEREO A and B spacecraft were approximately $90^{\circ}$ apart, and at the same time $\sim 45^{\circ}$ at each side of Earth (see first row of Fig. 17). The CME appears for the first time at 04:37 UT and at 10:08 UT in the FOVs of the COR2-A and COR2-B instruments, respectively. Inspection of EUVI images suggests that this $\mathrm{CME}$ arose as a product of a prominence eruption evident in the East limb of EUVI-A He II images (see Sect. 6). The same region observed with the Fe XII filter and from the perspective of EUVI-B has an "L" shape (see ellipse enclosing the region at Fig. 21a). The location of the source region (N40 E28 in heliographic coordinates) is consistent with the apparent shapes portrayed by the CME as viewed from different perspectives: in COR2-A a somewhat narrow loop, traveling approximately in COR2-A's POS, while in COR2-B a much wider, fan-like CME mainly propagating toward the COR2-B spacecraft. A first glance at Figure 18 confirms these statements: topographical maps of height above/ behind the POS indicate that the CME travels approximately in COR2-A's POS, given by the very low heights away from this plane, while analog maps from COR2-B show significantly more elevated heights, specially evident as the CME propagates outward from the Sun.

Once obtained height-time profiles both in the POS and in the $z$ direction of the CME's leading edge, it was possible to deduce its three-dimensional direction of propagation. The yielded value of $26^{\circ}$ in front of the POS in COR2-B's frame and $18^{\circ}$ behind COR2-A's POS, as compared to the central location of the solar source as viewed from EUVI-B and EUVI-A, respectively, indicate important deflections of $45^{\circ}$ and $37^{\circ}$ as independently calculated from each spacecraft's data. This yields an average longitudinal deflection of $41^{\circ}$ in the longitudinal direction, toward STEREO-B's POS. A similar analysis but performed in the latitudinal direction indicates an average deflection of $23^{\circ}$ toward the solar equator, when calculated both in the A and B spacecraft's frame. It has to be noted that the magnitude of the deflections needs to be understood in connection with the CME's angular width. Typical errors in $|z|$ measurements range from $\sim 1.5 \%$ to $\sim 3.0 \%$ (Lopez 2012).

The morphological analysis performed here makes use of the general scheme of three-dimensional CME configuration suggested by Cremades \& Bothmer (2004). The latter is in agreement with the findings of Moran \& Davila (2004) (i.e., consistent with the loop-arcade configuration) and drove Thernisien et al. (2006) to develop the now widely used Graduated Cylindrical Shell forward model. The scheme considers that CMEs can be approximated by a flux rope (as first suggested by Chen et al. 1997), which holds the symmetry of a cylinder. The orientation of the CME main symmetry axis is related to the tilt of the neutral line that separates opposite polarities within the source region. At the same time, the 


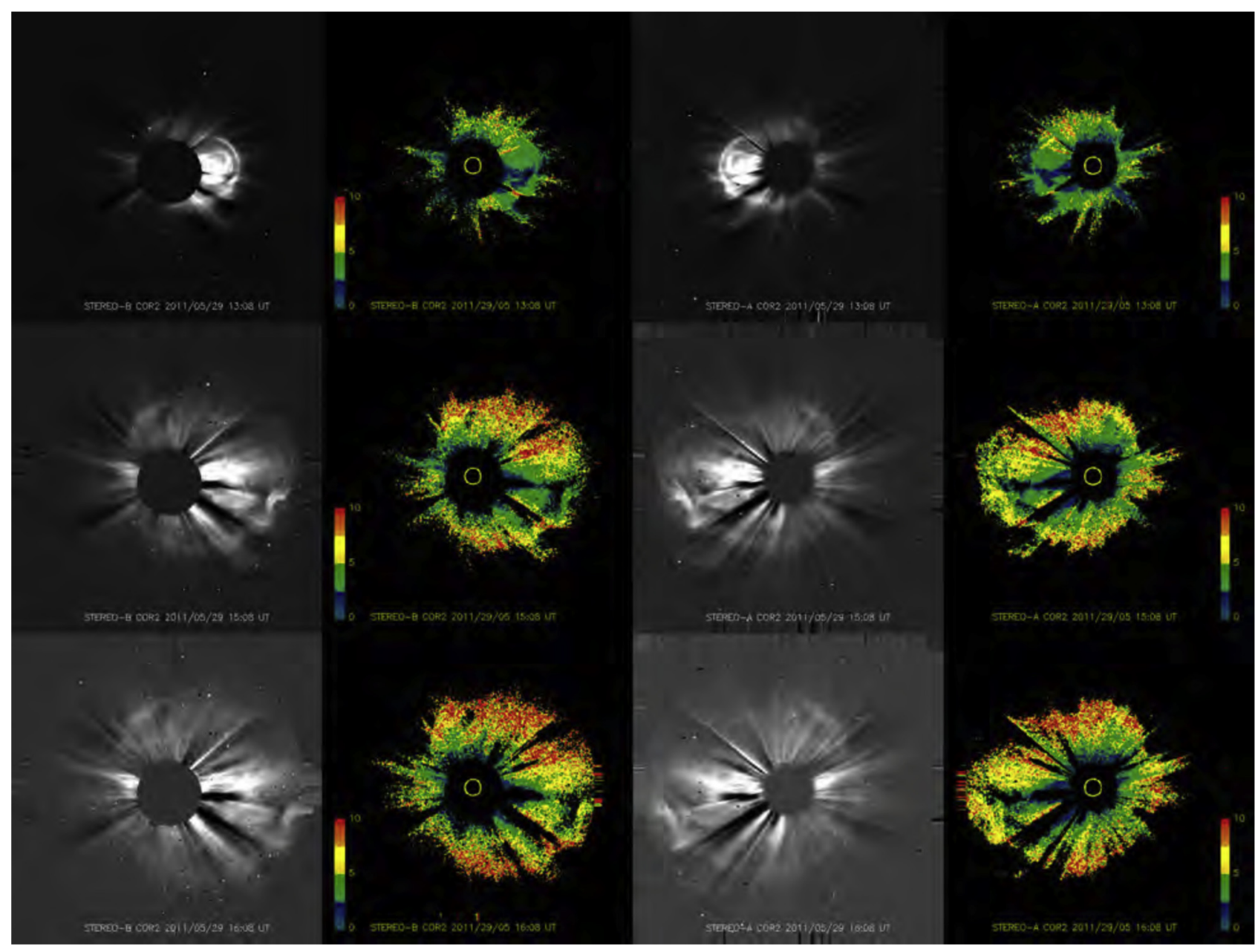

Fig. 20. Same as Figure 18 but for the event on May 29, 2011.

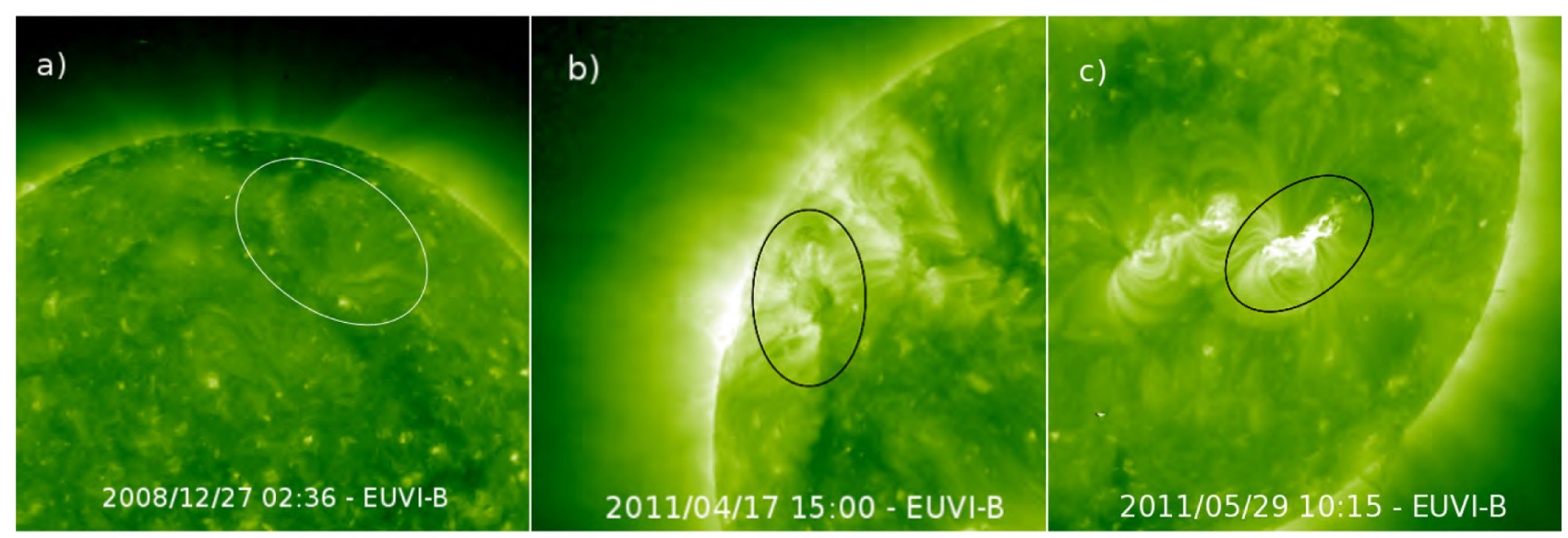

Fig. 21. EUVI-B images of the low corona in the Fe XII emission line, showing encircled the candidate sources of the three analyzed events. From left to right: (a) December 27, 2008, (b) April 17, 2011, and (c) May 29, 2011.

CME main axis is aligned with the cylinder's axis and the CME's bright core axis. Neutral lines nearly parallel to the limb would tend to be associated with side-view CMEs, while neutral lines perpendicular to the limb would be prone to yield front-view CMEs, i.e., with the typical three-part structure of a circular outer leading edge, a dark void, and a bright core.

In the case of this event, the irregular "L" shape exhibited by the neutral line complicates the direct understanding of the
CME configuration. To approximate this region by a single straight segment would lead to an oversimplification, thus the shape of the outer shell has to be regarded not as a cylinder, but as a deformed version of it. From STEREO-A's perspective, the prominence is observed erupting above the limb at a latitude that coincides with the horizontal (E-W) segment of the "L" neutral line. The CME, as observed by COR2-A, exhibits an apparent shape consistent with a view along the main axis, in 


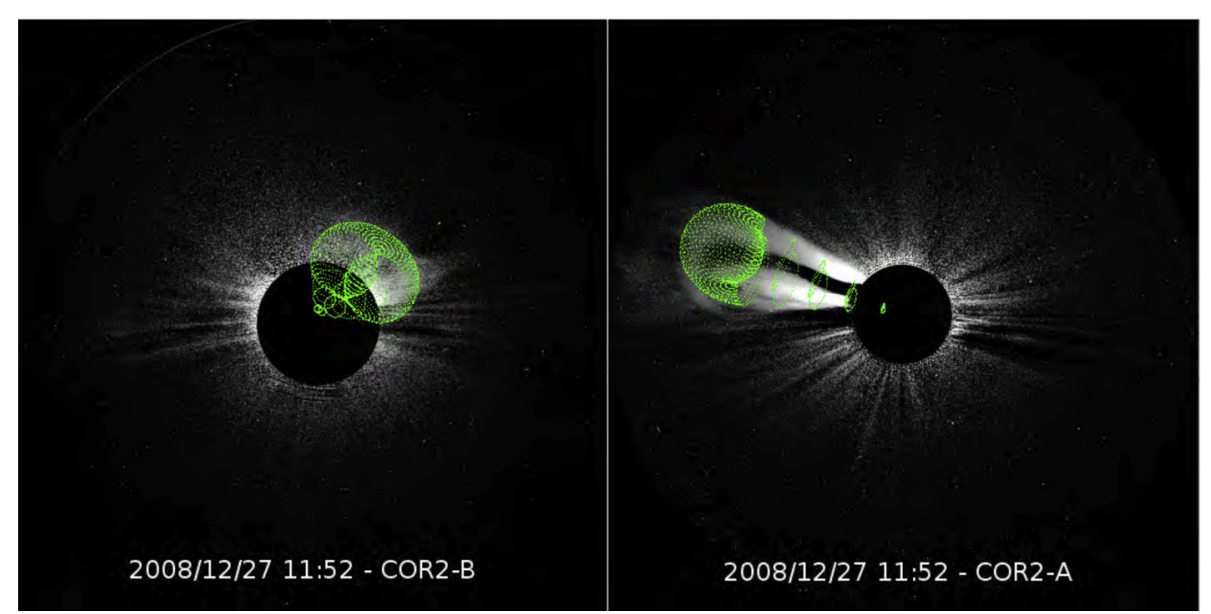

Fig. 22. Simultaneous fitting of a graduated cylindrical shell representing a flux rope CME, superimposed to total brightness images of two different views of the CME. Left: view from COR2-B, right: from COR2-A.

agreement with a front view of a CME with a neutral line perpendicular to the limb (see third column in Fig. 18). In addition, colors in the maps of height above/behind the POS indicate that the bulk of the CME is nearly traveling in STEREO-A's POS, with a small angle from it evident by the late increase in height of the CME leading edge (see fourth column in Fig. 18). From STEREO-B's perspective, the source region is not centered in the solar disk, but rather in the NW quadrant, at central heliographic coordinates N40 W17. Therefore, the CME's mass is mostly concentrated in a fan-like fashion in the NW region instead of producing a symmetric, full halo CME (first column in Fig. 18). The high propagation component in the direction of STEREO-B is evident in the second column of Figure 18, given the high gradient of heights in front of the POS seen throughout the CME, with the outer leading edge having reached larger heights. The fan-like shape is consistent with the side view of a cylindrical CME, also in agreement with the general scheme of CME configuration. To validate this reasoning, and to better illustrate the overall three-dimensional configuration of this first analyzed case, the outer shell of the CME has been simulated with the Graduated Cylindrical Shell forward model by Thernisien et al. (2006). Figure 22 depicts the same simulated 3D shape projected and superimposed to both views of the same CME. Note that this FM technique simulates "croissant-like" CMEs, though still in agreement with the concept of flux ropes and the simple cylinder. From Figure 22 it is clear how the same event may exhibit a completely different appearance depending on the observer's location.

\subsubsection{The CME occurred on April 17, 2011}

During the occurrence of this CME, the STEREO spacecraft were $\sim 180^{\circ}$ apart, and $\sim 90^{\circ}$ each from Earth. The fact that the CME was observed as a halo by $\mathrm{SOHO} / \mathrm{LASCO}$ indicates that this event would approximately propagate along the SunEarth line, and would have originated close to the limb as seen from the STEREO spacecraft (see second row of Fig. 17). The CME appears for the first time at 15:54 UT in the FOV of the COR2-A and COR2-B instruments. Investigation of low-coronal images from the EUVI instrument indicates that this event arose after the eruption of an unnumbered active region, visible close to the solar limb of EUVI-B (see Fig. 21b). It was not possible to observe this region in EUVI-A or EIT, given that it was located on their respective back-sides of the Sun. The neutral line corresponding to the identified source region may well be approximated by a linear segment, centered at N17 E55 (as seen from STEREO-B). The solar source location, as well as the CME direction of propagation evident in the COR2-A and COR2-B coronagraphs, indicate that the CME nearly propagates along the Sun-Earth line but away from Earth.

The direction of propagation of this event, as calculated from COR2-B polarized data, is $13^{\circ}$ in front of its plane of the sky. From COR2-A data the obtained value is $14^{\circ}$ but behind its POS. These results indicate a symmetrical scenario, consistent with a longitudinal deflection of $\sim 22^{\circ}$ toward the POS of both STEREO-A and B, i.e., toward the Sun-Earth line. This also explains the almost identical but flipped topographical maps (see second and fourth columns in Fig. 19), given that the bulk CME direction of propagation is at the same distance in front of/behind the POS for both spacecraft due to the $180^{\circ}$ separation. In the latitudinal direction, a similar analysis yields a deflection of $\sim 12^{\circ}$ toward the solar equator.

The neutral line associated to the solar source of this event is almost vertically oriented (N-S). The appearance of this $\mathrm{CME}$, as captured by the COR2-A and COR2-B coronagraphs, is comparable to a side view of a cylindrical CME (see first and third columns in Fig. 19), in direct accordance with the scheme of CME configuration (Cremades \& Bothmer 2004). Likewise, the highly centered full halo CME observed by SOHO/LASCO (see Fig. 17) exhibits an elongated outline mostly aligned with the N-S direction, also indicative of the orientation of the CME's main axis of symmetry.

\subsubsection{The CME occurred on May 29, 2011}

The CME on May 29, 2011 occurred when the STEREO spacecraft were $\sim 180^{\circ}$ apart. Since both STEREO A and B observed the CME as a full halo, it stands out that the CME must have been observed as a limb event from Earth's perspective (see third row of Fig. 17), i.e., it travels along SOHO's POS. The full halo CME appears for the first time in the COR2-A and COR2-B coronagraphs at 11:24 UT, while the limb loop-like CME appears in SOHO/LASCO's FOV at 10:36 UT. The candidate source of this event derived from EUVI low-coronal images is AR NOAA 11226, located in the SW quadrant of the solar disk from STEREO-B's perspective (see Fig. 21c) and backsided from STEREO-A. The neutral line at the base of this active region can be approximated by a straight line, 
which is tilted $\sim 50^{\circ}$ toward the direction of the N-S line. The source region location, at S21 W41 in EUVI-B, indicates that this CME has a strong component of propagation toward STEREO-B.

According to the results obtained from the polarization data, the denser and faster portion of this halo CME event (see first and third columns in Fig. 20) propagates in a direction $22^{\circ}$ degrees away from COR2-B's POS, and $35^{\circ}$ away from COR2-A's. These angles translate into an average deflection of $24^{\circ}$ in the longitudinal direction toward the STEREOs' POS, i.e., nearly toward the Sun-Earth line. However, the interpretation of this result needs caution, because it has been derived from a portion of the halo $\mathrm{CME}$ at equatorial latitudes and is not an average of all the halo CME extensions at all latitudes. In the latitudinal direction, the same denser and faster front appears deflected toward the solar equator by an amount of $\sim 20^{\circ}$.

The evolution of heights in front of the POS as depicted in the topographical maps of Fig. 20 (second and fourth columns) indicates that the northern halo CME extensions are at larger heights than the southern ones. The reason for this is plausibly a propagation direction slightly deviated toward the $\mathrm{S}$ direction. The evolution of the CME in the COR2 FOV evidences this situation: although in the first image of the sequence (top row in Fig. 20) the CME appears more extended toward the $N$, in the last one it looks mostly developed toward the $\mathrm{S}$ direction. The E-W asymmetry is most likely caused by the source of the CME being $\sim 40^{\circ}$ off-centered with respect to the STEREOs' views.

The post-eruptive loops associated to the solar source of this event appear with a tilt of $\sim 50^{\circ}$ from the horizontal. The CME main axis appears, however, to be almost horizontal, as also shown in the topographical maps (the halo CME regions at equatorial latitudes show lower $|z|$ values than the CME portions in the $\mathrm{N}$ and $\mathrm{S}$ directions). The circular arcs in the $\mathrm{W}$ portion of the halo CME in COR2-B are associated with one of the top cylinder surfaces, while the structures elongated and extended toward the $\mathrm{N}$ and $\mathrm{S}$ of the coronagraph's FOV would represent the loops that close on themselves constituting the body of the cylinder. COR2-A, being almost $180^{\circ}$ away from COR2-B, visualizes virtually the same structures.

The three-dimensional characterization of the CME morphology here presented yields results in agreement with the views of each CME from the perspective of each instrument, and at the same time in accordance with the scheme of CME configuration described above. Basic characteristics of the candidate source regions, like heliographic coordinates and neutral line tilt, were contrasted to the CMEs direction of propagation, main symmetry axis orientation, and general three-dimensional morphology. In all of the analyzed cases, significant deflections were found, not only in the latitudinal direction, but also in the usually unexplored longitudinal one (see Gui et al. 2011; and references herein). The performed morphological analysis revealed that the CMEs main axis orientations are in general accordance with their associated source region tilts. A notable agreement was found in the first two cases, and a clockwise rotation of $\sim 35^{\circ}$ is suggested in the third case. Based on the same line of thought, e.g., Thernisien et al. (2006) and Yurchyshyn (2008) reach comparable results through different analyses.

The additional view provided by the STEREO Mission contributes not only to validate the results obtained on the basis of single-spacecraft observations, but also to determine uniquely the direction of propagation and other three-dimensional properties. In addition, the small time delay between images of a same polarization sequence acquired by STEREO coronagraphs was essential to avoid errors due to the propagation of structures during the time of the observations. The agreement found in the results derived from different spacecraft (STEREO-A and STEREO-B) is encouraging. Ultimately, it may lead us to predict the characteristics of an incipient CME based on singlespacecraft data of its solar source and its impact on the Space Weather conditions.

\section{CME evolution from the solar atmosphere to the Earth: events occurred on April 3 and 8, 2010}

In this Section we describe the results from two case studies, on the April 3 and 8,2010, tracking the 3D structure of CMEs that arrived at the Earth and produced moderate geomagnetic storms. Both these events have been intensively studied (see, e.g., Möstl et al. 2010; Davis et al. 2011; Liu et al. 2011; Rouillard et al. 2011; Temmer et al. 2011; Wood et al. 2011) as they were the first ones during the STEREO era to induce geomagnetic disturbances and had a subsequent influence on technology (see, e.g., the possible temporary loss of communication with Galaxy 15 satellite - Connors et al. 2011). Here, we describe our results and compare them with those described in the literature listed above. Firstly, we describe the CME signatures observed in the lower corona, including: dimmings, solar flares, erupting filaments, and post-eruptive arcades. Then we apply the reconstruction methods, described in the previous Section to white-light observations, in order to determine the properties (e.g., speeds and direction of propagation) of the CMEs as they pass through the COR and HI fields of view. In situ data is used to derive the parameters of the CMEs at the spacecraft (temperature, speed, density, etc.). And geomagnetic data, through the $A p$ and Dst indices, are used to gauge the impact they had on the Earth's magnetic field.

\subsection{Source region and radio signatures}

On-disk, EUV images recorded by SWAP/PROBA2 and EUVI/ STEREO show EIT waves, EUV dimmings, as well as post-eruptive arcades. See Figure 23 for the location of the source region of the CME on April 3, 2010. Both CMEs were associated with flares (observed by LYRA/PROBA2) and erupting filaments. The EUV evolution of the active region associated with the April 3 event was presented in Seaton et al. (2011). Using SWAP and EUVI images Seaton et al. (2011) observed a cold plasma blob (part of the eruptive prominence) which was launched in the southward direction at around 08:18 UT, eventually destabilizing the filament through a mass off-loading process. Following this, a sudden expansion was observed at around 08:55 UT. This event was also studied by several other authors. Zuccarello et al. (2012c) concluded that both the magnetic configuration of the system and the photospheric dynamics that preceded the event were favorable for the eruption to occur, and Liu et al. (2011) showed that the CME onset took place earlier than the associated flare.

Several authors have also studied the second event on April 8,2010 , and detailed description of the source region can be found in Davis et al. (2011), Liu et al. (2010), Su et al. (2011), Ofman \& Thompson (2011). Su et al. (2011) concluded that this event may be initiated by a catastrophic loss of equilibrium caused by an increase of the axial flux in the flux rope, due 


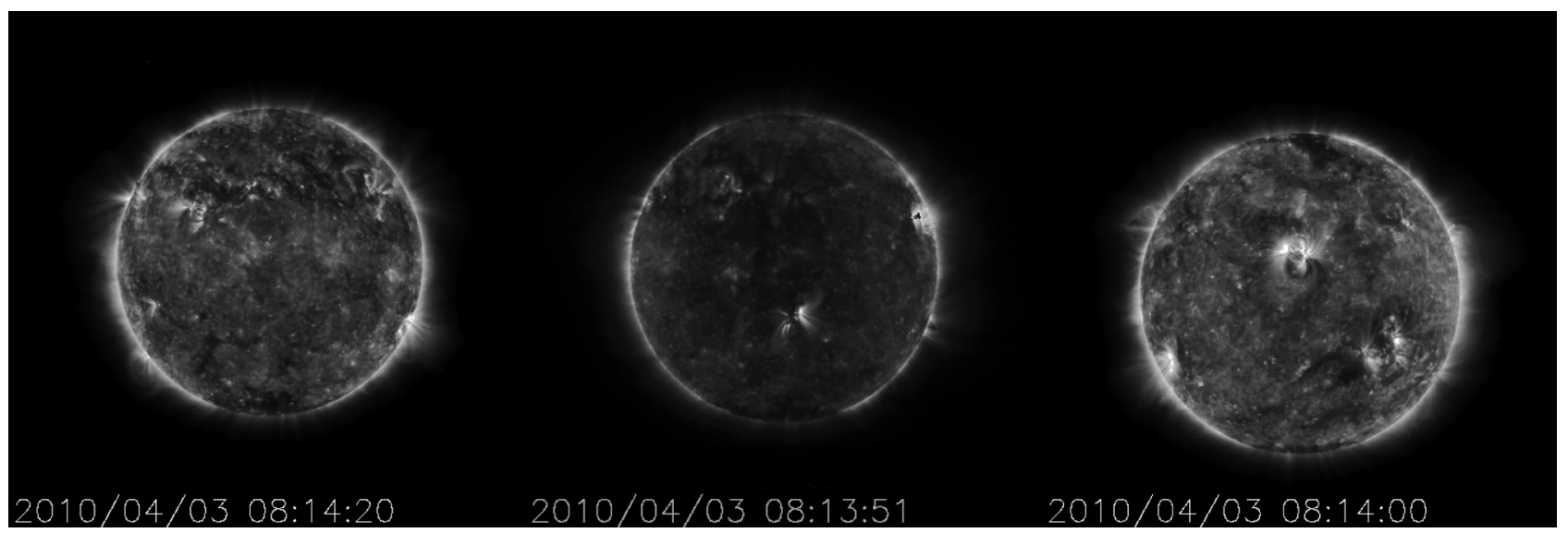

Fig. 23. Left panel: EUVI-B $17.1 \mathrm{~nm}$ image; middle panel: SWAP $17.4 \mathrm{~nm}$ image; right panel: EUVI-A $17.1 \mathrm{~nm}$ image. The images were recorded at around 08:14 UT. The active region is observed near disk center (slightly south) in the SWAP image, on the West limb in the EUVI-B image and on the East limb in the EUVI-A image.

to flux cancellations. Using data from SDO, Ofman \& Thompson (2011) showed that a Kelvin-Helmholtz instability was created by the velocity shear between the erupting and closed magnetic field of the coronal mass ejection. There were two filaments associated with this event: a small one along the polarity inversion line of the AR and a bigger one surrounding the AR were observed in SDO/AIA $304 \AA$ channel. Around 02:10 UT, material started to be ejected from the internal (small) filament and about 18 min later the filament began to lift off. This was followed by the first flare brightenings and the eruption of the surrounding (big) filament which was later seen as a CME (Su et al. 2011).

A radio III burst and radio drift signatures were associated with the April 3 event (see Fig. 24). A radio II burst was not observed at metric wavelengths although a CME-driven shock was observed (Liu et al. 2011). A km type II radio burst was detected by WIND/Waves (Bougeret et al. 2008) on April 4 from 00:58 until 16:33 UT (Xie et al. 2012). The associated shock arrived at the Wind spacecraft on April 5, around 08:00 UT. An interplanetary type III radio burst observed by WIND/Waves and STEREO/Waves A (Bougeret et al. 2008) at around 03:00 UT was associated with the April 8 event (Magdalenic, priv. commun.). A possible very weak and short type II radio burst (signatures of a shock wave) is observed in STEREO/Waves A instrument at about 03:40 UT. The shock arrived at ACE on April 11 around 12:14 UT.

\subsection{White-light signatures}

Two halo CMEs were observed by the SOHO/LASCO coronagraph on April 3 and 8, 2010. These events were observed as limb CMEs from the STEREO-B and -A spacecraft (138 separation angle). On April 3, 2010 a three-part CME was observed by the COR1 and COR2 instruments on board STEREO (see Fig. 25, upper panels). A partial halo CME was observed in LASCO C2 images (Fig. 25, lower left panel). As the CME propagated away from the Sun it passed into the HI1 and HI2 FOVs (Fig. 25, lower middle and right panels). The bright core of the CME is associated with the prominence material. Also, a diffuse bright feature is observed in the front of the leading edge (LE) of the CME, which may be the indication of the CME-driven shock. This diffuse feature is not well observed in the running-difference images shown in Figure 25 where instead, the LE is well visible as the bright circular feature seen ahead of the bright core. To better visualize the shock we compute a daily minimum background that we subtract from the frame with the CME. This background is created by taking the minimum value in each pixel, over the images of the period when the CME was observed (see also Mierla et al. 2009). The period is different depending on the number of frames the CME is seen: e.g., for COR1 the images are recorded during $5 \mathrm{~h}$ while for COR2 the images are recorded during 11-13 $\mathrm{h}$.

The April $8 \mathrm{CME}$ was also observed as a limb event in STEREO COR images, with a similar configuration to the CME observed on April 3. However, the LASCO observations yielded a halo CME. Through the combination of observations outlined above, we were able to track both CMEs from the lower solar atmosphere all the way to the Earth.

\subsection{D reconstruction results}

\subsection{1. $F M$}

By applying the graduated cylindrical shell model described above to the COR data (see Fig. 26) we were able to estimate several characteristics of the CME. These are displayed in Table 1. Parameters of the CMEs on April 3 and 8, 2010, as derived from FM method. First and second columns: the date and time when the CME was observed in COR data. Columns three and four: the heliographic longitude and latitude. Column five: the maximum distance of the front side CME surface from the Sun center. Column six: orientation of the neutral line on the solar disk. Column seven: ratio of minor torus radius to the distance from the Sun center. Column eight: angular width between the legs of the model.

Both April 3 and 8 CMEs values are calculated, at six different times in COR1 and COR2 images. The speeds derived from the height parameters in time for the April 3 event are around $850 \mathrm{~km} \mathrm{~s}^{-1}$ with a final value of $876 \mathrm{~km} \mathrm{~s}^{-1}$ measured in COR2, at around 12:08 UT, and at an altitude of 13.82 solar radii. The speed measured by Kilpua et al. (2012) in COR2 FOV is around $700 \mathrm{~km} \mathrm{~s}^{-1}$, smaller than the speeds derived here because of the different features that were tracked (shock in this study and LE for Kilpua et al. 2012).

For the April 8 event the speeds vary from around $210 \mathrm{~km} \mathrm{~s}^{-1}$ (COR1, 3:55 UT, 2.70 solar radii) to $576 \mathrm{~km} \mathrm{~s}^{-1}$ (COR2, 07:08 UT, 11.85 solar radii). Again, the speeds 

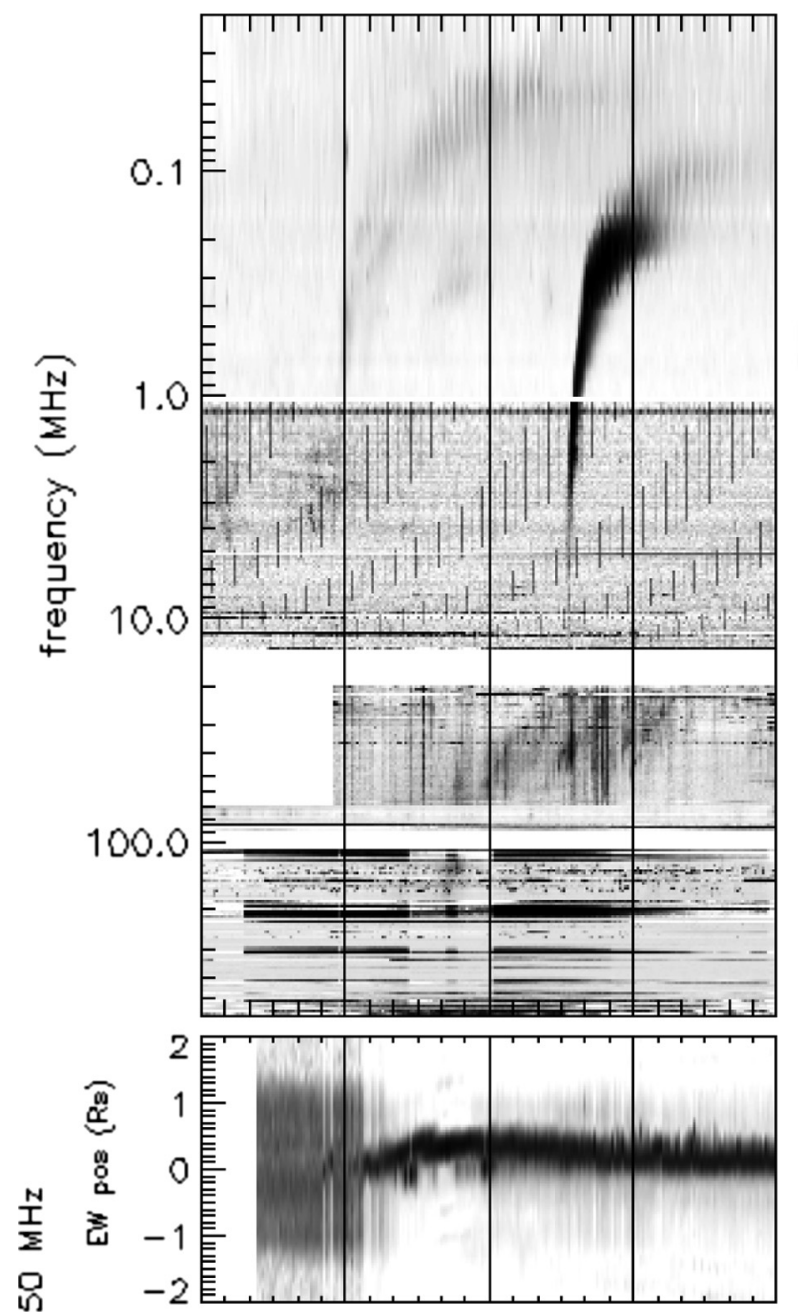

$\frac{1}{5}$

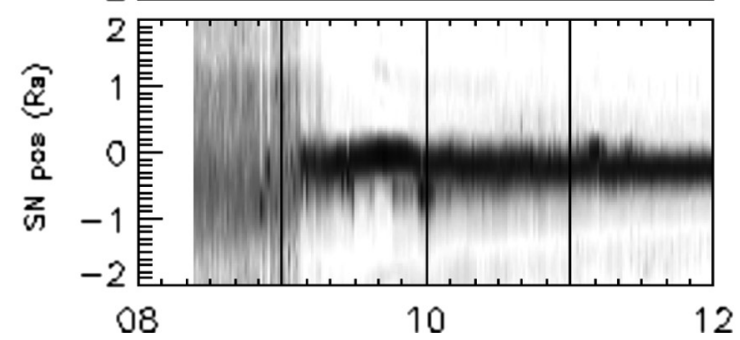

Fig. 24. The radio emission in the frequency range $20 \mathrm{kHz}$ to $600 \mathrm{MHz}$, and the time interval 08-12 UT, recorded on April 3, 2012. The composite dynamic spectrum shows the ARTEMIS (70$600 \mathrm{MHz})$, DAM $(20-70 \mathrm{MHz})$, and WIND Waves $(20 \mathrm{kHz}$ to $13.8 \mathrm{MHz}$ ) observations. The two lowest panels show the observations by the Nancay radioheliograph, at $150 \mathrm{MHz}$.

measured using the same method on COR2 data but for the LE by Kilpua et al. (2012) are around $576 \mathrm{~km} \mathrm{~s}^{-1}$, slightly smaller than the shock speed measured here. We have seen that the same method applied on the same event by different people may lead to different results. There are several explanations to this: (1) different features of the CME are tracked (shock or LE); (2) The CME does not exhibit a circular front characteristic to the model applied here (see, e.g., the case of April $3 \mathrm{CME}$ which is flattened and shows a dimple in its front, presumably owing to the distortion by the pre-existing heliospheric plasma sheet - Liu et al. 2011). This is further confirmed by Wood et al. (2011) which by using an empirical $3 \mathrm{D}$ reconstruction technique on the same event demonstrated that the CME can be reproduced reasonably well at all times with a 3D flux-rope shape, but the case for a flux rope being the correct interpretation is not as strong as other events studied with STEREO in the past.

\subsection{2. $P R$}

An example of reconstruction using PR method on COR1-A and COR1-B images recorded on April 3, 2010, 09:50 UT, is shown in Figure 27. The $X$-axis points toward the Earth and $Z$ toward the solar North. The gray sphere has a radius of 1 solar radii and the grid sphere has a radius of 1.5 solar radii. Blue points are the closest to the Earth and the red ones are the farthest from the Earth. The elongated feature along $Y$-axis represents a low polarized feature, which we think is the prominence which emits in $\mathrm{H} \alpha$ (Mierla et al. 2011). As both CMEs analyzed here are associated with prominence eruptions, there is a high probability that $\mathrm{H} \alpha$ emission is observed from the core of the CME. In this case the results derived using this method will yield unrealistic values and one should isolate these parts of the CME when deriving the true speeds and direction of propagation.

\subsubsection{Triangulation}

We apply the method described in reference Mierla et al. (2008) on a feature identified on the LE of the April 3 event (the dimple in its front). This feature was tracked in time in COR1 images and the reconstructed results are shown in Figure 28. The direction of propagation of the CME is the same (within the error range) as the one derived from FM technique: i.e., the location derived from this method is around $4^{\circ}$ for longitude and $-29^{\circ}$ for latitude (coordinates measured in HEEQ system) and from FM is $9^{\circ}$ for longitude and $-26^{\circ}$ for latitude. The mean value for the speed derived from HT diagram is around $140 \mathrm{~km} \mathrm{~s}^{-1}$. Liu et al. (2011) applied triangulation method on the CME front observed in running-difference time-elongation maps $(\mathrm{COR} 2+\mathrm{HI})$ and they show that speed first rises from less than $400 \mathrm{~km} \mathrm{~s}^{-1}$ to $1000-1100 \mathrm{~km} \mathrm{~s}^{-1}$ and then decreases to about $800 \mathrm{~km} \mathrm{~s}^{-1}$, presumably due to interactions with the background solar wind. The propagation direction derived from this method shows an indication of transition from eastward to westward at the early stage. After that, the propagation direction stays around $10^{\circ}$ west of the Sun-Earth line. Note that their feature is at the flank of the LE as compared with our feature which is more in the middle of the LE. The speeds cannot be compared directly, but it is clear that the CME undergoes a fast acceleration in COR1 and COR2 FOVs, with the acceleration continuing also after the maximum phase of the associated flare.

\subsubsection{Fixed- $\Phi$ and Point-P methods}

The LE was further followed out into interplanetary space, with HI1 and HI2 observations. This was done using the Fixed- $\Phi$ and Point-P methods described above. The height-time plots produced using these methods are shown in Figure 29. The feature we follow lies close to the ecliptic plane. We note that the points derived from the two methods diverge with increasing height through the HI2 FOV. This is because the CME has become an extended feature and the Fixed- $\Phi$ method works best on compact structures (Kahler \& Webb 2007). The speeds vary from $1055 \mathrm{~km} \mathrm{~s}^{-1}$ (at $20 \mathrm{Rs}$ ) to around $510 \mathrm{~km} \mathrm{~s}^{-1}$ (232 $\mathrm{km} \mathrm{s}^{-1}$ from Point-P method) close to the Earth. The speed of the ICME shock recorded at ACE is around 


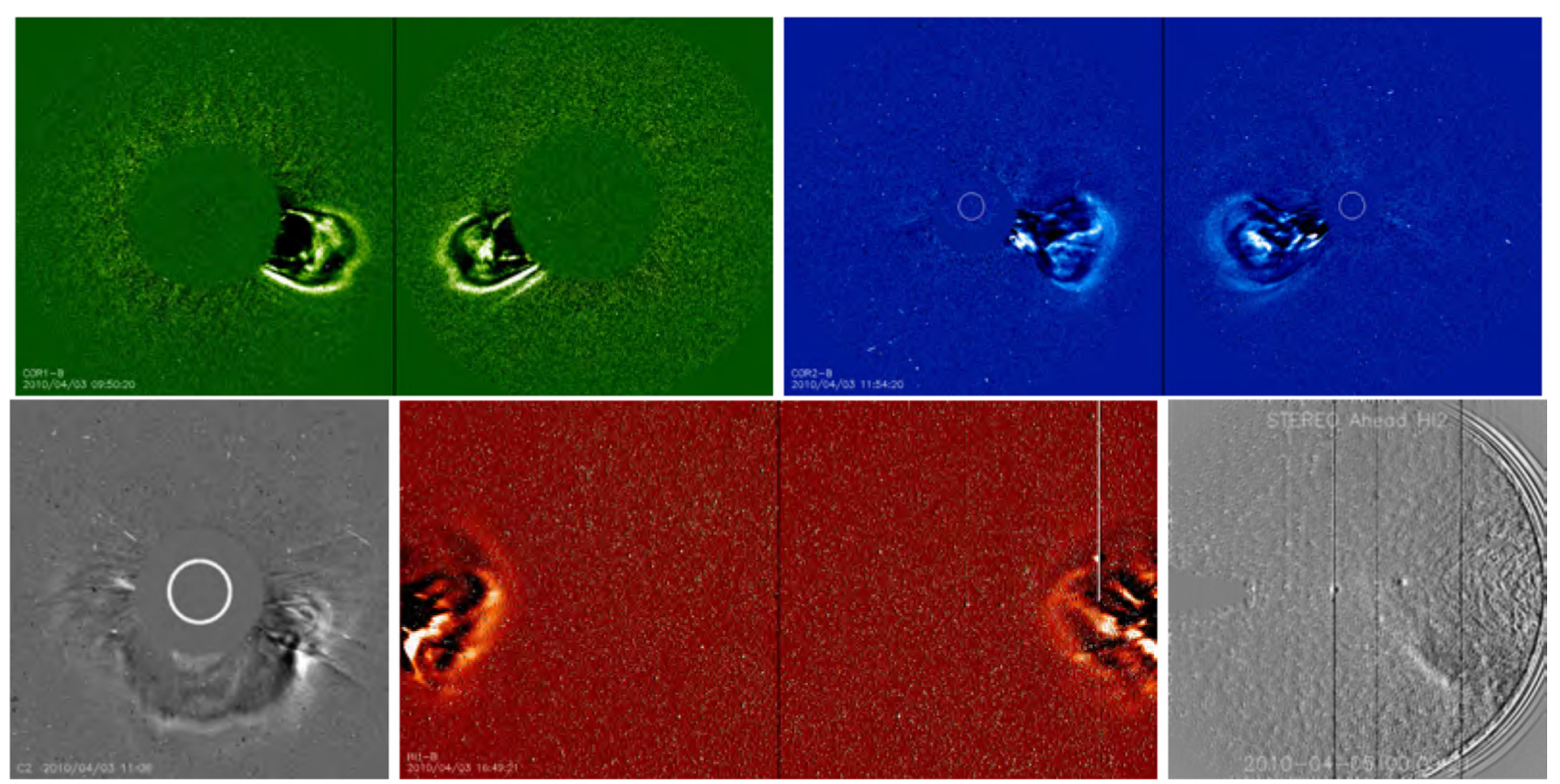

Fig. 25. COR1 images (upper left panel), COR2 images (upper right panel), LASCO-C2 (lower left panel), HI1 (lower middle panel), and HI2A (lower right panel) recorded on April 3, 2010, 09:50 UT (COR1), 11:54 UT (COR2), 11:06 UT (LASCO-C2), 16:49 UT (HI1), and April 5, 00:09 UT (HI2-A).
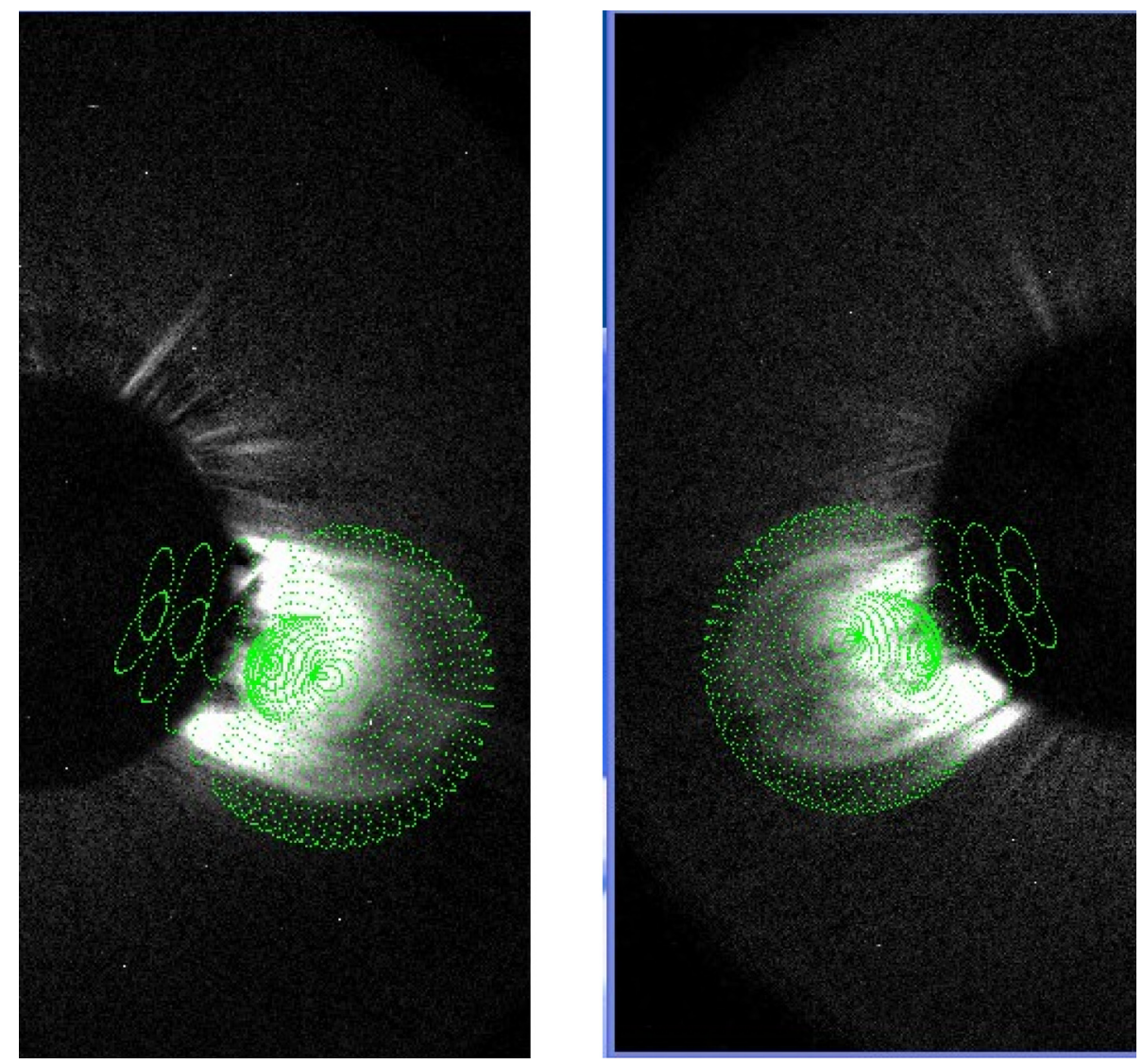

Fig. 26. Fitting of flux rope-like model to the COR1 data (A - right, B - left) on April 3, 2010, 09:50 UT. Note that compared with other studies we are fitting the diffuse bright structure in front of the LE and not the LE marked by the circular bright edge.

$800 \mathrm{~km} \mathrm{~s}^{-1}$. The average speed of the ICME was $650 \mathrm{~km} \mathrm{~s}^{-1}$ (Möstl et al. 2010).

Using Fixed- $\Phi$ method for a feature on the LE, with direction $9^{\circ}$ with respect to the Earth and Harmonic Mean method for a $\mathrm{LE}$ feature at $-5^{\circ}$ orientation with respect to the Earth Möstl et al. (2010) derived speeds of around $950 \pm 150 \mathrm{~km} \mathrm{~s}^{-1}$ at $0.1 \mathrm{AU}$ to $800 \pm 250 \mathrm{~km} \mathrm{~s}^{-1}$ near Earth. Their velocities are consistent with the maximum radial speed of $990 \mathrm{~km} \mathrm{~s}^{-1}$ 
Table 1. Note that the values shown here are slightly different from the ones in Kilpua et al. (2012), because in this study we used only two spacecraft, while in Kilpua et al. (2012), the SOHO spacecraft was used as the third eye on the Sun. Note also that the outer diffuse part of the CME was fitted by the model (see Fig. 26) - this should not be confused with the LE but with the shock of the CME. The shock is better visible in HI1 FOV as a sharp edge all the way around the CME front (see, e.g., Liu et al. 2011).

\begin{tabular}{lccccccc}
\hline \hline Date & Time (UT) & Longitude (HEEQ) & Latitude (HEEQ) & Outer shell height & Tilt angle & Ratio & Angular width \\
\hline April 3 (COR1) & $09: 40$ & 2 & -25 & 2.90 & 90 & 0.27 \\
April 3 (COR1) & $09: 45$ & 11 & -26 & 3.28 & 90 & 0.29 \\
April 3 (COR1) & $09: 50$ & 16 & -27 & 3.87 & 90 & 0.29 \\
April 3 (COR2) & $10: 08$ & 6 & -24 & 4.92 & 90 & 0.29 \\
April 3 (COR2) & $11: 08$ & 5 & -25 & 9.29 & 90 & 0.29 \\
April 3 (COR2) & $12: 08$ & 5 & -24 & 13.82 & 90 & 0.26 \\
April 8 (COR1) & $03: 45$ & -14 & -3 & 2.52 & -6 & 0.30 \\
April 8 (COR1) & $03: 55$ & -8 & -5 & 2.70 & -6 & 0.30 \\
April 8 (COR1) & $04: 05$ & -15 & -4 & 2.92 & -6 & 0.30 \\
April 8 (COR2) & $05: 08$ & -12 & -7 & 8.09 & -6 & 0.33 \\
April 8 (COR2) & $06: 08$ & 3 & -7 & 11.85 & -6 & 0.33 \\
April 8 (COR2) & $07: 08$ & 11 & & & -6 & 0.33 & 12 \\
\hline
\end{tabular}
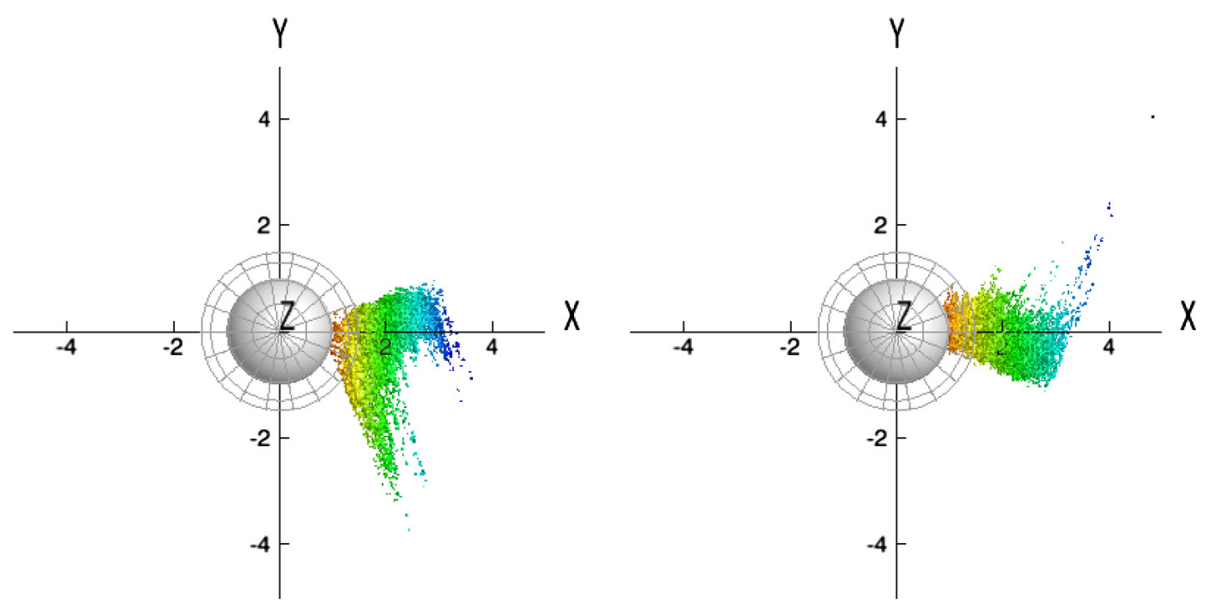

Fig. 27. A PR reconstruction of the CME observed in COR1 data on April 3, 2010, 09:50 UT. The view is from solar north. The left panel represents the reconstruction applied on COR1-B data and the right panel represents the reconstruction applied on COR1-A data. Note that due to the front-to-back ambiguity of the polarization, the reconstructed points are placed in front of the plane-of-the-sky of each spacecraft.

obtained with the stereoscopic FM method at distances closer to the Sun and with the in situ values recorded at Wind. The speeds derived by Liu et al. (2011) from triangulation method on HI data are around $800 \mathrm{~km} \mathrm{~s}^{-1}$ at distances close to the Earth. We see that the speeds we estimated from HI images are smaller than the speeds recorded at ACE and smaller than the speeds derived by Möstl et al. (2010) using the same method (i.e., the Fixed- $\Phi$ method). The explanation for these discrepancies is: different features are followed in HI images by different authors and/or the assumptions made in the two methods do not hold in this case. It is also important to select the same feature which is seen by different instruments on different spacecraft. As noted by Möstl et al. (2010), the LE of the ICME observed in HI time-elongation J plot maps was about $4 \mathrm{~h}$ too early at the elongation of Wind when compared with the shock arrival time on April 5, 08:00 UT. The authors explained this by a geometric effect, as the observer does not look exactly along LE when it hit WIND. Also, it is not clear which is the corresponding part of the LE observed by $\mathrm{HI}$ in the data from ACE or WIND. As described by Rouillard et al. (2011), different regions are observed by ACE for this event: the shock arriving at 08:00 UT on April 5, followed by the sheath region which is immediately followed by a less turbulent magnetic field region which the authors associated with the passage of the white-light driver gas, and finally, the

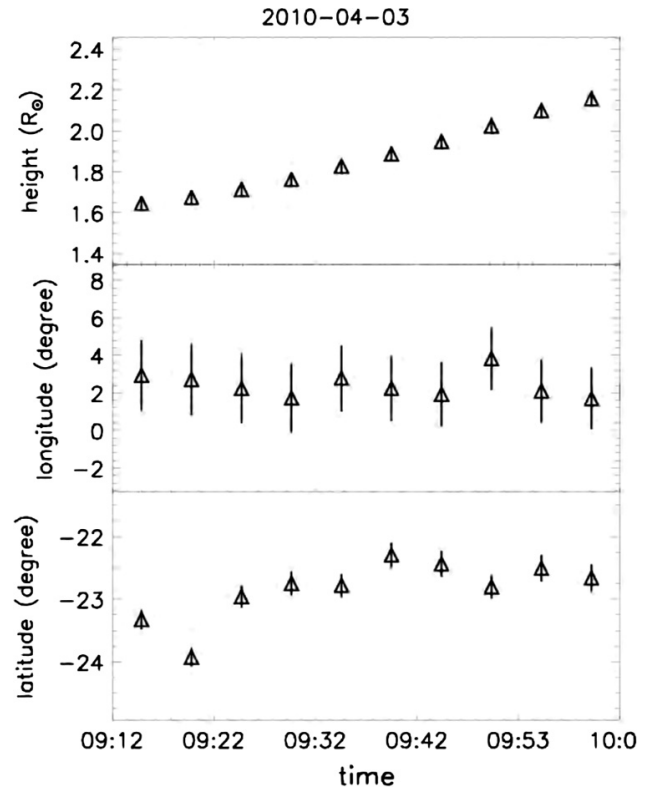

Fig. 28. Plots of height (upper panel), ecliptic longitude (middle panel), and ecliptic latitude (lower panel) versus time, for the identified feature on the LE of April 3, 2010 CME. The errors are calculated from the initially estimated errors in the radial direction $\left(0.038\right.$ solar radii) and in the azimuthal direction $\left(1^{\circ}\right)$. 

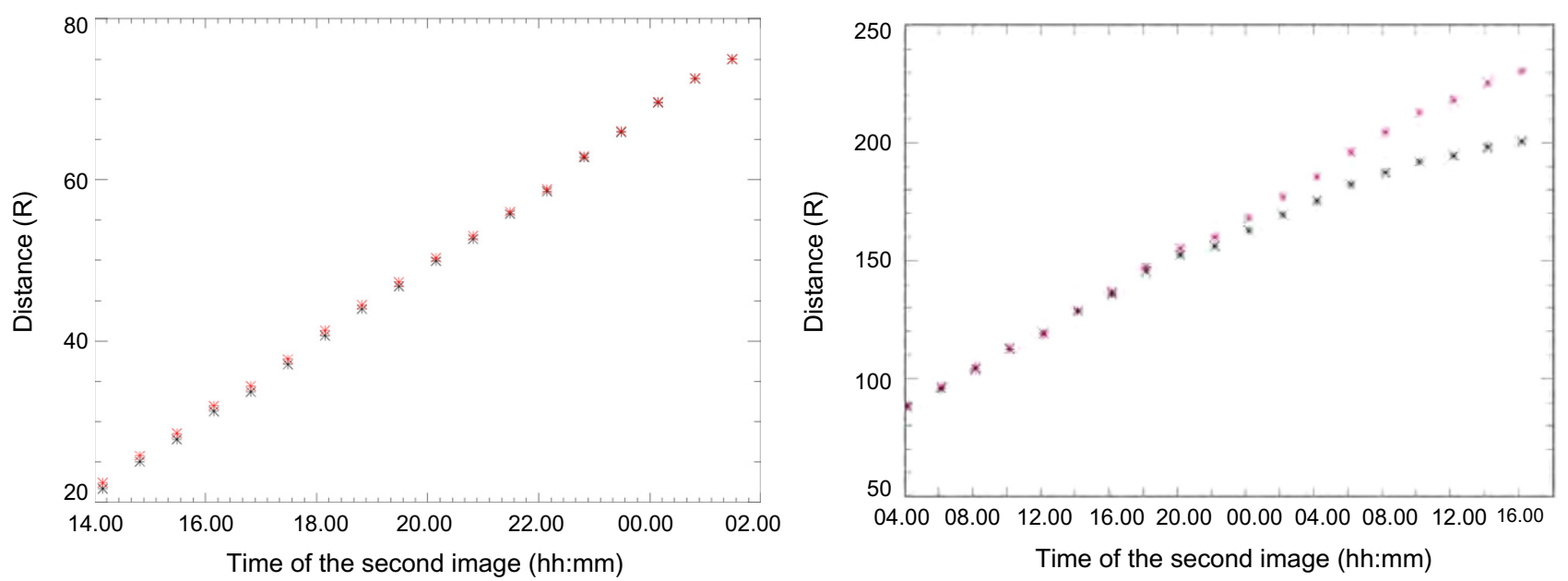

Fig. 29. Height-time diagrams for HI1-A (left) and HI2-A (right) by using the Fixed- $\Phi$ method (red symbols) and Point-P method (black symbols). The time varies from 14:00 UT (April 3) to 02:00 UT (April 4) for HI1-A, and from 04:00 UT (April 4) to 16:00 UT (April 5) for HI2-A.

arrival of the filament material toward the end of the passage of the driver gas. Note the name of driver gas instead of a magnetic cloud, as a clear flux-rope structure could not be identified with this event (Wood et al., 2011; Möstl et al. 2010).

The CME on April 8, 2010 was analyzed using the same methods (Point-P and Fixed- $\Phi$ ) in HI FOVs. The values obtained for the speeds are ranging from around: HI1-A: $75 \mathrm{~km} \mathrm{~s}^{-1}$ (at $19 \mathrm{Rs}$ ) to $510 \mathrm{~km} \mathrm{~s}^{-1}$ (at $70 \mathrm{Rs}$ ); HI1-B: $400 \mathrm{~km} \mathrm{~s}^{-1}$ (at $17 \mathrm{Rs}$ ) to $550 \mathrm{~km} \mathrm{~s}^{-1}$ (at $70 \mathrm{Rs}$ ); HI2-A: $280 \mathrm{~km} \mathrm{~s}^{-1}$ (at $87 \mathrm{Rs}$ ) to $160 \mathrm{~km} \mathrm{~s}^{-1}$ (at $149 \mathrm{Rs}$ ); HI2-B: $140 \mathrm{~km} \mathrm{~s}^{-1}$ (at $77 \mathrm{Rs}$ ) to $570 \mathrm{~km} \mathrm{~s}^{-1}$ (at $125 \mathrm{Rs}$ ). There is now a bigger discrepancy for the speeds near the Sun (750 $\mathrm{km} \mathrm{s}^{-1}$ for HI1-A compared with $400 \mathrm{~km} \mathrm{~s}^{-1}$ for HI1B) derived from the two spacecraft, compared with the April 3, 2010 event. We do not know what may be the cause for this, the only difference between the two events being the different location of the source regions (S25 W03 for April 3 event and N25 E16 for April 8 event).

As with the April 3 event, because the STEREO spacecraft were separated by $138^{\circ}$, a position angle of $90^{\circ}$ in A will correspond approximately to $270^{\circ}$ in $\mathrm{B}$ spacecraft. The features on the LE were tracked at position angles of: $94^{\circ}$ (HI1-A), $104^{\circ}$ (HI2-A), $267^{\circ}$ (HI1-B), and 275 ${ }^{\circ}$ (HI2-B).

\subsection{In situ signatures}

The ICME corresponding to April 3, 2010 event was observed in situ by ACE on April 5, 2010 (see Fig. 30). The shock arrives at 07:56 UT on 5 April and is visible by the sudden change in the plasma and magnetic field parameters (speed, density, temperature, and magnetic field magnitude). Liu et al. (2011) showed, by linking imaging observations with in situ signatures, that the sharp white-light feature observed in HI data is likely the CME-driven shock observed at ACE. Right after the shock, there is a sheath region with increased density and temperature and varying magnetic field, lasting until 12:00 UT. From here on, a rotation in the magnetic field components is present, showing the arrival of a magnetic cloud (MC). Nevertheless, the flux-rope-like configuration of the MC is not very clear. According to Möstl et al. (2010), the MC fulfills the Burlaga et al. (1981) criteria of low solar wind proton temperature, low plasma- $\beta$, and a smoothly rotating and higher-than-average total magnetic field, but it could not be fitted using the force-free model (Lepping et al. 1990) or reconstructed with the Grad-Shafranov technique ( $\mathrm{Hu} \&$ Sonnerup 2002). According to the low temperature and plasma- $\beta$, the ICME extends until 14:00 UT on April 6. A fast solar wind stream comes right after the ICME.

The April $8 \mathrm{CME}$ is visible at ACE on April 11. At 12:15 UT the shock arrives followed by a sheath region lasting until 21:00 UT, where the ICME material can be seen to start. There is a (not smooth) rotation in the field indicating a MC. The ICME signatures seem to last until 16:00 UT on April 12. As stated by Davis et al. (2011) a large and smooth rotation of the magnetic field is absent, so this structure is not likely to be a magnetic cloud with a typical flux-rope structure.

\subsection{Geomagnetic storms}

A moderate geomagnetic storm started at 9:00 UT on April 5, 2010. The Dst (hourly averages values) and Ap (3-hourly averages values) geomagnetic indices associated with the storm are shown in Figure 31. The minimum Dst $(-77 \mathrm{nT})$ is observed on April 6, around 15:00 UT. The geomagnetic storm started one hour after the shock was observed at ACE. The geomagnetic storm produced by the April $8 \mathrm{CME}$ started around 15:00 UT on April 11 and it had a maximum (Dst around $-66 \mathrm{nT}$ ) on April 12 at 02:00 UT. The geomagnetic storm started $3 \mathrm{~h}$ after the shock was observed at ACE. This is consistent with the smaller speed of the second CME compared with the first one. It is not possible to identify exactly the end of the first geomagnetic storm (starting on April 5), as during the period of magnetic field recovery, the second storm begins, as well as other smaller perturbations (see Fig. 31). It is noticed that the storm produced by the first CME is stronger than the second one.

\subsection{Travel time to the Earth}

We now compare our estimates of the time taken for the CMEs to travel to the Earth with the observed travel times. We do not use the values derived from PR method as we think that data were contaminated with $\mathrm{H} \alpha$ emission and the speeds will not 
F. Zuccarello et al.: Solar activity across the corona

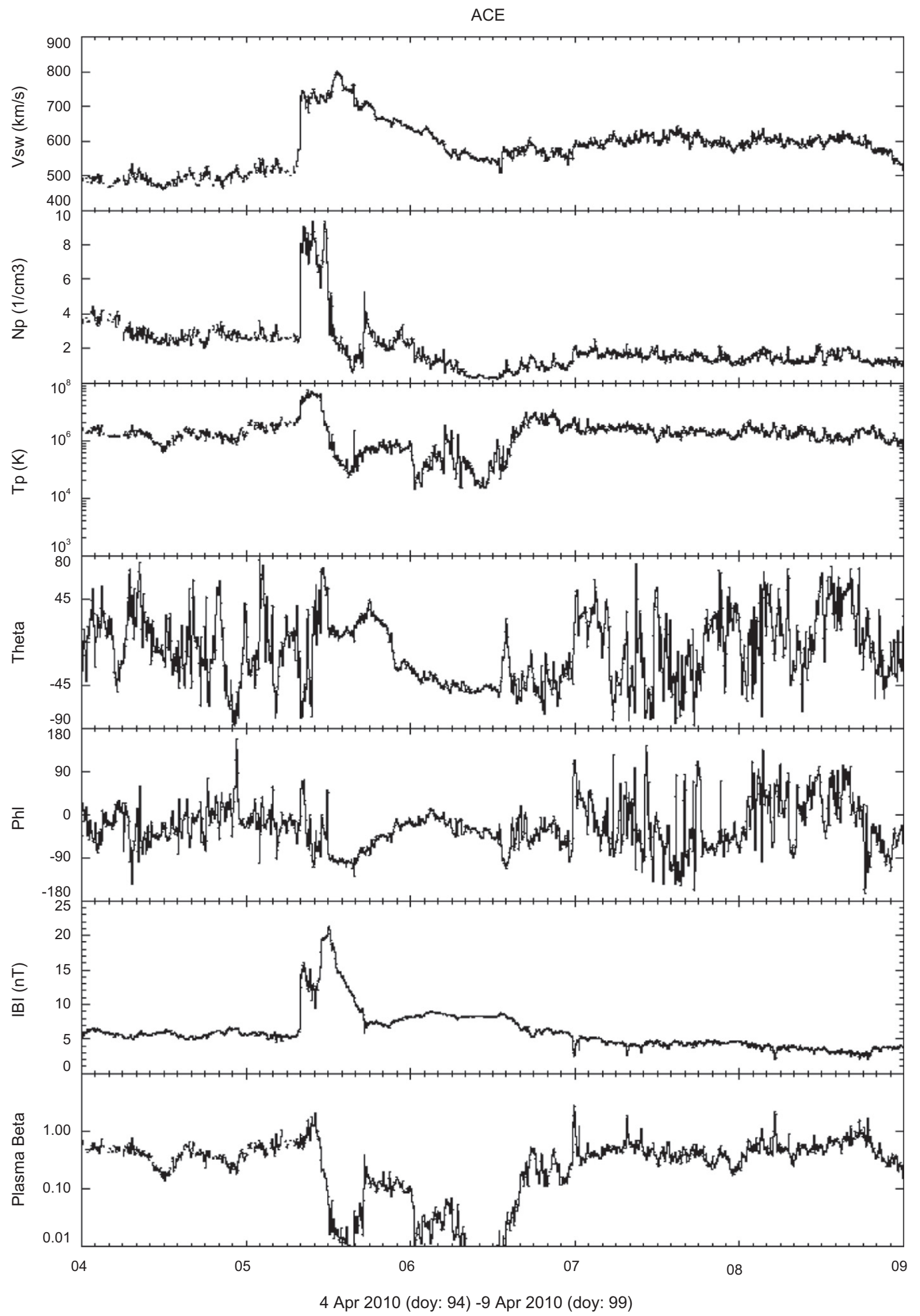

Fig. 30. ICME signatures for the April 3 event arriving at ACE on April 5, 8:00 UT. The panels from top to bottom are: solar wind speed, proton density, proton temperature, latitudinal angle of the magnetic field, azimuthal angle of the magnetic field, magnitude of the magnetic field, and plasma- $\beta$.

reflect the real speeds of the bulk material moving toward the observer. We use instead the speeds derived from FM which give us the values of the shock speeds close to the Sun. Note that compared with similar studies (Möstl et al. 2010) on the same events we derive the speed of the shock and not of the LE. 

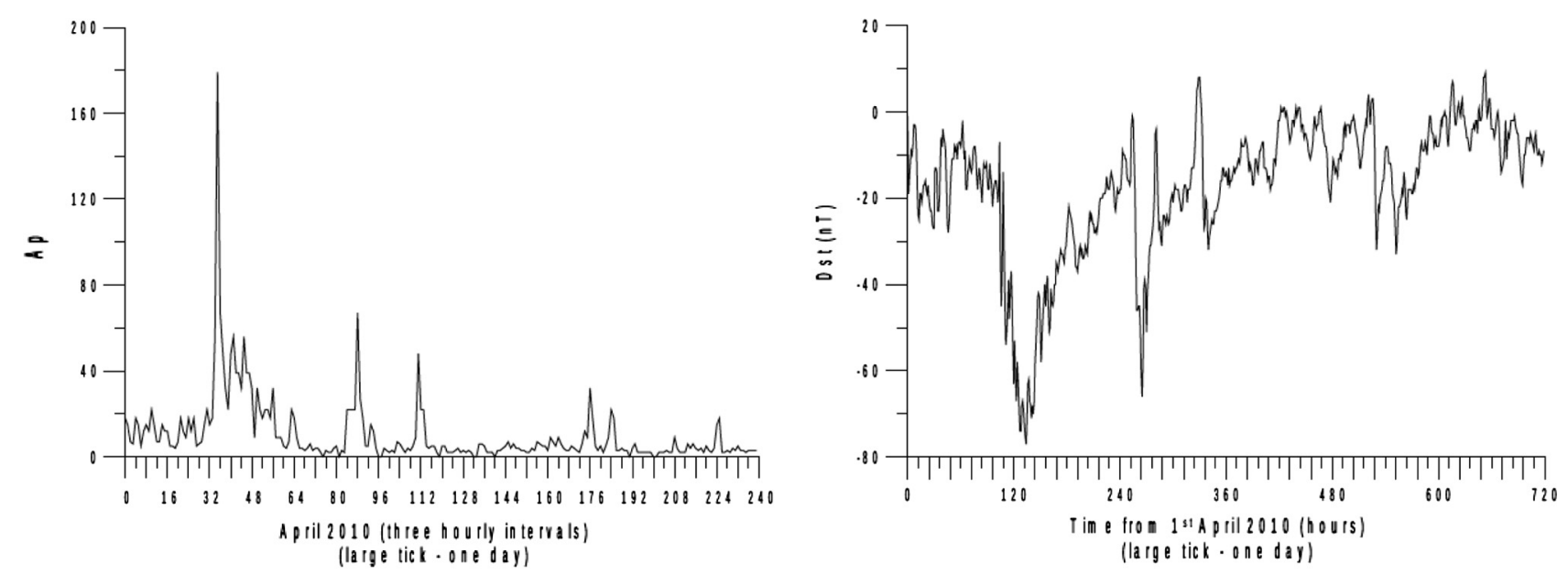

Fig. 31. Three-Hourly average values for Ap index (left panel) and hourly average values for Dst index (right panel) for April 2010. Courtesy of V. Dobrica.

\subsubsection{April 3, 2010}

If we take the final speed $\left(876 \mathrm{~km} \mathrm{~s}^{-1}\right)$ observed in the COR2 FOV, derived using the FM technique, as being the speed the CME propagates into the interplanetary space, we obtain a travel time to the Earth of $44 \mathrm{~h} 31 \mathrm{~min}$, while the real travel time was $43 \mathrm{~h} 52 \mathrm{~min}$. Thus the real travel time was overestimated by $39 \mathrm{~min}$. This represents a very good estimation. It also tells us that the CME shock did not undergo a strong acceleration/ deceleration while traveling into the interplanetary space.

\subsubsection{April 8, 2010}

The speed of the shock measured by FM method at 11.85 Rs (April 8, 07:08 UT) is $572 \mathrm{~km} \mathrm{~s}^{-1}$. With this speed the CME would require $68 \mathrm{~h} 50 \mathrm{~min}$ to arrive at the ACE spacecraft. The shock was first observed on April 11, 12:00 UT (i.e., after $76 \mathrm{~h} 52 \mathrm{~min}$ ). The real travel time was underestimated by around $8 \mathrm{~h}$. This means the CME was decelerated while traveling into the interplanetary space (the speed the CME when it arrived at ACE was $450 \mathrm{~km} \mathrm{~s}^{-1}$ ), due to the interaction with the solar wind. This effect can be mostly seen in the HI2 FOV.

\subsection{Summary}

The events observed on April 3 and 8, 2010 were well documented, and tracked from their source regions at the Sun to the moment they reached the Earth. This made them ideal candidates to be studied. The events were observed by instruments on board STEREO, SOHO, ACE, WIND, and various geomagnetic stations across the Earth. They were also isolated events with almost no influence from other dynamical events on the solar corona. This is why a large number of people analyzed these events (e.g., Liu et al. 2010, 2011; Möstl et al. 2010; Davis et al. 2011; Connors et al. 2011; Ofman \& Thompson 2011; Rouillard et al. 2011; Su et al. 2011; Seaton et al. 2011; Temmer et al. 2011; Wood et al., 2011; Xie et al. 2012). By applying different reconstruction methods, the direction of propagation and the real speeds of the events were derived. Davis et al. (2011) applied eight different methods to STEREO beacon data in order to predict the arrival time of the April 8 event at the Earth. The authors concluded that, for forecasts based on the STEREO coronagraph data, it is important to take into account the acceleration/deceleration of each $\mathrm{CME}$ as it passes through and interacts with the ambient heliosphere.

In our study we applied various reconstruction methods to coronagraph and HI data in order to derive the speeds of the two CMEs. We compared the results with similar works found in the literature, with varying degrees of concurrence. We stress on the fact that PR methods may give wrong estimates as the white-light images may be contaminated with $\mathrm{H} \alpha$ emission from prominence material.

One of the main difficulties when studying the propagation of a CME is ensuring that the same feature on the CME is tracked into interplanetary space. We have seen that it is easy to identify the shock in WL images and to fit the whole CME structure including the shock by a flux-rope-like geometrical model, which will give us the speed of the shock at distances close to the Sun. However, the shock is not easy to follow in HI images, which is the usual points where LE speeds are derived from. The LE arrival time is often compared with that of the shock at the Earth, as this is the easiest ICME feature to identify. In this way it is assumed that the shock and LE follow a similar trajectory into the interplanetary space which may not always be true. Therefore, care needs to be taken when comparing the arrival times of each feature. Even with the new speeds derived at the Sun from stereoscopic measurements, the conditions of the interplanetary medium through which the CME propagates have to be taken into account.

\section{Coronal shock propagation and solar energetic particle acceleration}

In this Section we report about SEPs and their parent solar activity. A short overview of recent results concerning the characteristics and dynamics of shocks in the corona inferred both from observations (Sect. 10.1) as well as magnetohydrodynamic simulations (Sect. 10.2) is presented. Furthermore, in Section 10.3, the implications of the shock characteristics for the production of energetic particles is discussed.

The existence of large-amplitude waves in the solar corona is a direct consequence of the dynamic magnetic activity of the corona. Indeed, shock waves launched by eruptions occurring in the corona - caused by the sudden release of energy stored in the coronal magnetic field - are believed to be intimately linked to 
the genesis of a number of transient phenomena such as metric type II bursts, Moreton and extreme ultraviolet (EUV) waves, as well as SEP events. The precise role of coronal shocks in these phenomena remains, however, a matter of lingering debate.

As a case in point, although particle acceleration at shocks driven by fast coronal mass ejections as well as magnetic reconnection in solar flares have been acknowledged as the two key processes responsible for the genesis of SEP events, the details remain under question. In the two-class paradigm of SEP events (Reames 1999), CME-driven shocks and flares are separately held responsible for generating gradual and impulsive events, respectively. Understanding in particular the multi-faceted characteristics of gradual events has, however, proved to be difficult. At energies above a few $\mathrm{MeV}$ per nucleon, gradual events show a dramatic event-to-event variability, with many events showing ion abundances and charge states generally ascribed to impulsive events (Tylka et al. 2005). Even observational evidence for a continuous, rather than bimodal, distribution of SEP event characteristics has recently been put forth, questioning the two-class classification (Cane et al. 2010).

One of the key problems in understanding the gradual SEP events is provided by the ground level enhancements (GLEs), which is SEP events extending to energies beyond $500 \mathrm{MeV}$ and, thus, observable by ground-based experiments. GLEs are relatively rare: the total number of events at present is 70 since the 1940 s, so the number of GLEs is about 10 per solar cycle. (The number of large gradual energetic particle events at lower energies, $>10 \mathrm{MeV}$, is about an order of magnitude larger.) Particle acceleration in GLEs is very rapid: Gopalswamy et al. (2011) studied the GLEs of the 23rd solar cycle and showed that the typical time from the formation of the coronal shock wave to the release of the first relativistic protons from the shock is 6 min, only. Thus, the main acceleration phase occurs while the shock is in the low corona, between about 1.4 and 2.6 solar radii (Gopalswamy et al. 2011).

An inherent difficulty in assessing our understanding of the transient phenomena is that the detailed evolution of the eruptions and the associated coronal response is not yet known from observations. Therefore, numerical models of the solar corona that are able to capture the essential features of the complex coronal large-scale dynamics are highly valuable tools for improving our understanding of eruptive phenomena.

\subsection{Observations of shocks}

A wealth of signatures indicating the presence of propagating shocks in the solar corona and interplanetary space are frequently observed at a multitude of wavelengths (see, e.g., for a review Pick et al. 2006; Vršnak \& Cliver 2008). Nevertheless, determination of the characteristics of the shock from observational data remains elusive. Recent progress has, in particular, been made in the EUV and white-light wavelength regimes.

\subsubsection{EUV shock signatures}

With the launch of the twin STEREO and the SDO spacecraft, high-cadence EUV observations from multiple vantage points have become available. With the capabilities offered by the new facilities, several observations of the initial stages of erupting CMEs with an associated EUV wave have been reported in which the CME and wave, while initially co-spatial, quickly decouple with a clear spatial offset in the lateral direction and spatial disparity between the two as a result (Patsourakos \& Vourlidas 2012). Several events have been reported in which the preceding wave is characterized by a distinct sharp dome-like wave structure that maps to the propagating on-disk EUV intensification (e.g., Veronig et al. 2010; Warmuth 2010; Ma et al. 2011; Cheng et al. 2012). While the off-limb signature of the dome quickly leaves the FOV of the instrument, the on-disk wave signature can be followed for longer times. In the June 7, 2011 event detailed by Cheng et al. (2012), the wave shows clear signs of reflection and transmission at active region boundaries and coronal holes ( $\mathrm{Li}$ et al. 2012). Furthermore, the first appearance of the distinct dome structure and the start of associated type II bursts are found to be closely related temporally (Veronig et al. 2010; Cheng et al. 2012; Gopalswamy et al. 2012).

Such observations suggest that, at least in a number of events, the EUV wave is indeed a signature of a coronal shock wave and, therefore, has the potential to yield important information of the initial characteristics of the shock. Indeed, in a few cases, the particularities of the evolution of an EUV wave associated with a SEP event have been used to understand the characteristics of the SEP event (Torsti et al. 1999; Kozarev et al. 2011). Dome-shaped shocks have been reported in MHD simulations as well (Selwa et al. 2012).

\subsubsection{White-light shock observations}

Searches for signatures of CME-driven shocks in white-light coronagraph observations have been conducted since the mid1970s following the deployment of the coronagraph on board Skylab. Despite the large number of observed CMEs, only more recently have unambiguous shock detections been reported (see Vourlidas \& Ontiveros 2009 for a review).

Observations of shocks in white-light are particularly important in that several parameters of the shock, such as the density compression ratio as well as kinematic parameters, can be inferred. Ontiveros \& Vourlidas (2009) studied $\mathrm{SOHO} / \mathrm{LASCO}$ observations of fast $\left(v>1500 \mathrm{~km} \mathrm{~s}^{-1}\right)$ CMEs that occurred in the ascending phase of solar cycle 23 (19971999). Analyzing 15 events satisfying these criteria, shock-like signatures were found in 13 of the events. In 11 of the events, the shock compression ratio could be determined, giving values ranging from 1.2 to 2.8 . Also, by fitting the observed shock shape to a three-dimensional bow shock model for three of the events, the direction of the shock could be determined. The authors found that the shock nose was within $30^{\circ}$ of the radial line from the likely source region. Recently, Kim et al. (2012) have analyzed 10 CME events that show a clear shock signature in at least three LASCO images. Calculating the density compression ratios, they have found values in the range $1.00-1.91$ with the mean being 1.18 .

Bemporad \& Mancuso (2010) present a detailed case study of the fast $\left(v \sim 1750 \mathrm{~km} \mathrm{~s}^{-1}\right)$ March 22, 2002 event observed in white light by LASCO as well as in EUV by the Ultraviolet Coronagraph Spectrometer (UVCS). Combining the data sets, the authors were able to determine the parameters of the plasma at the shock at a distance $\sim 4 R_{\odot}$ by solving the RankineHugoniot (RH) conditions for an oblique shock. The compression ratio was determined to be 2.06 , and the plasma was found to be heated across the shock from $2.3 \times 10^{5} \mathrm{~K}$ to $1.9 \times 10^{6} \mathrm{~K}$. 


\subsection{MHD modeling of shock propagation in the corona}

Magnetohydrodynamic models of the propagation of CMEs and the associated dynamics on global scales have undergone a steady improvement toward higher realism, with global three-dimensional models increasingly becoming the standard (e.g., Kleimann 2012 for a review). Most often, the MHD models consider either the coronal domain extending from the coronal base to a distance of a few tens of solar radii where the solar wind becomes super fast-magnetosonic, or the interplanetary domain from $\sim 0.1$ AU onwards.

From the viewpoint of understanding the generation of SEP events, the shock propagation in the coronal domain is of critical importance as the particles are accelerated to highest energies close to the Sun (Gopalswamy et al. 2011). Several 3D MHD studies adressing the propagation of shocks in the low corona have been conducted (e.g., Roussev et al. 2004; Manchester et al. 2005; Liu et al. 2008 for a review). A key difficulty inherent in modeling the lower corona is the question of how to realistically capture the acceleration and expansion of the solar wind. The prevailing approach of tackling this issue is by parametrizing the unknown physics involved in heating the solar corona by adopting ad hoc heating prescriptions (e.g., Cohen et al. 2008; Jacobs \& Poedts 2011; Pomoell \& Vainio 2012; and references therein). In the more advanced models of this type, the free parameters of the heating sources are related to empirical solar wind models such as the WangSheeley-Arge (Arge \& Pizzo 2000) model.

Altering the equations governing the physics naturally changes the behavior of the system, and the consequences for the dynamics of the particular problem under study must be understood. For applications involving shocks, the way in which the RH relations are modified needs to be addressed. For instance, if the polytropic index is changed as is commonly done in several models, the maximum attainable compression is likewise altered according to

$$
r_{\max }=\frac{\gamma+1}{\gamma-1} .
$$

Therefore, although the applied ad hoc heat input can represent the required heating in steady-state solar wind configurations, their applicability in cases of dynamical disturbed situations must be evaluated.

Consider a steady-state wind model using a spatially variable non-adiabatic polytropic index (designated model P1) and a model with an identical steady-state wind solution but that retains $\gamma=5 / 3$ and the RH relations of adiabatic MHD by the introduction of a time-independent energy source term (model P2), as described in detail by Pomoell \& Vainio (2012). Figure 32 shows the temperature and compression ratio at $20 \mathrm{~min}$ after the onset of a model CME for both models. The calculation is otherwise identical to that presented in Figures 2 and 3 in Pomoell \& Vainio (2012), but the flux rope is launched closer to the equator.

The plot reveals that model P1 compresses the plasma considerably more than model $\mathrm{P} 2$, which in turn heats the plasma to higher temperatures than model P1. Noteworthy is that it is not just the shock compression that is affected; also the postshock regions differ considerably both in temperature and in compression. The simulations show that, when the polytropic index is lowered, the kinetic energy of the shock is transferred increasingly into compressing the plasma rather than heating it, as expected from the RH relations. Furthermore, in the case of model P2, the $r=4$ limit for standard ideal-MHD with $\gamma=5 / 3$ is not violated.

Ignoring variations of the flow variables in the azimuthal direction in steady-state solar wind models can be argued to approximate conditions during solar minimum when the coronal plasma appears less asymmetric than during times of higher magnetic activity. The assumption of axisymmetry is clearly not valid for eruptive phenomena even during solar-minimum conditions. Therefore, depending on the application, an evaluation of the effect of the symmetry on the dynamics needs to be conducted. For the calculations discussed above, this can be done by repeating the solar wind solution in the azimuthal direction and specifying the $3 \mathrm{D}$ realization of the eruptive structure.

Figure 33 shows the result of such a 3D simulation run corresponding to the two axisymmetric cases in Figure 32. In the plotted meridional plane, the initial conditions are identical to those in the axisymmetric case. While the overall dynamics is similar, there are indeed noticeable differences. In the threedimensional setting, the shock strength is consistently smaller along the entire front. The front as a whole is more bow shock-like than the quasi-circular shape in the axisymmetric case. Both effects can be attributed to the fact that the plasma is no longer forced to deflect only in the North-South direction as in the axisymmetric case, but can also flow in the azimuthal direction. The effect of the distribution of the energy over a larger area is most clearly visible in the temperature plots for the case of an adiabatic polytropic index. In the axisymmetric case, the shock downstream is heated to temperatures beyond $10 \mathrm{MK}$ for a large area, while in the three-dimensional case only the shock nose is heated to corresponding temperatures.

The way in which the erupting Sun-encircling torus structure in the axisymmetric case is extended in the azimuthal direction in the 3D case naturally affects the solution considerably. It is conceivable that by a variation of the parameters of the model CME, a better correspondence could be achieved. In fact, an investigation along these lines has been conducted by Jacobs et al. (2007) for the case of a purely hydrodynamical model CME. A motivation for such an undertaking is the need for very high resolution of the shock structure arising, for instance, if the MHD simulation data is used as input to kinetic simulations.

\subsection{Implications for particle acceleration}

Although magnetohydrodynamic simulations naturally cannot directly address the ability of the shock to produce energetic particles, a qualitative assessment by comparing to known theoretical results is nevertheless worthwhile.

The three key parameters governing the diffusive shock acceleration process, thought to be operating in coronal shocks during SEP events, are (i) the compression ratio of the shock, (ii) the scattering mean free path around the shock, and (iii) the shock obliquity, i.e., the angle between the shock normal and the upstream magnetic field. The compression ratio determines the power-law spectral index of the accelerated particles at the shock whereas the obliquity, together with the mean free path, determines the rate of particle acceleration and, thus, the maximum energy that the process will produce in a given time. While the mean free path is not accessible through fluid modeling, the other two key parameters can be reliably obtained by MHD simulations, provided that the equation of state of the gas is realistic.

In the following, we discuss the evolution of simulated coronal shocks paying attention to compression and magnetic 

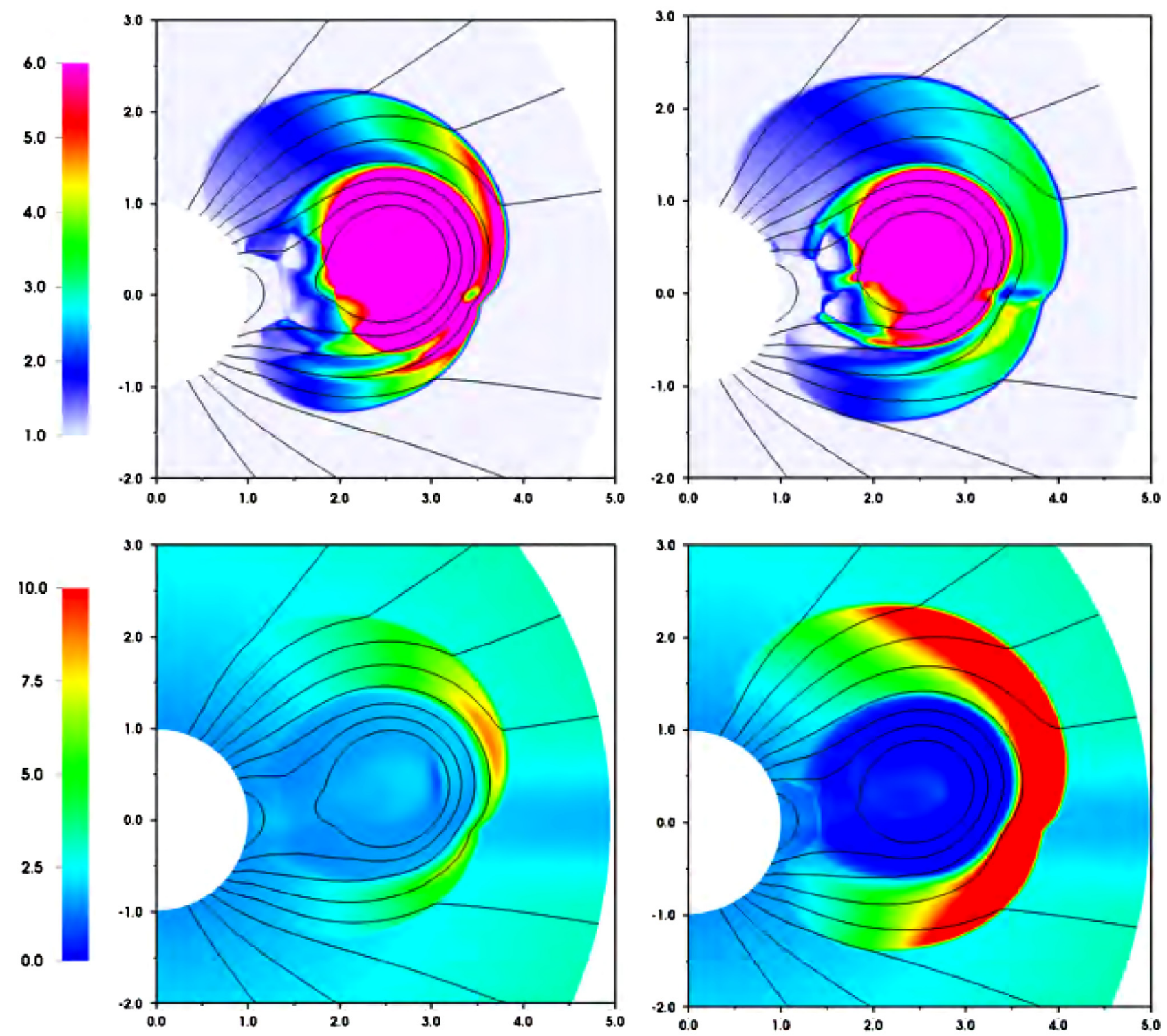

Fig. 32. Top: compression ratio $\rho(\mathbf{r}, t)=\rho(\mathbf{r}, t=0)$. Bottom: temperature in units of MK. Left images show the results for wind model P1, while the right images for P2. All images are at $t=20 \mathrm{~min}$ after onset. Note the clipping of the palette.

geometry. As a rule of thumb, the more compressive the shock is, the harder energy spectrum of accelerated particle is obtained as a result of the shock acceleration process. Increasing obliquity of the shock increases the acceleration rate, but at the same time it increases the threshold of particles to be injected into the acceleration process (Tylka \& Lee 2006). If suprathermal particles are available in the upstream medium, this injection barrier can be overcome and in that case, quasi-perpendicular shocks are more efficient in accelerating particles than quasi-parallel ones.

Figure 34 presents the evolution of the compression ratio for a CME launched $30^{\circ}$ to the North from the equatorial plane into a corona with a simple, solar-minimum-like configuration (Pomoell et al. 2011).

A common feature of the shock propagation in closed field regions is the appearance of a collapsing trap in which an upstream field line becomes connected to the shock at two points. Such a geometry has been found favorable for particle acceleration (Sandroos \& Vainio 2006), since particles are efficiently trapped in the vicinity of the shock and are forced to interact with it several times. An example of the collapsing trap configuration can be seen in Figure 34 for the outermost closed field line. The evolution of the shock obliquity for that field line is interesting: the shock starts with a quasi-parallel phase which is followed by a quasi-perpendicular phase. Performing testparticle simulations, Sandroos \& Vainio (2009a) found that in the first quasi-parallel phase, due to the lower injection threshold, the shock injects quasi-thermal particles to the acceleration process which the quasi-perpendicular phase can subsequently accelerate efficiently. In fact, in their model, Sandroos \& Vainio (2009b) found acceleration to the highest energies, extending to the GLE range, for such geometries.

The asymmetry of the eruption causes an interesting effect when comparing the potential acceleration sites on open field lines for the two hemispheres. In the northern one, the shock reaches the open field lines very quickly and remains quasi-parallel for the field lines closest to the equator. South of the current sheet, however, the shock reaches the open field lines almost an hour into the event and experiences a longer quasiperpendicular phase. Therefore, particle emission from the 

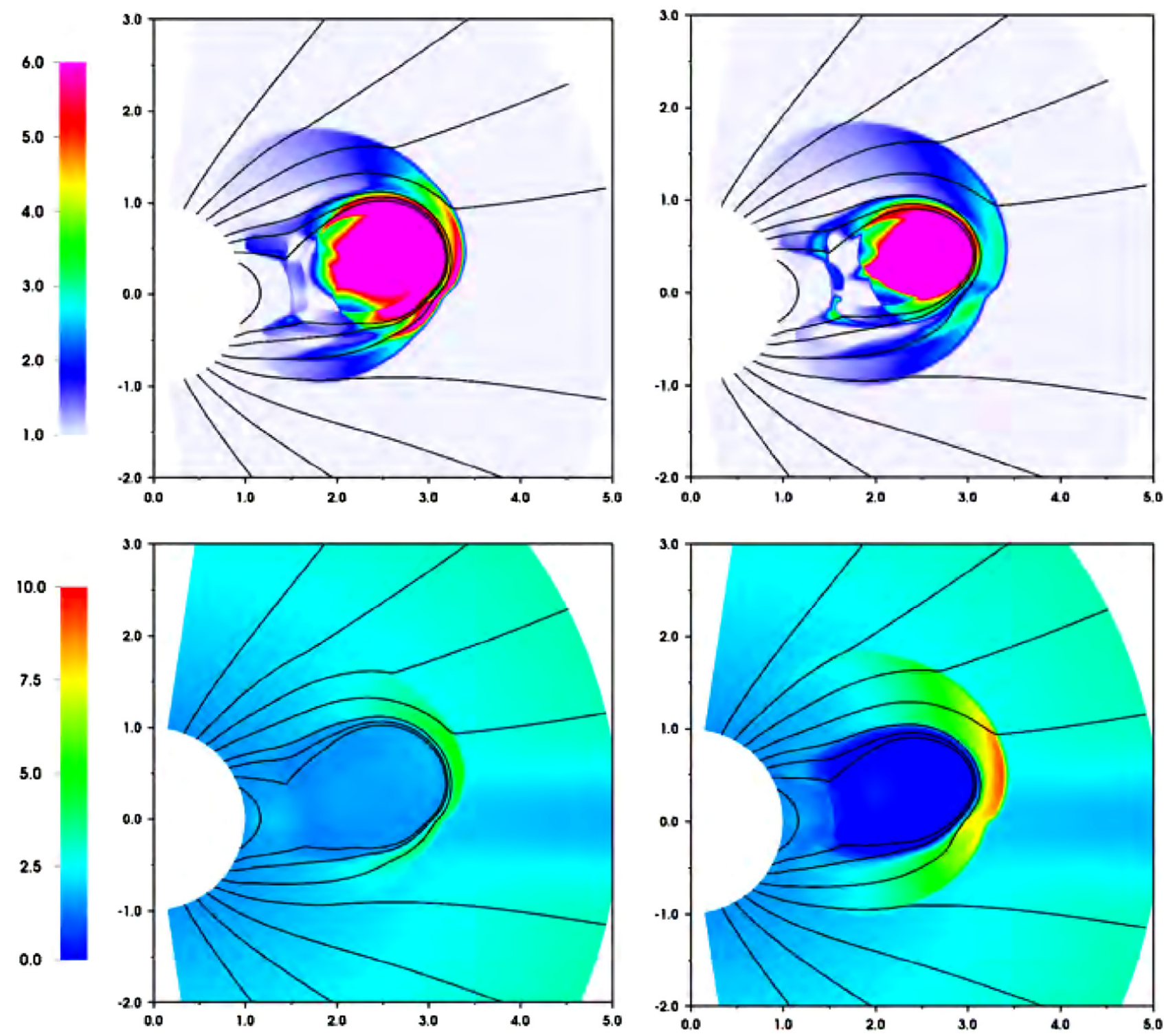

Fig. 33. Same as Figure 32 presented for the three-dimensional generalization.

south would be delayed with respect to the north, but could nonetheless produce an event extending to larger particle energies due to the more favorable geometry.

These results show that, even under relatively simple coronal conditions, a non-trivial evolution of the shock properties on coronal field lines occurs. Therefore, the evolution of parameters most crucial to particle acceleration at shocks, such as the compression ratio and obliquity, needs to be more accurately taken into account in models and simulations of particle acceleration than has been customary.

Turbulent magnetic fluctuations at kinetic scales are responsible for particle scattering around the shock. Their evolution is unaccessible to the MHD simulations. In fact, turbulence around the shock wave is most probably strongly enhanced due to streaming instabilities caused by the accelerated particles themselves (e.g., Lee 2005). Numerical models of coronal shock acceleration taking this wave generation process into account have been developed (e.g., Vainio \& Laitinen 2007; Ng \& Reames 2008; Battarbee et al. 2011). At present, however, there is no model that would combine results from an MHD simulation, providing the macroscopic evolution of the shock, with a self-consistent particle acceleration simulation that computes not only the particle distributions but also the turbulence responsible for the diffusive acceleration at the shock. This type of a model would be of key importance for Space Weather modeling and, ultimately, also for forecasting the temporal evolution of SEP events generated by CMEs.

\section{Solar irradiance variability/spectrum versus the solar activity}

As already stressed in the Introduction, one of the aims of the COST Action ES0803 concerns the Investigation of the variability of the solar radiative output, from short to long time scales.

The Spectral Solar Irradiance (SSI) is the spectrally resolved irradiance of the solar electromagnetic emissions as seen from the Earth; the TSI is its integral over the entire spectrum. Both are key quantities for the specification of the terrestrial environment and both have been the subject of many investigations (Fröhlich 2011). Direct measurements of these two quantities started in the late 1970's and it is now well established that they 

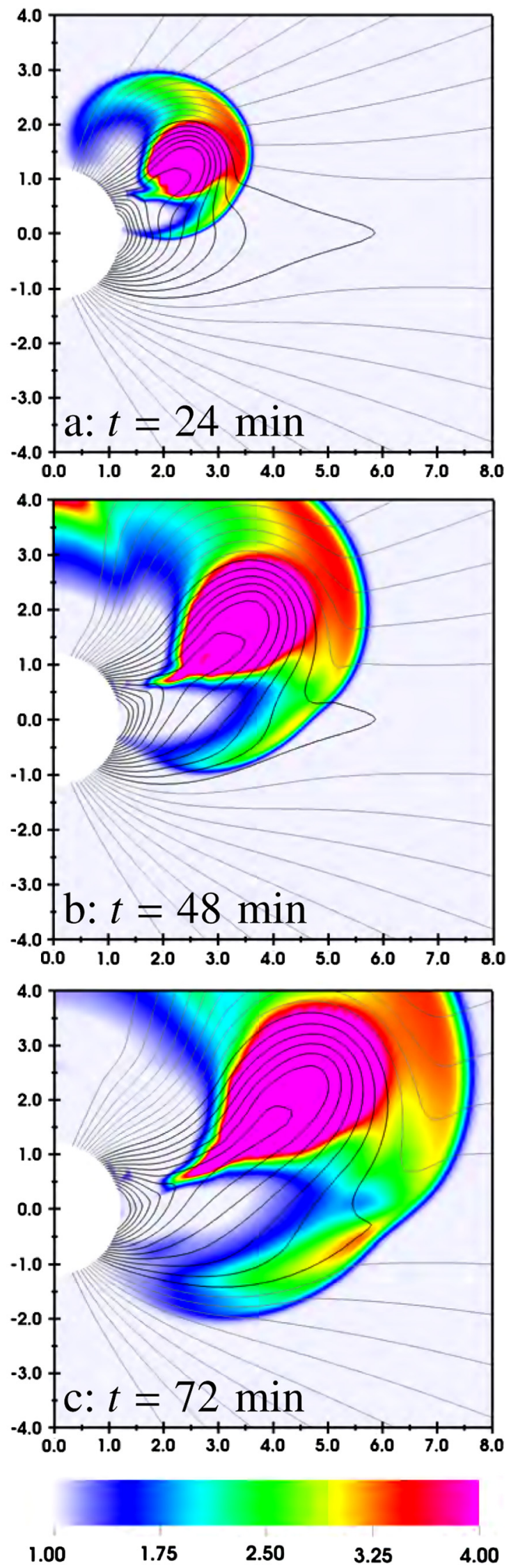

Fig. 34. Snapshots of the compression ratio at three different times after the onset of a CME. Gray curves show open field lines, while black curves show closed field lines. The colorbar is the same for all panels and has been clipped to the range $[1,4]$. undergo variations on time scales of seconds to decades, and probably much beyond.

The SSI is the main energy input to the terrestrial atmosphere and its variability is strongly wavelength dependent. The 27-day solar rotation induces a variability related to the appearance and disappearance of active regions at the solar surface. Transient events like solar flares, whose time scales range from minutes to hours, mostly affect very short wavelengths as the UV and extreme UV (XUV) bands. Finally, long-term variations, such as the 11-year solar cycle, have also an important impact on the shorter wavelengths. Figure 35 shows the time evolution of the intensity of six particular spectral lines as measured by TIMED/SEE and SORCE/SOLSTICE from early 2004 until the beginning of 2010 . Let us note that the intensities, properly normalized, reveal a common trend (the declining then the rising of the solar cycle) but also significant differences in the way the flux is modulated by the solar rotation. Although the SSI per se determines the Earth's thermal balance and climate, the effects of its variations, especially on the climate, are still a topic of controversy (Gray et al. 2010; Merkel et al. 2011).

The mechanisms by which the solar irradiance and its variability might affect the various layers of the terrestrial atmosphere are still poorly known. Part of the problem comes from the response of the atmospheric species, which is also wavelength dependent. For that reason, monitoring the SSI, creating tools for its past reconstruction, for nowcasting and for forecasting, are key issues for Space Weather and for space climate. The long-term monitoring of the SSI, however, is a major challenge. Measurements must be carried out in space, where current instruments suffer from aging, degradation as well as signal contamination. No continuous monitoring of the complete UV, visible, and Infrared (IR) spectrum was available before the launch of the SORCE satellite in 2003. This lack of observations has prompted the development of several approaches for reconstructing specific bands of the solar spectrum, especially in the UV.

The most widespread approach for reconstructing the SSI is empirical and is based on linear combinations of solar proxies. Widely used proxies are the radio flux at $10.7 \mathrm{~cm}$ (F10.7), the $\mathrm{Mg}$ II core-to-wing index, the sunspot index, and the Ca II chromospheric index (Lean et al. 2003, 2005). Many studies have shown that such proxies provide a reasonably good reconstruction of the solar variability for time scales beyond that of sporadic events. No single proxy, however, can properly reproduce the solar variability in the UV range all time scales (Dudok de Wit et al. 2009). Furthermore, the latest and deep solar minimum has shown that the correlation between these indices and different bands in the SSI is still far from being fully understood.

A second approach involves semi-empirical models that consider the solar spectrum as a linear superposition of reference spectra that originate in different regions on the solar disk (Krivova et al. 2006; Fontenla et al. 2011; Unruh et al. 2011). For a recent review, see Ermolli et al. (2012). Such regions can be determined on the basis of both solar images and magnetograms (quiet Sun, umbra, penumbra, faculae, and other disk features). A solar atmosphere model is then used to assign a spectrum to each region. This spectrum can be either determined empirically or semi-empirically using the DEM (Warren et al. 1998a, 1998b; Kretzschmar et al. 2004). Numerical codes like COSI (Shapiro et al. 2010) can also be used to determine the irradiance for different structures. Because of the lack of 


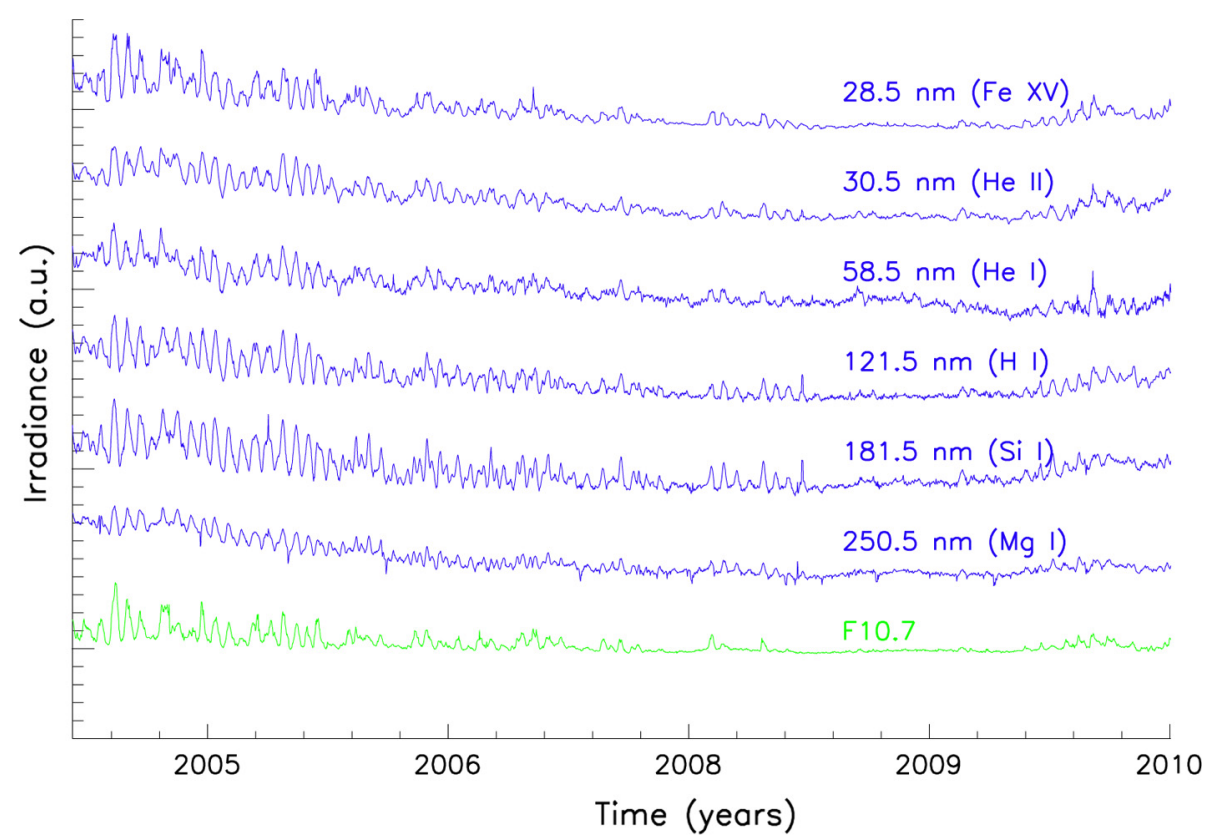

Fig. 35. Time evolution of six spectral lines and one proxy for solar activity (F10.7). All time series are properly normalized.

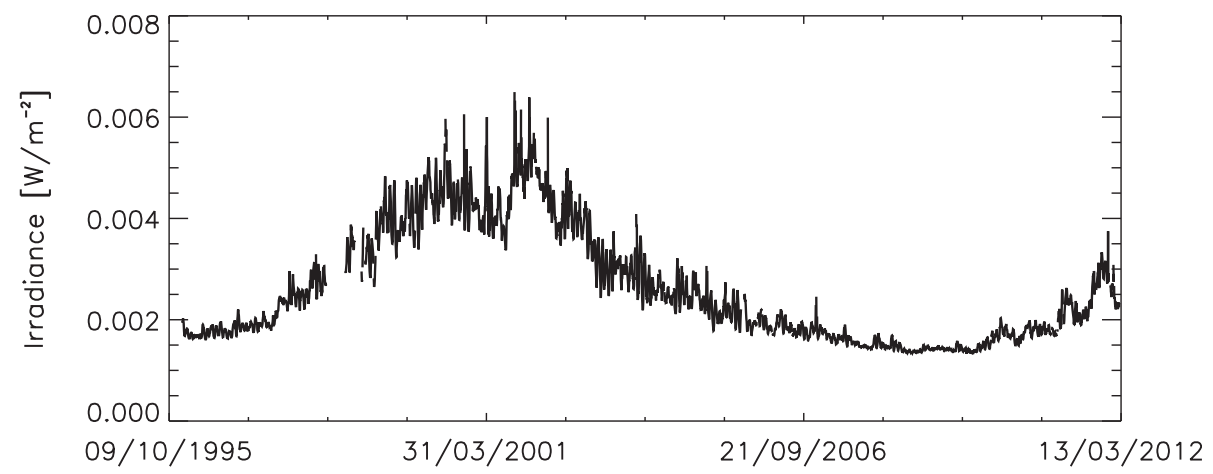

Fig. 36. Daily averaged flux around the $30.4 \mathrm{~nm}$ line from 1 January 1996 through 13 March 2012 (from SOHO/SEM).

accurate atomic data to perform the calculations of the radiative transfer in NLTE, the EUV range cannot yet be properly described by such numerical models. Most of these models cannot be used for operational purposes because they do not provide real-time spectra; very few are able to predict the spectrum. One of the few exceptions is the model by Vieira et al. (2012; http://lpc2e.cnrs-orleans.fr/:soteria/).

Significant progress has recently been made in getting a more global picture of the solar variability at different time scales and wavelengths. The primary role of sunspots and faculae in causing solar irradiance to vary has been clearly identified, but there are still unresolved questions regarding the sources of the solar variability. In particular, the recent measurements by SIM and SOLSTICE on board SORCE have revealed an unexpected behavior of the SSI in the near-UV and visible ranges (Harder et al. 2009). The variability in the near-UV is several times larger than what models give (Haigh et al. 2010). If this result is indeed confirmed, then it will significantly question our understanding of the ozone response to the solar variability and Sun-Earth connection in general (Merkel et al. 2011).

These different issues emphasize the need for making continuous SSI measurements, which questions the definition of future observation strategies. Indeed, the degradation of UV instruments seriously impacts our capacity to quantify longterm changes. Further investigations are therefore mandatory, and the idea of using radiometers instead of spectrometers is making its way. Indeed, as can be illustrated by the 16-year record of the irradiance of the He II line at $304 \AA$, measured by the SOHO/SEM instrument (see Fig. 36), relatively simple instruments with a broad spectral response can be invaluable. Radiometers are particularly suited to Space Weather needs. The recent European missions PROBA2 and PICARD, which respectively carry the LYRA (Hochedez et al. 2006), and PREMOS (Schmutz et al. 2009) radiometers, are now providing new insights into the solar variability. Both instruments cover the solar spectrum from the EUV to the near-IR. Let us also mention the SDO/EVE instrument (Woods et al. 2010) which observes the solar EUV irradiance since May 2010 with unprecedented temporal and spectral resolution.

A striking feature of the variability of the SSI is its high redundancy; this has recently been confirmed statistically for the UV range (Amblard et al. 2008). This redundancy has led to the idea of using observations of few wavelengths or spectral bands to reconstruct the solar spectrum, particularly for the UV range (Dudok de Wit et al. 2005; Kretzschmar et al. 2008; Cessateur et al. 2011). By identifying key spectral regions using either statistical method or a physical approach, a few spectral 
bands (typically 4-6) could be used to reconstruct the UV spectrum with a relative error below $20 \%$, which is definitely better than the performance of classical solar proxy models.

This new approach for reconstructing the SSI paves the way for future instrumental concepts for monitoring the solar spectrum, or even stellar spectra. Nowadays, space instruments rely on silicon ( $\mathrm{Si}$ ) photodetectors, which are technologically mature. However, present Si photodetectors for the UV suffer from serious limitations in performance and in lifetime. Recently, high-quality CBN, AlN, and diamond metal-semiconductor-metal (MSM) photodetectors have been tested for EUVUV measurements (BenMoussa et al. 2009). In the frame of solar UV irradiance monitoring, Cessateur et al. (2012) recently investigated the possibility to use such new wide-band gap materials to reconstruct the solar UV spectrum. Using different detector architectures and different materials without upstream filters (which are one of the main causes of degradation), key spectral ranges for reconstructing the variability of the spectral irradiance in the UV have been identified. The major asset of such instruments is their longer lifetime, and their small weight, which makes them particularly appropriate for Space Weather programs.

An attempt to extend this spectral reconstruction method during flares has been done with the high-cadence data of TIMED/SEE. However, the poor duty cycle (3 min of observations each $90 \mathrm{~min}$ ) did not allow us to reach conclusions. The new data of SDO/EVE will now allow us to investigate the feasibility of the reconstruction during flares. The flare irradiance model FISM (Chamberlin et al. 2008) based on the TIMED/ SEE observations is also being updated with the SDO/EVE data.

\section{Summary and conclusions}

In this paper we have reported on the research carried out in the framework of the task Sources of Solar Activity, as part of COST Action ES0803 Developing Space Weather products and services in Europe. In particular, we have reviewed the state of the art and the most recent results obtained from the investigation of phenomena that occur in the solar atmosphere and that might have consequences on the near-Earth environment, focusing on topics related to the following sub-tasks: (a) Dynamics of active regions, flares, erupting prominences, CMEs, magnetic helicity transport; (b) Simulations of solar eruptions, particle acceleration, and turbulence; (c) 3D reconstruction of CMEs from spacecraft observations.

In order to have a complete picture of these phenomena, we have initially focused on the main characteristics of ARs, describing their formation, evolution, physical properties, and decay on the basis of the most recent observations carried out by both ground-based (SST, VTT, THEMIS, IBIS/DST) and spaceborne instruments (SOHO, HINODE, SDO). We have shown how these observations, albeit at the moment characterized by different spatial resolution, have provided new clues to our understanding of the process of interaction between localized magnetic fields and plasma in the various layers of the solar atmosphere, but have on the other hand revealed that still several issues have to be further explored, in order to obtain a clear picture of the physical processes that cause the ARs formation and decay.

A paramount importance, in the framework of Space Weather, is related to the comprehension of the mechanisms causing eruptive phenomena: in this respect, a fundamental part of this review concerns the description and the techniques used to study these events, which originate from reconfiguration of magnetic fields and release of free magnetic energy stored in the solar corona. These events are also powerful accelerators of particle beams, SEPs, which can cause damage to space instruments.

We have therefore emphasized the importance of the study of the magnetic field configuration and of the helicity flux and its variations in ARs hosting eruptive events, by describing the most recent results obtained in this field.

Moreover, we have reviewed the state of the art of the knowledge of CMEs, describing the most recent results obtained by the analysis of data provided by the SOHO/ LASCO, SWAP, SDO, and STEREO instruments. The possibility to observe almost simultaneously and at different wavelengths different layers of the solar atmosphere allows us to obtain a multi-layer view of the phenomena involved and, thanks to the analysis of magnetograms, to understand many details of the magnetic nature of these phenomena.

Based on the several observations of CMEs and on their intrinsic characteristics, several CME models have been elaborated: we have reviewed the main hypotheses, characteristics, and results of these models by separating them in two categories. There are models which do not require magnetic reconnection (mass loading/off loading, kink instability, torus instability) and those where the magnetic reconnection is crucial in order to trigger and drive the CMEs (catastrophic loss of equilibrium, magnetic breakout). The main inconsistencies of these models have been briefly outlined.

The possibility to perform $3 \mathrm{D}$ reconstruction of the magnetized plasma cloud ejected from the solar atmosphere during these events allows us to have a very detailed description of the phenomena during their travel from the Sun to the Earth. This aspect has been widely illustrated by reporting some case studies, in order to evidence both the innovative reconstruction techniques and the new results obtained in this field, by which it is possible to follow the CME evolution from its source region on the Sun, to its effects on the Earth. We have also shown how these studies have given new hints to the debate on the eruptive filament - CME connection.

Some important consequences of CME occurrence, like the propagation of coronal shocks and the acceleration of SEPs have been described, together with some MHD modeling of shock propagation in the corona, stressing also the key importance of a further improvement of these models for Space Weather modeling and for forecasting the temporal evolution of SEPs events generated by CMEs.

Finally, the most recent results on the Total and Spectral Solar Irradiance have been reviewed, as these parameters play a fundamental role for the specification of the terrestrial environments. In particular, the SSI variability in the near-UV, several times larger than what is predicted by models, seems to question our understanding of the ozone response to the solar variability and Sun-Earth connection.

To conclude, we stress that important steps forward in the comprehension of phenomena originating in the Sun and that can affect the Earth environment, will be possible when new generation solar telescopes, like the EST, the ATST, IRIS, and Solar Orbiter, will be in operation because the spatial resolution achievable by these instruments will allow us to determine the details of phenomena that are currently not observable. 
Acknowledgements. This study received funding from the European Community's Seventh Framework Programme (FP7/2007/2013) under the Grant Agreement No. 218816 (SOTERIA Project, www.soteria-space.eu) and under the Grant Agreement No. 228319 (Europlanet Research Infrastructure, www.europlanet-ri.eu). It is also part of the European COST-ES0803 project. Part of this work was supported by the German Deutsche Forschungsgemeinschaft, DFG Project No. Ts $17 / 2-1$. H.C. and L.B. are members of the "Carrera del Investigador Científico" (CONICET). F.M.L. acknowledges a fellowship from CONICET. We are grateful to J. Magdalenic for the help in preparation of Figure 24. The work of M.M. was supported from the COST Action ES0803 fellowship at ROB, February to May 2010. L.R., S.P., and M. W. acknowledge support from the Belgian Federal Science Policy Office through the ESA-PRODEX program. L.R. acknowledges support from the European Union Seventh Framework Programme (FP7/2007-2013) under Grant Agreement No. 263252 COMESEP. P.R., S.L.G., and F.Z. acknowledge support from Istituto Nazionale di Astrofisica (INAF) under the PRIN-INAF 2010. F.Z. thanks the University of Catania for financial support and the International Space Science Institute (Bern) for the opportunity to discuss the results on $\mathrm{AR}$ formation at the international team meeting on "Magnetic flux emergence in the Solar Atmosphere". The research leading to these results has received funding from the European Commission's Seventh Framework Programme (FP7/2007-2013) under the Grant Agreement eHEROES (Project No. 284461). We acknowledge the use of SOHO, STEREO, Hinode, Ace, Wind, Proba2, SDO data. The SOHO data used here are produced by a consortium of the Naval Research Laboratory (USA), Max-Planck-Institut fuer Aeronomie (Germany), Laboratoire d'Astronomie (France), and the University of Birmingham (UK). SOHO is a project of international cooperation between ESA and NASA. Hinode is a Japanese mission developed and launched by ISAS/JAXA, with NAOJ as domestic partner and NASA and STFC (UK) as international partners. It is operated by these agencies in cooperation with ESA and NSC (Norway). SDO is the first mission to be launched for NASA's Living With a Star (LWS) Program. STEREO data are supplied courtesy of the Sun Earth Connection Coronal and Heliospheric Investigation (SECCHI) team. PROBA2 is a project of the Centre Spatial de Liege and the Royal Observatory of Belgium funded by the Belgian Federal Science Policy Office (BELSPO).

\section{References}

Alexander, D., Space Sci. Rev., 123, 81, 2006.

Aly, J.J., On some properties of force-free magnetic fields in infinte regions of space, Astrophys. J., 283, 349-362, 1984.

Amari, T., J.F. Luciani, J.J. Aly, and M. Tagger, Very fast opening of a three-dimensional twisted magnetic flux tube, Astrophys. J., 466, L39, 1996.

Amari, T., J.F. Luciani, Z. Mikić, and J.A. Linker, A twisted flux rope model for coronal mass ejections and two-ribbon flares, Astrophys. J., 529, L49-L52, 2000.

Amari, T., J.F. Luciani, J.J. Aly, Z. Mikić, and J.A. Linker, Coronal mass ejection: initiation, magnetic helicity, and flux ropes. I. Boundary motion-driven evolution, Astrophys. J., 585, 10731086, 2003.

Amari, T., J.J. Aly, Z. Mikic, and J. Linker, Coronal mass ejection initiation and complex topology configurations in the flux cancellation and breakout models, Astrophys. J., 671, L189L192, 2007.

Amari, T., J.-J. Aly, Z. Mikic, and J. Linker, Coronal mass ejection initiation: on the nature of the flux cancellation model, Astrophys. J., 717, L26-L30, 2010.

Amari, T., J.-J. Aly, J.-F. Luciani, Z. Mikic, and J. Linker, Coronal mass ejection initiation by converging photospheric flows: toward a realistic model, Astrophys. J., 742, L27, 2011.

Amblard, P.-O., S. Moussaoui, T. Dudok de Wit, J. Aboudarham, M. Kretzschmar, J. Lilensten, and F. Auchère, The EUV Sun as the superposition of elementary Suns, $A \& A$, 487, L13-L16, 2008.
Antiochos, S., C. DeVore, and J. Klimchuk, A model for solar coronal mass ejections, Astrophys. J., 510, 485-493, 1999.

Antiochos, S.K., J.T. Karpen, E.E. DeLuca, L. Golub, and P. Hamilton, Astrophys. J., 590, 547, 2003.

Archontis, V., F. Moreno-Insertis, K. Galsgaard, and A.W. Hood, The three-dimensional interaction between emerging magnetic flux and a large-scale coronal field: reconnection, current sheets, and jets, Astrophys. J., 635, 1299-1318, 2005.

Arge, C.N., and V.J. Pizzo, Improvement in the prediction of solar wind conditions using near-real time solar magnetic field updates, J. Geophys. Res., 105, 10465-10480, 2000.

Arregui, I., R. Oliver, and J.L. Ballester, LRSP, 9, 2, 2012.

Aulanier, G., T. Török, P. Démoulin, and E.E. DeLuca, Formation of torus-unstable flux ropes and electric currents in erupting sigmoids, Astrophys. J., 708, 314-333, 2010

Battarbee, M., T. Laitinen, and R. Vainio, Heavy-ion acceleration and self-generated waves in coronal shocks, $A \& A, \mathbf{5 3 5}, \mathrm{A} 34,2011$.

Bemporad, A., Astrophys. J., 701, 298, 2009.

Bemporad, A., Prominence 3D reconstruction in the STEREO era: a review, J. Atmos. Sol. Terr. Phys., 73 (10), 1117-1128, 2011.

Bemporad, A., and S. Mancuso, First complete determination of plasma physical parameters across a coronal mass ejection-driven shock, Astrophys. J., 720, 130-143, 2010.

Bemporad, A., F.P. Zuccarello, C. Jacobs, et al., Study of multiple coronal mass ejections at solar minimum conditions, Sol. Phys., 281, 223-236, 2012.

BenMoussa, A., A. Soltani, U. Schühle, K. Haenen, Y.M. Chong, et al., Recent developments of wide-bandgap semiconductor based UV sensors, Diamond Relat. Mater, 18(5-8), 860-864, Proceedings of Diamond 2008, the 19th European Conference on Diamond, Diamond-Like Materials, Carbon Nanotubes, Nitrides and Silicon Carbide, 2009.

Berger, M.A. and G.B. Field, The topological properties of magnetic helicity, J. Fluid Mech., 147, 133, 1984.

Berger, T.E., B. de Pontieu, L. Fletcher, et al., Sol. Phys., 190, 409, 1999.

Berger, T.E., R.A. Shine, G.L. Slater, et al., Astrophys. J., 676, L89, 2008.

Berger, T., P. Testa, A. Hillier, et al., Nature, 472, 197, 2011.

Bethge, C., C. Beck, H. Peter, and A. Lagg, $A \& A$, 537, A130, 2012.

Billings, D.E., A Guide to the Solar Corona, Academic Press, NY, London, 1966.

Bougeret, J.L., K. Goetz, M.L. Kaiser, S.D. Bale, P.J. Kellogg, M. Maksimovic, N. Monge, S.J. Monson, P.L. Astier, and S. Davy, S/ WAVES: The radio and plasma wave investigation on the STEREO mission, SSRv, 136, 487-528, 2008.

Brooks, D.H., H.P. Warren, and P.R. Young, Astrophys. J., 730, 85, 2011.

Brueckner, G.E., R.A. Howard, M.J. Koomen, C.M. Korendyke, D.J. Michels, et al., The large angle spectroscopic coronagraph (LASCO), Sol. Phys., 162, 357-402, 1995.

Burlaga, L., E. Sittler, F. Mariani, and R. Schwenn, Magnetic loop behind an interplanetary shock - Voyager, Helios, and IMP 8 observations, JGR, 86, 6673-6684, 1981.

Cane, H.V., I.G. Richardson, and T.T. von Rosenvinge, A study of solar energetic particle events of 1997-2006: their composition and associations, J. Geophys. Res., 115, A08101, 2010.

Cargill, P.J., Astrophys. J., 422, 381, 1994.

Cargill, P.J., Sol. Phys., 221, 135, 2004.

Cessateur, G., T. Dudok de Wit, M. Kretzschmar, J. Lilensten, J.-F. Hochedez, and M. Snow, Monitoring the solar UV irradiance spectrum from the observation of a few passbands, $A \& A, \mathbf{5 2 8}$, A68, 2011.

Cessateur, G., J. Lilensten, T. Dudok de Wit, A. BenMoussa, and M. Kretzschmar, New observation strategies for the solar UV spectral irradiance, J. Space Weather Space Clim., 2, A16, 2012.

Chae, J., Observational determination of the rate of magnetic helicity transport through the solar surface via the horizontal motion of the field line footpoints, Astrophys. J., 560, L95, 2001.

Chae, J., Adv. Space Res., 39, 1700, 2007. 
Chae, J., Astrophys. J., 714, 618, 2010.

Chae, J., Y. Moon, D.M. Rust, H. Wang, and P.R. Goode, Magnetic helicity pumping by twisted flux tube expansion, J. Kor. Astron. Soc., 36, 33, 2003.

Chamberlin, P.C., T.N. Woods, and F.G. Eparvier, Space Weather, 6, 5001, 2008.

Charbonneau, P., Dynamo models of the solar cycle, Living Rev. Sol. Phys., 7, 3, http://www.livingreviews.org/lrsp-2010-3, 2010.

Chen, J., Effects of toroidal forces in current loops embedded in a background plasma, Astrophys. J., 338, 453-470, 1989.

Chen, P.F., Coronal mass ejections: models and their observational basis, Living Rev. Sol. Phys., 8, 2011.

Chen, J., and J. Krall, J. Geophys. Res., 108, 1410, 2003.

Chen, P., and K. Shibata, An emerging flux trigger mechanism for coronal mass ejections, Astrophys. J., 545, 524-531, 2000.

Chen, J., R.A. Howard, G.E. Brueckner, R. Santoro, J. Krall, S.E. Paswaters, O.C. St. Cyr, R. Schwenn, P. Lamy, and G.M. Simnett, Coronal mass evidence of an erupting magnetic flux rope: LASCO coronal mass ejection of 1997 April 13, Astrophys. J., 490, L191, 1997.

Cheng, X., J. Zhang, O. Olmedo, A. Vourlidas, M.D. Ding, and Y. Liu, Investigation of the formation and separation of an extremeultraviolet wave from the expansion of a coronal mass ejection, Astrophys. J., 745, L5, 2012.

Chifor, C., H.E. Mason, D. Tripathi, H. Isobe, and A. Asai, $A \& A$, 458, 965, 2006.

Cirigliano, D., J.-C. Vial, and M. Rovira, Sol. Phys., 223, 95, 2004.

Cirtain, J.W., G. Del Zanna, E.E. DeLuca, et al., Astrophys. J., 655, 598, 2007.

Cohen, O., I.V. Sokolov, I.I. Roussev, and T.I. Gombosi, Validation of a synoptic solar wind model, J. Geophys. Res. (Space Phys.), 113, A03104, 2008

Connors, M., C.T. Russell, and V. Angelopoulos, Magnetic flux transfer in the 5 April 2010 Galaxy 15 substorm: an unprecedented observation, AnGeo, 29, 619-622, 2011.

Cremades, H., and V. Bothmer, On the three-dimensional configuration of coronal mass ejections, $A \& A, 422,307-322$, 2004.

Criscuoli, S., D. Del Moro, F. Giannattasio, et al., $A \& A$, 546, A26, 2012.

Dammasch, I.E., W. Curdt, B.N. Dwivedi, and S. Parenti, Ann. Geophys., 26, 2955, 2008.

Davis, C.J., C.A. de Koning, J.A. Davies, D. Biesecker, G. Millward, et al., A comparison of space weather analysis techniques used to predict the arrival of the Earth-directed CME and its shockwave launched on 8 April 2010, SpWea, 9, CiteID S01005, 2011.

de Koning, C.A., and V.J. Pizzo, Polarimetric localization: a new tool for calculating the CME speed and direction of propagation in near-real time, SpWea, 9, CiteID S03001, 2011.

Del Moro, D., S. Giordano, and F. Berrilli, $A \& A$, 472, 599, 2007.

Del Zanna, G., $A \& A, \mathbf{4 8 1}$, L49, 2008.

Del Zanna, G., and H.E. Mason, $A \& A$, 406, 1089, 2003.

Demoulin, P., and E. Pariat, Modelling and observations of photospheric magnetic helicity, Adv. Space Res., 43, 1013, 2009.

Dere, K.P., $A \& A, 491,561,2008$.

Dere, K.P., $A \& A, 497,287,2009$.

Dere, K.P., G.E. Brueckner, R.A. Howard, D.J. Michels, and J.P. Delaboudiniere, Astrophys. J., 516, 465, 1999.

Dere, K.P., D. Wang, and R. Howard, Three-dimensional structure of coronal mass ejections from LASCO polarization measurements, Astrophys. J., 620, L119-L122, 2005.

Ding, J.Y., and Y.Q. Hu, Astrophys. J., 674, 554, 2008.

Doschek, G.A., J.T. Mariska, H.P. Warren, et al., PASJ, 59, 707, 2007.

Dubey, G., B. van der Holst, and S. Poedts, Initiation of CMEs by magnetic flux emergence, $A \& A, 27,159-166,2006$.

Dudok de Wit, T., J. Lilensten, J. Aboudarham, P.-O. Amblard, and M. Kretzschmar, Retrieving the solar EUV spectrum from a reduced set of spectral lines, Ann. Geophys., 23, 3055-3069, 2005.
Dudok de Wit, T., M. Kretzschmar, J. Lilensten, and T. Woods, Finding the best proxies for the solar UV irradiance, Geophys. Res. Lett., 36, 10107, 2009.

Engvold, O., Sol. Phys., 70, 315, 1981.

Ermolli, I., K. Matthes, T. Dudok de Wit, et al., Atmos. Chem. Phys. Discuss., 12, 24557, 2012.

Eyles, C.J., R.A. Harrison, C.J. Davis, N.R. Waltham, B.M. Shaughnessy, et al., The heliospheric imagers onboard the STEREO mission, Sol. Phys., 254, 387-445, 2009.

Fan, Y., Magnetic fields in the solar convection zone, Living Rev. Sol. Phys., 6, 4, http://www.livingreviews.org/lrsp-2009-4, 2009.

Fan, Y., and S.E. Gibson, Numerical simulations of three-dimensional coronal magnetic fields resulting from the emergence of twisted magnetic flux tubes, Astrophys. J., 609, 1123-1133, 2004.

Fan, Y., and S.E. Gibson, Onset of coronal mass ejections due to loss of confinement of coronal flux ropes, Astrophys. J., 668, 1232$1245,2007$.

Feynman, J., and S.F. Martin, The initiation of coronal mass ejections by newly emerging magnetic flux, J. Geophys. Res., 100, 3355-3367, 1995.

Fontenla, J.M., J. Harder, W. Livingston, M. Snow, and T. Woods, High-resolution solar spectral irradiance from extreme ultraviolet to far infrared, J. Geophys. Res. (Atmos.), 116, 20108, 2011.

Forbes, T.G., J. Geophys. Res., 105, 23153, 2000.

Forbes, T., Models of coronal mass ejections and flares, edited by C.J. Schrijver, and G.L. Siscoe, Cambridge University Press, p. $159,2010$.

Forbes, T.G., and P.A. Isenberg, A catastrophe mechanism for coronal mass ejections, Astrophys. J., 373, 294-307, 1991.

Forbes, T.G., and E.R. Priest, Photospheric magnetic field evolution and eruptive flares, Astrophys. J., 446, 377, 1995.

Forbes, T.G., J.A. Linker, J. Chen, et al., CME theory and models, Space Sci. Rev., 123, 251-302, 2006.

Fröhlich, C., Total solar irradiance: what have we learned from the last three cycles and the recent minimum?, Space Sci. Rev., 366, 2011.

Galsgaard, K., V. Archontis, F. Moreno-Insertis, and A.W. Hood, The effect of the relative orientation between the coronal field and new emerging flux. I. Global properties, Astrophys. J., 666, 516$531,2007$.

Gilbert, H.R., D. Alexander, and R. Liu, Sol. Phys., 245, 287, 2007.

Gissot, S.F., J. Hochedez, P. Chainais, and J. Antoine, Sol. Phys., 252, 397, 2008.

Golub, L., E. Deluca, G. Austin, et al., Sol. Phys., 243, 63, 2007.

Gopalswamy, N., A. Lara, R.P. Lepping, et al., Geophys. Res. Lett., 27, 145,2000

Gopalswamy, N., A. Lara, S. Yashiro, M.L. Kaiser, and R.A. Howard, J. Geophys. Res., 106, 29207, 2001.

Gopalswamy, N., M. Shimojo, W. Lu, S. Yashiro, K. Shibasaki, and R.A. Howard, Astrophys. J., 586, 562, 2003.

Gopalswamy, N., H. Xie, S. Akiyama, P. Mäkelä, S. Yashiro, and I. Usoskin, Heliocentric distance of CMEs at the time of energetic particle release: revisiting the ground level enhancement events of solar cycle 23, in: Proc. Internat. Cosmic Ray Conf. 10, 157, 2011.

Gopalswamy, N., N. Nitta, S. Akiyama, P. Mäkelä, and S. Yashiro, Coronal magnetic field measurement from EUV images made by the solar dynamics observatory, Astrophys. J., 744, 72, 2012.

Goryaev, F.F., S. Parenti, A.M. Urnov, et al., $A \& A$, 523, A44, 2010.

Gosling, J.T., E. Hildner, R.M. MacQueen, et al., Sol. Phys., 48, 389 , 1976.

Gray, L.J., J. Beer, M. Geller, J.D. Haigh, M. Lockwood, et al., Solar influences on climate, Rev. Geophys., 48, RG4001, 2010.

Grechnev, V.V., A.M. Uralov, V.G. Zandanov, N.Y. Baranov, and K. Shibasaki, PASJ, 58, 69, 2006.

Guglielmino, S.L., Observational consequences of flux emergence form the photosphere to the corona: the role of interactions, 4th Hinode Science Meeting: Unsolved Problems and Recent Insights, ASP Conference series, 455, 109, 2012.

Guglielmino, S.L., and F. Zuccarello, High-resolution observations of siphon flows in a solar magnetic pore, Astrophys. J. Lett., 743, L9-L14, 2011. 
Guglielmino, S.L., L.R. Bellot Rubio, F. Zuccarello, G. Aulanier, S. Vargas Domínguez, and S. Kamio, Multiwavelength observations of small-scale reconnection events triggered by magnetic flux emergence in the solar atmosphere, Astrophys. J., 724, 10831098, 2010.

Guglielmino, S.L., V. Martínez Pillet, J.A. Bonet, J. Carlos del Toro Iniesta, L.R. Bellot Rubio, et al., The Frontier between small-scale bipoles and ephemeral regions in the solar photosphere: emergence and decay of an intermediate-scale bipole observed with SUNRISE/IMaX, Astrophys. J., 745, A160, 2012.

Gui, B., C. Shen, Y. Wang, P. Ye, J. Liu, S. Wang, and X. Zhao, Quantitative analysis of CME deflections in the corona, Sol. Phys., 271, 111-139, 2011.

Gunár, S., S. Parenti, U. Anzer, P. Heinzel, and J.-C. Vial, $A \& A$, 535, A122, 2011

Hagenaar, H.J., Ephemeral regions on a sequence of full-disk michelson doppler imager magnetograms, Astrophys. J., $\mathbf{5 5 5}$ 448-461, 2001.

Haigh, J.D., A.R. Winning, R. Toumi, and J.W. Harder, An influence of solar spectral variations on radiative forcing of climate, Nature, 467, 696-699, 2010.

Hara, H., T. Watanabe, L.K. Harra, et al., Astrophys. J., 678, L67, 2008.

Harder, J.W., J.M. Fontenla, P. Pilewskie, E.C. Richard, and T.N. Woods, Trends in solar spectral irradiance variability in the visible and infrared, Geophys. Res. Lett., 36, 7801, 2009.

Hochedez, J.-F., W. Schmutz, Y. Stockman, U. Schühle, A Benmoussa, et al., LYRA, a solar UV radiometer on Proba2, Adv. Space Res., 37, 303-312, 2006.

Holder, Z.A., R.C. Canfield, R.A. McMullen, D. Nandy, R.F. Howard, and A.A. Pevtsov, On the tilt and twist of solar active regions, Astrophys. J., 611, 1149-1155, 2004.

Howard, T., Coronal Mass Ejection: An Introduction, Springer, Berlin, 2011

Howard, R.A., J.D. Moses, A. Vourlidas, J.S. Newmark, D.G. Socker, and S.P. Plunkett, Sun Earth connection coronal and heliospheric investigation (SECCHI), Space Sci. Rev., 136, 67115,2008

Hu, Q., and B.U.O. Sonnerup, Reconstruction of magnetic clouds in the solar wind: orientations and configurations, JGRA, 107, SSH 10-1, 2002.

Hundhausen, A.J., J. Geophys. Res., 98 (A8), 177-13200, 1993.

Hundhausen, A.J., J.T. Burkepile, and O.C. St. Cyr, J. Geophys. Res., 99 (A4), 6543-6552, 1994.

Inhester, B.Stereoscopy basics for the STEREO mission, arXiv:astro$\mathrm{ph} / 0612649,2006$.

Isenberg, P.A., T.G. Forbes, and P. Demoulin, Catastrophic evolution of a force-free flux rope: a model for eruptive flares, Astrophys. $J$., 417, 368, 1993

Jacobs, C., and S. Poedts, A polytropic model for the solar wind, Adv. Space Res., 48, 1958-1966, 2011.

Jacobs, C., B. van der Holst, and S. Poedts, Comparison between 2.5D and 3D simulations of coronal mass ejections, Astrophys. J., 470, 359-365, 2007.

Joshi, V., and N. Srivastava, Bull. Astron. Soc India, 35, 447, 2007.

Joshi, A.D., and N. Srivastava, Kinematics of two eruptive prominences observed by EUVI/STEREO, Astrophys. J., 104, 730, 2011a.

Joshi, A.D., and N. Srivastava, Acceleration of coronal mass ejections from three-dimensional reconstruction of STEREO images, Astrophys. J., 739, 8, 2011b.

Kaiser, M.L., T.A. Kucera, J.M. Davila, O.C. St. Cyr, M. Guhathakurta, and E. Christian, Space Sci. Rev., 136, 5, 2008.

Kahler, S.W., and D.F. Webb, V arc interplanetary coronal mass ejections observed with the solar mass ejection imager, $J G R, \mathbf{1 1 2}$, 1, DOI:10.1029/2007JA012358, 2007.

Kilpua, E., M. Mierla, L. Rodriguez, A.N. Zhukov, N. Srivastava, and M. West, Estimating travel times of coronal mass ejections to 1 AU using multi-spacecraft coronagraph data, Sol. Phys., 279 , 477-496, 2012.
Kim, R.-S., N. Gopalswamy, Y.-J. Moon, K.-S. Cho, and S. Yashiro, Magnetic field strength in the upper solar corona using white-light shock structures surrounding coronal mass ejections, Astrophys. $J ., 746,118,2012$.

Kleimann, J., 4pi Models of CMEs and ICMEs, arXiv e-prints, 2012.

Kliem, B., and T. Török, Torus instability, Phys. Rev. Lett., 96, 255002, 2006.

Klimchuk, J.A., Theory of coronal mass ejections, in: Geophys. Monograph Series, 125, Space Weather, edited by P., Song, H.J. Singer, and G.L. Siscoe, (AGU), 143, 2001.

Klimchuk, J.A., Sol. Phys., 234, 41, 2006.

Klimchuk, J.A., S. Patsourakos, and P. J. Cargill, Astrophys. J., 682, 1351, 2008.

Ko, Y.-K., G.A. Doschek, H.P. Warren, and P.R. Young, Astrophys. J., 697, 1956, 2009.

Kozarev, K.A., K.E. Korreck, V.V. Lobzin, M.A. Weber, and N.A. Schwadron, Off-limb solar coronal wavefronts from SDO/AIA extreme-ultraviolet observations: implications for particle production, Astrophys. J., 733, L25, 2011.

Krall, J., J. Chen, and R. Santoro, Astrophys. J., 539, 964, 2000.

Kretzschmar, M., J. Lilensten, and J. Aboudarham, Variability of the EUV quiet Sun emission and reference spectrum using SUMER, $A \& A, 419,345-356,2004$

Kretzschmar, M., T. Dudok de Wit, J. Lilensten, J.-F. Hochedez, J. Aboudarham, P.-O. Amblard, F. Auchère, and S. Moussaoui, Solar EUV/FUV irradiance variations: analysis and observational strategy, Acta Geophys., 57, 42-51, 2008.

Krivova, N.A., S.K. Solanki, and L. Floyd, Reconstruction of solar UV irradiance in cycle $23, A \& A, \mathbf{4 5 2}, 631-639,2006$.

Kubo, M., T. Shimizu, and B.W. Lites, The evolution of vector magnetic fields in an emerging flux region, Astrophys. J., 595, 465-482, 2003.

Kucera, T.A., and E. Landi, Astrophys. J., 673, 611, 2008.

LaBonte, B. J., M. K. Georgoulis, and D. M. Rust, Astrophys. J., 671, 955, 2007.

Labrosse, N., P. Heinzel, J. Vial, et al., arXiv e-prints, 2010

Lean, J.L., H.P. Warren, J.T. Mariska, and J. Bishop, A new model of solar EUV irradiance variability 2. Comparisons with empirical models and observations and implications for space weather, $J$. Geophys. Res. (Space Phys.), 108, 1059, 2003.

Lean, J., G. Rottman, J. Harder, and G. Kopp, SORCE contributions to new understanding of global change and solar variability, Sol. Phys., 230, 27-53, 2005.

Lee, M.A., Coupled hydromagnetic wave excitation and ion acceleration at an evolving coronal/interplanetary shock, Astrophys. J.S, 158, 38-67, 2005.

Lepping, R.P., L.F. Burlaga, and J.A. Jones, Magnetic field structure of interplanetary magnetic clouds at $1 \mathrm{AU}, J$. Geophys. Res., 95, 11957-11965, 1990.

Li, T., J. Zhang, H. Zhao, and S. Yang, Astrophys. J., 720, 144, 2010.

Li, T., J. Zhang, H. Zhao, and S. Yang, Astrophys. J., 739 (1), 43 , 2011

Li, T., J. Zhang, S. Yang, and W. Liu, SDO/AIA observations of secondary waves generated by interaction of the 2011 June 7 global EUV wave with solar coronal structures, Astrophys. J., 746, 13, 2012.

Liewer, P.C., E.M. de Jong, J.R. Hall, R.A. Howard, W.T. Thompson, J.L. Culhane, L. Bone, and L. van Driel-Gesztelyi, Sol. Phys., 256, 57-58, 2009.

Lin, J., and T.G. Forbes, Effects of reconnection on the coronal mass ejection process, J. Geophys. Res., 105, 2375-2392, 2000.

Lin, J., T.G. Forbes, P.A. Isenberg, and P. Demoulin, The effect of curvature on flux-rope models of coronal mass ejections, Astrophys. J., 504, 1006, 1998.

Lin, Y., S.F. Martin, and O. Engvold, in: Astronomical Society of the Pacific Conference Series, Vol. 383, Subsurface and Atmospheric Influences on Solar Activity, Edited by R., Howe, R.W. Komm, K.S. Balasubramaniam, and G.J.D. Petrie, 383, 235, 2008. 
Liu, Y.C.-M., M. Opher, O. Cohen, P.C. Liewer, and T.I. Gombosi, A simulation of a coronal mass ejection propagation and shock evolution in the lower solar corona, Astrophys. J., 680, 757-763, 2008.

Liu, W., N.V. Nitta, C.J. Schrijver, A.M. Title, and T.D. Tarbell, First SDO AIA observations of a global coronal EUV "Wave": multiple components and "Ripples", Astrophys. J., 723, L53L59, 2010.

Liu, Y., J.G. Luhmann, S.D. Bale, and R.P. Lin, Solar source and heliospheric consequences of the 2010 april 3 coronal mass ejection: a comprehensive view, Astrophys. J., 734, 84, 2011.

Lopez, F.M., Extensin Espacial de CMEs Segn Imgenes Polarizadas de STEREO/COR1, Bach Thesis, Universidad Nacional de San Juan, Argentina, 104 pp, 2012.

Low, B.C., Solar activity and the corona, Sol. Phys., 167, 217-265, 1996.

Lugaz, N., A. Vourlidas, and I.I. Roussev, Deriving the radial distances of wide coronal mass ejections from elongation measurements in the heliosphere - application to CME-CME interaction, Ann. Geophys., 27, 3479-3488, 2009.

Lugaz, N., I.I. Roussev, and T.I. Gombosi, Determining CME parameters by fitting heliospheric observations: Numerical investigation of the accuracy of the methods, Adv. Space Res., 48, 292 299, 2011.

Lynch, B.J., S.K. Antiochos, C.R. DeVore, J.G. Luhmann, and T.H. Zurbuchen, Topological evolution of a fast magnetic breakout CME in three dimensions, Astrophys. J., 683, 11921206, 2008.

Ma, S., J.C. Raymond, L. Golub, J. Lin, H. Chen, P. Grigis, P. Testa, and D. Long, Observations and interpretation of a low coronal shock wave observed in the EUV by the SDO/AIA, Astrophys. $J$., 738, 160, 2011.

MacNeice, P., S.K. Antiochos, A. Phillips, et al., A numerical study of the breakout model for coronal mass ejection initiation, Astrophys. J., 614, 1028-1041, 2005.

MacQueen, R.M., and R.R. Fisher, Sol. Phys., 89, 89, 1983.

MacTaggart, D., Flux emergence within mature solar active regions, $A \& A, \mathbf{5 3 1}, \mathrm{A} 108,2011$.

Manchester, W.B.IV, T.I. Gombosi, D.L. De Zeeuw, I.V. Sokolov, I.I. Roussev, K.G. Powell, J. Kóta, G. Tóth, and T.H. Zurbuchen, Coronal mass ejection shock and sheath structures relevant to particle acceleration, Astrophys. J., 622, 1225-1239, 2005.

Manoharan, P.K., and A. Mujiber Rahman, J. Atmos. Sol. Terr. Phys., 73, 671, 2011.

Martens, P.C.H., C.C. Kankelborg, and T.E. Berger, Astrophys. J., 537, 471, 2000.

Martin, S.F., Sol. Phys., 182, 107, 1998

Martin, S.F., Adv. Space Res., 32, 1883, 2003.

Martínez González, M.J., and L.R. Bellot Rubio, Emergence of small-scale magnetic loops through the quiet solar atmosphere, Astrophys. J., 700, 1391-1403, 2009.

Merkel, A.W., J.W. Harder, D.R. Marsh, A.K. Smith, J.M. Fontenla, and T.N. Woods, The impact of solar spectral irradiance variability on middle atmospheric ozone, Geophys. Res. Lett., 38, 13802, 2011.

Messerotti, M., F. Zuccarello, S.L. Guglielmino, V. Bothmer, J. Lilensten, G. Noci, M. Storini, and H. Lundstedt, Space Sci. Rev., 147, 121, 2009.

Mierla, M., J. Davila, W. Thompson, B. Inhester, N. Srivastava, M. Kramar, O.C. StCyr, G. Stenborg, and R.A. Howard, A quick method for estimating the propagation direction of coronal mass ejections using STEREO-COR1 images, Sol. Phys., 252, 385396, 2008.

Mierla, M., B. Inhester, C. Marque, L. Rodriguez, S. Gissot, A. Zhukov, D. Berghmans, and J. Davila, On 3D reconstruction of coronal mass ejections: I. Method description and application to SECCHI-COR data, Sol. Phys., 259, 123-141, 2009.

Mierla, M., B. Inhester, A. Antunes, Y. Boursier, J.P. Byrne, et al., On the 3-D reconstruction of coronal mass ejections using coronagraph data, Ann. Geophys., 28, 203-215, 2010.
Mierla, M., I. Chifu, B. Inhester, L. Rodriguez, and A. Zhukov, Low polarised emission from the core of coronal mass ejections, $A \& A$, 530, L1-L4, DOI: 10.1051/0004-6361/201016295, 2011.

Mikic, Z., and J.A. Linker, Disruption of coronal magnetic field arcades, Astrophys. J., 430, 898-912, 1994.

Moon, Y.-J., G.S. Choe, H. Wang, et al., Astrophys. J., 581, 694 , 2002.

Moran, T.G., and J.M. Davila, Three-dimensional polarimetric imaging of coronal mass ejections, Science, 305, 66-70, 2004.

Moreno-Insertis, F., K. Galsgaard, and I. Ugarte-Urra, Hinode Observations and Three-dimensional Computer Modeling, Astrophys. J., 673, L211-L214, 2008.

Möstl, C., M. Temmer, T. Rollett, C.J. Farrugia, Y. Liu, A.M. Veronig, M. Leitner, and H.K. Biernat, STEREO and Wind observations of a fast ICME flank triggering a prolonged geomagnetic storm on 5-7 April 2010, GeoRL, 37, L24103, 2010.

$\mathrm{Ng}$, C.K., and D.V. Reames, Shock acceleration of solar energetic protons: the first 10 minutes, Astrophys. J., 686, L123-L126, 2008.

November, L.J., and G.W. Simon, Precise proper-motion measurement of solar granulation, Astrophys. J., 333, 427, 1988.

Ofman, L., and B.J. Thompson, SDO/AIA observation of KelvinHelmholtz instability in the solar corona, Astrophys. J. Lett., 734, id L11, 2011.

Oliver, R., Space Sci. Rev., 39, 2009.

Ontiveros, V., and A. Vourlidas, Quantitative measurements of coronal mass ejection-driven shocks from LASCO observations, Astrophys. J., 693, 267-275, 2009.

Panasenco, O., S.F. Martin, A.D. Joshi, and N. Srivastava, Rolling motion in erupting prominences observed by STEREO, J. Atmos. Sol. Terr. Phys., 73, 1129-1137, 2011.

Parenti, S., and J.-C. Vial, $A \& A, \mathbf{4 6 9}, 1109,2007$.

Parenti, S., and P.R. Young, $A \& A$, 492, 857, 2008

Parenti, S., J.-C. Vial, and P. Lemaire, Sol. Phys., 220, 61, 2004

Parenti, S., P. Lemaire, and J Vial, $A \& A, 443,685,2005$ a.

Parenti, S., J.-C. Vial, and P. Lemaire, $A \& A, 443,679,2005 \mathrm{~b}$.

Parenti, S., E. Buchlin, P.J. Cargill, S. Galtier, and J.-C. Vial, 651, 1219,2006

Parenti, S., F. Reale, and K.K. Reeves, $A \& A$, 517, A41, 2010.

Parenti, S., B. Schmieder, P. Heinzel, and L. Golub, On the nature of prominence emission observed by SDO/AIA, Astrophys. J., 754, A66, 2012

Pariat, E., P. Démoulin, and M.A. Berger, Photospheric flux density of magnetic helicity, $A \& A, \mathbf{4 3 9}, 1191,2005$.

Park, S.H., J. Lee, G.S. Choe, J. Chae, H. Jeong, G. Yang, J. Jing, and H. Wang, 686, 1379, 2008.

Park, S.H., J. Chae, and H. Wang, Astrophys. J., 718, 43, 2010.

Patsourakos, S., and A. Vourlidas, On the nature and genesis of EUV Waves: a synthesis of observations from SOHO, STEREO, SDO, and hinode, arXiv e-prints, http://arxiv.org/abs/1203.1135, 2012.

Pick, M., T.G. Forbes, G. Mann, H.V. Cane, J. Chen, et al., Multiwavelength observations of CMEs and associated phenomena. Report of Working Group F, Space Sci. Rev., 123, 341-382, 2006.

Pomoell, J., and R. Vainio, Influence of solar wind heating formulations on the properties of shocks in the corona, Astrophys. J., 745, 151, 2012

Pomoell, J., R. Vainio, and R. Kissmann, MHD simulation of the evolution of shock structures in the solar corona: implications for coronal shock acceleration, Astrophys. Space Sci. Trans., 7, 387394, 2011.

Porter, L.J., and J.A. Klimchuk, Astrophys. J., 454, 499, 1995.

Priest, E.R., Astrophys. J., 328, 848, 1988.

Raadu, M.A., J.M. Malherbe, B. Schmieder, and P. Mein, Sol. Phys., 109, 59, 1987

Rachmeler, L.A., C.E. DeForest, and C.C. Kankelborg, Reconnectionless CME eruption: putting the aly-sturrock conjecture to rest 2009, Astrophys. J., 693, 1431, 2009.

Reale, F., Living Rev. Sol. Phys., 7, 5, 2010.

Reale, F., S. Parenti, K.K. Reeves, et al., Science, 318, 1582, 2007.

Reale, F., P. Testa, J.A. Klimchuk, and S. Parenti, Astrophys. J., 698, $756,2009$. 
Reames, D.V., Particle acceleration at the Sun and in the heliosphere, Space Sci. Rev., 90, 413-491, 1999.

Robbrecht, E., and D. Berghmans, Automated recognition of coronal mass ejections (CMEs) in near-real-time data, $A \& A, \mathbf{4 2 5}, 1097-$ 1106, 2004.

Robbrecht, E., D. Berghmans, and R.A.M. Van der Linden, Automated LASCO CME catalog for solar cycle 23: are CMEs scale invariant? Astrophys. J., 691, 1222-1234, 2009.

Romano, P., and F. Zuccarello, Flare occurrence and the spatial distribution of the magnetic helicity flux, $A \& A, \mathbf{5 3 5}, \mathrm{A} 1,2012$.

Romano, P., E. Pariat, M. Sicari, and F. Zuccarello, A solar eruption triggered by the interaction between two magnetic flux systems with opposite magnetic helicity, $A \& A, \mathbf{5 2 5}, \mathrm{A} 13,2011$.

Romano, P., F. Zuccarello, S. Poedts, A. Soenen, and F.P. Zuccarello, Magnetic helicity and active filament configuration, $A \& A, \mathbf{5 0 6}$, 895, 2009

Rouillard, A.P., D. Odstrcil, N.R. Sheeley, A. Tylka, A. Vourlidas, et al., Interpreting the properties of solar energetic particle events by using combined imaging and modeling of interplanetary shocks, Astrophys. J., 735, ID 7, 2011.

Roussev, I.I., T.G. Forbes, T.I. Gombosi, et al., A three-dimensional flux rope model for coronal mass ejections based on a loss of equilibrium, Astrophys. J., 588, L45-L48, 2003.

Roussev, I.I., I.V. Sokolov, T.G. Forbes, T.I. Gombosi, M.A. Lee, and J.I. Sakai, A numerical model of a coronal mass ejection: shock development with implications for the acceleration of $\mathrm{GeV}$ protons, Astrophys. J., 605, L73-L76, 2004.

Sainz Dalda, A., S. Vargas Domínguez, and T.D. Tarbell, Magnetic topology of a naked sunspot: is it really naked? Astrophys. J., 746, L13, 2012.

Sandroos, A., and R. Vainio, Particle acceleration at shocks propagating in inhomogeneous magnetic fields, $A \& A, 455,685$ 695, 2006.

Sandroos, A., and R. Vainio, Reacceleration of flare ions in coronal and interplanetary shock waves, Astrophys. J. Suppl., 181, 183196, 2009a.

Sandroos, A., and R. Vainio, Diffusive shock acceleration to relativistic energies in the solar corona, $A \& A, \mathbf{5 0 7}, \mathrm{L} 21-\mathrm{L} 24$, 2009b.

Schmieder, B., M.A. Raadu, and J.E. Wiik, $A \& A$, 252, 353, 1991.

Schmieder, B., C. Delannée, D.Y. Yong, J.C. Vial, and M. Madjarska, $A \& A, \mathbf{3 5 8}, 728,2000$.

Schmutz, W., A. Fehlmann, G. Hüsen, P. Meindl, R. Winkler, et al., The PREMOS/PICARD instrument calibration, Metrologia, 46, S202-S206, 2009.

Schrijver, C.J., A.W. Sandman, M.J. Aschwanden, and M.L. De Rosa, Astrophys. J., 615, 512, 2004

Schrijver, C.J., C. Elmore, B. Kliem, T. Török, and A.M. Title, Astrophys. J., 674, 586-595, 2008.

Schuck, P.W., Local correlation tracking and the magnetic induction equation, Astrophys. J., 632, L53, 2005.

Seaton, D.B., M. Mierla, D. Berghmans, A.N. Zhukov, and L. Dolla, Astrophys. J., 727, L10, 2011.

Selwa, M., S. Poedts, and C.R. DeVore, Dome-shaped EUV waves from rotating active regions, Astrophys. J., 747, L21, 2012.

Shapiro, A.I., W. Schmutz, M. Schoell, M. Haberreiter, and E. Rozanov, NLTE solar irradiance modeling with the COSI code, $A \& A, \mathbf{5 1 7}, \mathrm{A} 48,2010$.

Sheeley, N.R., J.H. Walters, Y.-M. Wang, and R.A. Howard, Continuous tracking of coronal outflows: two kinds of coronal mass ejections, J. Geophys. Res., 104, 24739-24768, 1999.

Smyrli, A., F. Zuccarello, P. Romano, F.P. Zuccarello, S.L. Guglielmino, D. Spadaro, A.W. Hood, and D. Mackay, Trend of photospheric magnetic helicity flux in active regions generating halo coronal mass ejections, $A \& A, \mathbf{5 2 1}, \mathrm{A} 56,2010$.

Solanki, S.K., Sunspots: an overview, A\&A Review, 11, 153-286, 2003

Spadaro, D., S. Billotta, L. Contarino, P. Romano, and F. Zuccarello, AFS dynamic evolution during the emergence of an active region, $A \& A, \mathbf{4 2 5}, 309-319,2004$.
Srivastava, N., and A. Ambastha, Ap\&SS, 262, 29, 1998.

Srivastava, N., A. Ambastha, and A. Bhatnagar, Sol. Phys., 133, 339, 1991.

Srivastava, N., R. Schwenn, B. Inhester, G. Stenborg, and B. Podlipnik, Space Sci. Rev., 87, 303, 1999.

Srivastava, N., R. Schwenn, B. Inhester, S.F. Martin, and Y. Hanaoka, Astrophys. J., 534, 468, 2000.

Sterling, A.C., and R.L. Moore, Astrophys. J., 602, 1024, 2004a.

Sterling, A.C., and R.L. Moore, Astrophys. J., 613, 1221, 2004b.

Sterling, A.C., and R.L. Moore, Astrophys. J., 630, 1148, 2005.

Sterling, A.C., L.K. Harra, and R.L. Moore, Astrophys. J., 669, 1359, 2007.

Sturrock, P.A., Maximum energy of semi-infinite magnetic field configurations, Astrophys. J., 380, 655-659, 1991.

$\mathrm{Su}$, Y., V. Surges, A. van Ballegooijen, E. DeLuca, and L. Golub, Observations and magnetic field modeling of the flare/coronal mass ejection event on 2010 April 8, Astrophys. J., 734, id 53, 2011.

Tandberg-Hanssen, E., S.F. Martin, and R.T. Hansen, Sol. Phys., 65, $357,1980$.

Temmer, M., A.M. Veronig, E.P. Kontar, S. Krucker, and B. Vršnak, Astrophys. J., 712, 1410, 2010.

Temmer, M., T. Rollett, C. Mostl, A. Veronig, B. Vršnak Bojan, and D. Odstrcil, Influence of the ambient solar wind flow on the propagation behavior of interplanetary coronal mass ejections, Astrophys. J., 743, CiteID 101, 2011.

Teriaca, L., D. Banerjee, and J.G. Doyle, $A \& A, \mathbf{3 4 9}, 636,1999$.

Teriaca, L., D. Banerjee, A. Falchi, J.G. Doyle, and M.S. Madjarska, $A \& A, \mathbf{4 2 7}, 1065,2004$

Thernisien, A., R.A. Howard, and A. Vourlidas, Modeling of flux rope coronal mass ejections, Astrophys. J., 652, 763-773, 2006.

Thernisien, A., A. Vourlidas, and R.A. Howard, Forward modelling of coronal mass ejections using stereo-secchi data, Sol. Phys., 256, 1110, 2009.

Thernisien, A., A. Vourlidas, and R.A. Howard, CME reconstruction: pre-STEREO and STEREO era, JASTP, 73, 1156-1165, 2011.

Thompson, W.T., Strong rotation of an erupting quiescent polar crown prominence, JASTP, 73, 1138, 171147, 2011.

Tian, L., D. Alexander, Y. Liu, and J. Yang, Magnetic twist and writhe of $\delta$ active regions, Sol. Phys., 229, 63-77, 2005.

Titov, V.S., and P. Démoulin, Basic topology of twisted magnetic configurations in solar flares, $A \& A, \mathbf{3 5 1}, 707-720,1999$.

Tripathi, D., H. Isobe, and H.E. Mason, $A \& A$, 453, 1111, 2006

Tripathi, D., H.E. Mason, G. Del Zanna, and P.R. Young, $A \& A, \mathbf{5 1 8}$ A42, 2010

Tripathi, D., J.A. Klimchuk, and H.E. Mason, Astrophys. J., $\mathbf{7 4 0}$ $111,2011$.

Torsti, J., L.G. Kocharov, M. Teittinen, and B.J. Thompson, Injection of $\gtrsim 10 \mathrm{MeV}$ protons in association with a coronal moreton wave, Astrophys. J., 510, 460-465, 1999.

Tylka, A.J., and M.A. Lee, A model for spectral and compositional variability at high energies in large, gradual solar particle events, Astrophys. J., 646, 1319-1334, 2006.

Tylka, A.J., C.M.S. Cohen, W.F. Dietrich, M.A. Lee, C.G. Maclennan, R.A. Mewaldt, C.K. Ng, and D.V. Reames, Shock geometry, seed populations, and the origin of variable elemental composition at high energies in large gradual solar particle events, Astrophys. J., 625, 474-495, 2005.

Török, T., and B. Kliem, The evolution of twisting coronal magnetic flux tubes, $A \& A$, 406, 1043-1059, 2003.

Török, T., and B. Kliem, Numerical simulations of fast and slow coronal mass ejections, Astron. Nachr., 328, 743, 2007.

Török, T., and B. Kliem, Confined and ejective eruptions of kinkunstable flux ropes, Astrophys. J., 630, L97-L100, 2005.

Török, T., B. Kliem, and V.S. Titov, Ideal kink instability of a magnetic loop equilibrium, $A \& A$, 413, L27-L30, 2004.

Ugarte-Urra, I., H.P. Warren, and D.H. Brooks, Astrophys. J., 695, $642,2009$.

Ugarte-Urra, I., A.R. Winebarger, and H.P. Warren, Astrophys. J., 643, 1245, 2006. 
Unruh, Y.C., W.T. Ball, and N.A. Krivova, Solar irradiance models and measurements: a comparison in the $220 \mathrm{~nm}$ to $240 \mathrm{~nm}$ wavelength band, ArXiv e-prints, 2011.

Vaiana, G.S., and R. Rosner, $A R A \& A, 16,393,1978$.

Vainio, R., and T. Laitinen, Monte Carlo simulations of coronal diffusive shock acceleration in self-generated turbulence, Astrophys. J., 658, 622-630, 2007.

van Ballegooijen, A.A., and P.C.H. Martens, Formation and eruption of solar prominences, Astrophys. J., 343, 971-984, 1989.

van der Holst, B., W. Manchester, I.V. Sokolov, et al., Breakout coronal mass ejection or streamer blowout: the bugle effect, Astrophys. J., 693, 1178-1187, 2009.

van Driel-Gesztelyi, L., Emergence and loss of magnetic flux on the solar surface, in SOLMAG 2002, Proceedings of the Magnetic Coupling of the Solar Atmosphere Euroconference, edited by H., Sawaya-Lacoste 505, Noordwijk, Netherlands, ESA Publications Division, 113-120, 2002.

Veronig, A.M., N. Muhr, I.W. Kienreich, M. Temmer, and B. Vršnak, First observations of a dome-shaped large-scale coronal extreme-ultraviolet wave, Astrophys. J., 716, L57-L62, 2010.

Vial, J.-C., K. Olivier, A.A. Philippon, A. Vourlidas, and V. Yurchyshyn, $A \& A$, 541, A108, 2012.

Viall, N.M., and J.A. Klimchuk, Astrophys. J., 738, 24, 2011.

Vieira, L.E., T. Dudok de Wit, and M. Kretzschmar, Short-term forecast of the total and spectral solar irradiance, submitted, 2012.

Vourlidas, A., and Russell A. Howard, The proper treatment of coronal mass ejection brightness: a new methodology and implications for observations, Astrophys. J., 642, 1216-1221, 2006.

Vourlidas, A., and V. Ontiveros, X., Ao, and G.Z.R. Burrows, A review of coronagraphic observations of shocks driven by coronal mass ejections, Am. Inst. Phys. Conf. Ser., 1183, 139-146, 2009.

Vourlidas, A., B. Sanchez Andrade-Nuño, E. Landi, S. Patsourakos, L. Teriaca, U. Schühle, C.M. Korendyke, and I. Nestoras, SoPh, 261, 53, 2010.

Vršnak, B., Deceleration of coronal mass ejections, Sol. Phys., 202, 173-189, 2001.

Vršnak, B., and E.W. Cliver, Origin of coronal shock waves. Invited review, Sol. Phys., 253, 215-235, 2008.

Vršnak, B., V. Ruždjak, B. Rompolt, Sol. Phys., 136, 151, 1991.

Vršnak, B., V. Ruždjak, B. Rompolt, D. Roša, and P. Zlobec, Sol. Phys., 146, 147, 1993.

Vršnak, B., T. Žic, T.V. Falkenberg, et al., $A \& A, \mathbf{5 1 2}, \mathrm{A} 43,2010$.

Warmuth, A., Large-scale waves in the solar corona, Adv. Space Res., 45, 527-536, 2010.

Warren, H.P., and A.R. Winebarger, Astrophys. J., 666, 1245, 2007.

Warren, H.P., J.T. Mariska, and J. Lean, A new reference spectrum for the EUV irradiance of the quiet Sun 1. Emission measure formulation, J. Geophys. Res., 103, 12077-12090, 1998a.

Warren, H.P., J.T. Mariska, and J. Lean, A new reference spectrum for the EUV irradiance of the quiet Sun 2, Comparisons with observations and previous models, 103, 12091-12102, 1998b.

Warren, H.P., I. Ugarte-Urra, G.A. Doschek, D.H. Brooks, and D.R. Williams, Astrophys. J., 686, L131, 2008.

Warren, H.P., D.H. Brooks, and A.R. Winebarger, Astrophys. J., 734, 90, 2011.

Webb, D.F., and T.A. Howard, LRSP, 9, 3, 2012.

Weber, M.A., J.T. Schmelz, E.E. DeLuca, and J.K. Roames, Astrophys. J., 635, L101, 2005.

Winebarger, A.R., and H.P. Warren, Astrophys. J., 626, 543, 2005.

Cite this article as: Zuccarello F, Balmaceda L, Cessateur G, Cremades H, Guglielmino S, et al.: Solar activity and its evolution across the corona: recent advances. J. Space Weather Space Clim., 2013, 3, A18.
Winebarger, A.R., J.T. Schmelz, H.P. Warren, S.H. Saar, and V.L. Kashyap, Astrophys. J., 740, 2, 2011.

Wolfson, R., and B. Dlamini, Cross-field currents: an energy source for coronal mass ejections? Astrophys. J., 483, 961, 1997.

Wood, B.E., C.-C. Wu, R.A. Howard, D.G. Socker, and A.P. Rouillard, Empirical reconstruction and numerical modeling of the first geoeffective coronal mass ejection of solar cycle 24 , Astrophys. J., 729, ID 70, 2011.

Woods, T.N., F.G. Eparvier, R. Hock, A.R. Jones, D. Woodraska, et al., Extreme ultraviolet variability experiment (EVE) on the solar dynamics observatory (SDO): overview of science objectives, Instrument Design, Data Products, and Model Developments, Jan, 3, 2010.

Xie, H., D. Odstrcil, L. Mays, O.C. St. Cyr, N. Gopalswamy, and H. Cremades, Understanding shock dynamics in the inner heliosphere with modeling and Type II radio data: The 2010-04-03 event, J. Geophys. Res. A: Space Phys., 117, id A04105, 2012.

Yashiro, S., N. Gopalswamy, G. Michalek, et al., J. Geophys. Res., 109, 7105, 2004.

Yokoyama, T., and K. Shibata, Magnetic reconnection as the origin of X-ray jets and $\mathrm{H} \alpha$ surges on the Sun, Nature, 375, 42-44, 1995.

Yurchyshyn, V., Relationship between EIT posteruption arcades, coronal mass ejections, the coronal neutral line, and magnetic clouds, Astrophys. J., 675, L49-L52, 2008.

Zhang, J., and K.P. Dere, Astrophys. J., 649, 1100, 2006.

Zuccarello, F., V. Battiato, L. Contarino, P. Romano, D. Spadaro, and L. Vlahos, AFS dynamics in a short-lived active region, $A \& A$, 442, 661-671, 2005.

Zuccarello, F., V. Battiato, L. Contarino, S. Guglielmino, P. Romano, and D. Spadaro, A C-level flare observed in an arch filament system: reconnection between pre-existing and emerging field lines? $A \& A, 488,1117-1123,2008$.

Zuccarello, F.P., A. Soenen, S. Poedts, F. Zuccarello, and C. Jacobs, Initiation of coronal mass ejections by magnetic flux emergence in the framework of the breakout model, Astrophys. J., 689, L157L160, 2008.

Zuccarello, F., P. Romano, S.L. Guglielmino, M. Centrone, S. Criscuoli, I. Ermolli, F. Berrilli, and D. Del Moro, Observation of bipolar moving magnetic features streaming out from a naked spot, $A \& A, \mathbf{5 0 0}, \mathrm{L} 5-\mathrm{L} 8,2009 \mathrm{a}$.

Zuccarello, F., S.L. Guglielmino, V. Battiato, L. Contarino, D. Spadaro, and P. Romano, Emergence and evolution of active and ephemeral regions: comparison between observations and models, Acta Geophys., 57, 15-23, 2009b.

Zuccarello, F.P., C. Jacobs, A. Soenen, et al., Modelling the initiation of coronal mass ejections: magnetic flux emergence versus shearing motions, $A \& A, \mathbf{5 0 7}, 441-452,2009$.

Zuccarello, F.P., P. Romano, F. Zuccarello, and S. Poedts, Magnetic helicity balance during a filament eruption that occurred in active region NOAA 9682, $A \& A, \mathbf{5 3 0}, 36-530,2011$.

Zuccarello, F.P., A. Bemporad, C. Jacobs, et al., Astrophys. J., 744, $66,2012 \mathrm{a}$.

Zuccarello, F.P., Z. Meliani, and S. Poedts, Numerical modeling of the initiation of coronal mass ejections in active region NOAA 9415, Astrophys. J., 758, 117, 2012b.

Zuccarello, F.P., P. Romano, F. Zuccarello, and S. Poedts, The role of photospheric shearing motions in a filament eruption related to the 2010 April 3 coronal mass ejection, $A \& A, 537$, A28, 2012c. 